\title{
Role of Histone Metabolism and Chromatin Structure in DNA Repair
}

\author{
Dissertation \\ for the award of the degree \\ "Doctor of Philosophy" \\ Division of Mathematics and Natural Sciences \\ of the Georg-August-Universität Göttingen
}

Submitted by

Vijayalakshmi Kari

born in

Inkollu, India

Göttingen, 2013 


\section{Thesis Supervisor}

Prof. Dr. Steven A. Johnsen

\section{Doctorial Committee}

Prof. Dr. Steven A. Johnsen (Reviewer)

Department of Tumor Biology,

University Medical Center Hamburg Eppendorf, Hamburg

Prof. Dr. Holger Reichardt (Reviewer)

Dept. of Cellular and Molecular Immunology

Göttingen University Medical School, Göttingen

Prof. Dr. Ralph Kehlenbach

Institute for Biochemistry I

Göttingen University Medical School, Göttingen

Date of oral examination: $24^{\text {th }}$ June, 2013 


\section{Affidavit}

I hereby declare that the PhD thesis entitled "Role of Histone Metabolism and Chromatin Structure in DNA repair" has been written independently and with no other sources and aids than quoted.

Vijayalakshmi Kari

May, 2013

Hamburg 


\section{Table of contents}

List of Abbreviations .................................................................... I

List of Figures ............................................................................ V

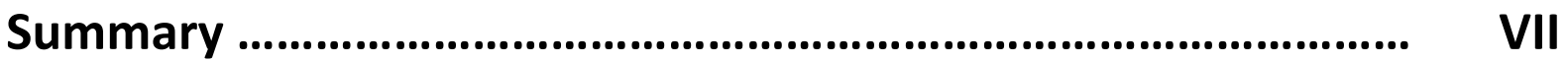

\section{General Introduction}

1.1 Chromatin structure and organization .......................................................... 1

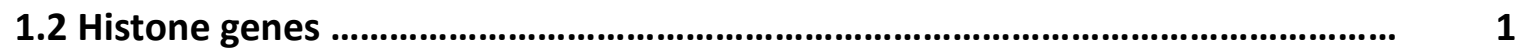

1.2.1 Replication-dependent histone genes ......................................................... 2

1.2.2 Transcription of replication-dependent histone genes .................................. 2

1.2.3 Replication-dependent histone mRNA processing ........................................ 3

1.2.4 Histone mRNA export, translation and degradation ..................................... 4

1.2.5 Polyadenylated replication-dependent histone mRNAs ............................... 5

1.2.6 Post-translational histone modifications and histone code ......................... 5

1.3 DNA damage and repair process ....................................................................... 7

1.3.1 Types of DNA damage and repair process ................................................... 8

1.3.2 DNA double stand break repair pathways ..................................................... 8

1.3.3 Non-homologous DNA end-joining (NHEJ) ................................................... 9

1.3.4 Homologous recombination (HR) pathway .................................................... 9

1.3.5 Alternative DSB repair pathways ............................................................... 10

1.4 DNA damage response (DDR) ........................................................................ 11

1.4.1 DNA damage sensors and mediators ........................................................... 12

1.4.2 DNA damage transducers .................................................................... 12

1.5 Histone modifications in DNA damage response (DDR) and repair .................. 13

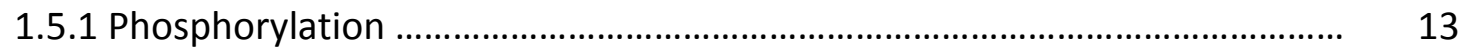

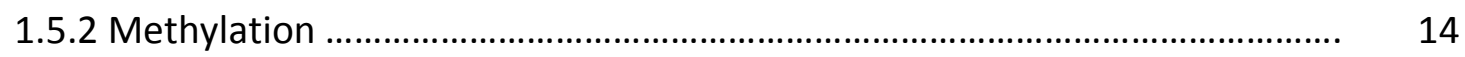

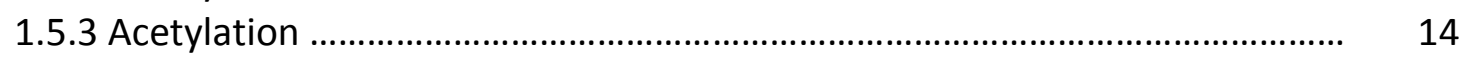

1.5.4 Ubiquitination ......................................................................................... 14

1.5.5 RNF20/40 and H2B mono-ubiquitination (H2Bub1) ................................... 15

1.6 ATP dependent chromatin remodeling complexes ........................................... 16

1.6.1 Chromodomain helicase DNA-binding (CHD) proteins in DNA repair ......... 17

1.7 Histone chaperones in DNA double stand beak repair ........................................ 18 


\section{Publications}

\subsection{Publication I}

The H2B ubiquitin ligase RNF40 cooperates with SUPT16H to induce dynamic changes in chromatin structure during DNA double-strand break repair

2.1.1 Supplementary Information

\subsection{Publication II}

A subset of histone $\mathrm{H} 2 \mathrm{~B}$ genes produces polyadenylated mRNAs under a variety of cellular conditions

2.2.1 Supplementary Information

\subsection{Manuscript in Preparation}

CHD1 is required for the homologous recombination repair pathway.

3. General Discussion

4. Reference List

5. Acknowledgements

6. Curriculum Vitae 


\section{List of Abbreviations}

${ }^{\circ} \mathrm{C}$

53BP1

36B4

7-AAD

ac

ASF

ATM

ATP

ATR

BER

BGP

BGS

bp

BRCA1

BrdU

Bre1p

BSA

CAF

CDK

CDNA

CHD

ChIP

$\mathrm{CHK}$

$\mathrm{CO}_{2}$

Con

CPSF

CPTF

CTD

CtIP

DMEM

DMSO

DNA

DNA-PKCS

Dot1L

DRS

DSBS

DTT

DUB

E1 enzyme
Degree Celsius / centigrade

p53 binding protein 1

Mouse homolog of human RPLPO gene

7-amino-actinomycin D

Acetylation

Anti-silencing function

Ataxia telangiectasia mutated

Adenosine triphosphate

Ataxia telangiectasia and Rad3 related

Base excision repair

B-Glycerolphosphate

Bovine growth serum

Base pair

Breast cancer 1

5-bromodeoxyuridine

Yeast brefeldin A sensitive protein 1

Bovine serum albumin

Chromatin assembly factor

Cyclin-dependent kinase

Complementary DNA

chromodomain helicase

Chromatin immunoprecipitation

checkpoint kinase

Carbon dioxide

Control

Cleavage and polyadenylation specificity factor

Cleavage stimulating factor

Carboxyterminal Domain

CTBP-interacting protein

Dulbecco/Vogt modified Eagle's minimal essential medium

Dimethyl sulfoxide

Deoxyribonucleic acid

DNA dependent protein kinase catalytic subunit

DOT1-like

Direct RNA sequencing

Double strand break

Dithiothreitol

Deubiquitinating enzyme

Ubiquitin-activating enzyme 


\begin{tabular}{|c|c|}
\hline E2 enzyme & Ubiquitin-conjugating enzyme \\
\hline E3 enzyme & Ubiquitin-ligase \\
\hline EDTA & Ethylenediaminetetraacetic acid \\
\hline e.g. & Exempli gratia = for example \\
\hline EIF & Eukaryotic translation initiation factor \\
\hline et al. & Etalii (and others) \\
\hline EXO1 & Exonuclease 1 \\
\hline $\mathrm{F}$ & Forward \\
\hline FACS & Fluorescence-Activated Cell Sorting \\
\hline FACT & Facilitates chromatin transcription \\
\hline FBS & Fetal bovine serum \\
\hline FCS & Fetal calf serum \\
\hline Fig & Figure \\
\hline g & Relative centrifugal force \\
\hline GAPDH & Glycerinaldehyd-3-phosphat-Dehydrogenase \\
\hline gDNA & Genomic DNA \\
\hline Gy & Gray \\
\hline h & Hour \\
\hline $\mathrm{H} 2 \mathrm{~A}$ & Histone 2A \\
\hline H2Aub1 & Monoubiquitinated histone $2 \mathrm{~A}$ \\
\hline $\mathrm{H} 2 \mathrm{~B}$ & Histone 2B \\
\hline H2Bub1 & Monoubiquitinated histone 2B \\
\hline H3 & Histone 3 \\
\hline H3K79me3 & Histone 3 trimethylated at position lysine 79 \\
\hline H3K56ac & Histone 3 acetylated at position lysine 56 \\
\hline $\mathrm{H} 4$ & Histone 4 \\
\hline HDE & Histone downstream element \\
\hline $\mathrm{HR}$ & Homologous recombination \\
\hline $\lg G$ & Immunoglobulin G \\
\hline ISWI & Imitation SWI2 \\
\hline K & Lysine residue \\
\hline $\mathrm{KCl}$ & Potassium chloride \\
\hline kDa & Kilo Dalton \\
\hline $\mathrm{L}$ & Leucine residue \\
\hline LSM & Like-sm protein \\
\hline $\mathrm{m}$ & $\operatorname{Milli}\left(10^{-3}\right)$ \\
\hline M & Molar, mol/L \\
\hline MDC1 & Mediator of DNA-damage checkpoint 1 \\
\hline MDM2 & Mouse double minute 2 \\
\hline me & Methylation \\
\hline $\mathrm{MgCl} 2$ & Magnesium chloride \\
\hline
\end{tabular}




\begin{tabular}{|c|c|}
\hline mRNAs & Messenger RNAs \\
\hline MRE11 & Meiotic recombination 11 \\
\hline MTA1 & Metastasis associated 1 \\
\hline $\mathrm{NaCl}$ & Sodium chloride \\
\hline NBS1 & Nijmegen breakage syndrome \\
\hline NCS & Neocarzinostatin \\
\hline NER & Nucleotide excision repair \\
\hline ng & Nanogram \\
\hline NHEJ & Non-homologous recombination \\
\hline NPAT & Nuclear protein ataxia-telangiectasia locus \\
\hline Nut & Nutlin-3a \\
\hline OCT1 & Octamer-binding protein 1 \\
\hline $\mathrm{p}$ & Phospho \\
\hline P53 & Tumor protein 53 \\
\hline PARP & Poly ADP ribose polymerase \\
\hline PCNA & Proliferating Cell Nuclear Antigen \\
\hline Pol & Polymerase \\
\hline PolyA & Polyadenylated \\
\hline PTMs & Post-translational modifications \\
\hline qRT-PCR & Quantitative real time PCR \\
\hline $\mathrm{R}$ & Reverse \\
\hline RAD & RecA homolog \\
\hline RNA & Ribonucleic acid \\
\hline RNF & Ring finger protein \\
\hline RPA & Replication protein A \\
\hline RPLPO & Ribosomal protein, large, P0 \\
\hline RPM & Revolutions per minute \\
\hline rRNAs & Ribosomal RNAs \\
\hline s.d. & Standard deviation \\
\hline SET & Superantigen-like protein \\
\hline siRNA & Small interfering RNA \\
\hline SLBP & Stem-loop binding protein \\
\hline SLIP1 & SLBP interacting protein 1 \\
\hline snoRNP & Small nucleolar ribonucleoprotein \\
\hline snRNA & Small nuclear RNA \\
\hline snRNP & Small nuclear ribonucleoprotein \\
\hline SSB & Single strand break \\
\hline SSRP & Structure specific recognition protein \\
\hline Suppl & Supplementary \\
\hline SuPT16 & Suppressor of Ty 16 \\
\hline SWI/SNF & SWItch/Sucrose Non-Fermentable \\
\hline
\end{tabular}




$\begin{array}{ll}\text { Tip60 } & \text { Tat-interactive protein-60 } \\ \text { TopBP1 } & \text { Topoisomerase binding protein } 1 \\ \text { tRNAs } & \text { Transfer RNAs } \\ \text { ub } & \text { Ubiquitination } \\ \text { UTR } & \text { Untranslated region } \\ \text { UV } & \text { Ultra violet } \\ \text { WAC } & \text { WW domain containing adaptor with coiled-coil region } \\ \text { XLF } & \text { Xrcc4 like factor } \\ \text { XRCC } & \text { X-ray cross-complementation group } \\ \mu & \text { Micro }\left(10^{-6}\right) \\ \nu & \text { Gamma }\end{array}$




\section{List of Figures}

\section{General Introduction}

Fig 1

Fig 2

Fig 3

Fig 4

Fig 5
The structure of a metazoan canonical histone mRNA

Post-translational modifications of core histones

Types of DNA repair mechanisms maintains genomic stability

Mammalian double-strand break (DSB) repair pathways

Chromatin restoration after DNA break repair

\section{Publication I}

Fig I.1

Fig I.2

Fig I.3

Fig I.4

Fig I.5

Fig I.6

Fig I.7

Fig 1.8

Fig 1.9

Suppl. Fig I.S1

Suppl. Fig I.S2
Expression of the histone $\mathrm{H} 2 \mathrm{~B}$ gene complement in different cell lines

Nutlin-3a treatment down-regulates the expression of normal replication-dependent histone $\mathrm{H} 2 \mathrm{~B}$ genes and up-regulates the expression polyA+ transcripts

Polyadenylation of histone $\mathrm{H} 2 \mathrm{~B}$ genes assigned using polyadenylation and alternative polyadenylation (APA) map

Expression of normal and PolyA+ HIST1H2BD and HIST1H2AC transcripts in HCT116 cells

Spliced HIST1H2BD and HIST1H2AC transcripts are transported to the cytoplasm and found on polyribosomes

Radiation induced elevated expression of spliced histone transcripts

Differentiation of uncommitted mesenchymal stem cells results in elevated expression of spliced up to histone transcripts

Expression of spliced histone transcripts are increased during committed osteoblast differentiation

Comparison of stem-loop sequences in $\mathrm{H} 2 \mathrm{~B}$ genes

Quality of polyA ${ }^{+}$mRNA purified using PolyA Ttract $^{\circledR}$ mRNA

Isolation System III

Purity of cytoplasmic and nuclear RNA

\section{Publication II}

Fig II.1 RNF40 knockdown leads to the prolonged $\mathrm{yH} 2 \mathrm{AX}$ accumulation and loss of H3K56ac increase after NCS treatment

Fig II.2

RNF40 is critical for cell cycle checkpoint activation

Fig II.3

RNF40 knockdown decreases the binding of SUPT16H and SSRP1

to chromatin

Fig II.4

RNF40 and SUPT16H are essential for RAD51 and RPA1 recruitment

to chromatin and DNA repair

Suppl. Fig II.S1 Loss of CHK2 activation can be partially rescued by overexpression of Flag-RNF40 in U2OS cells

Suppl. Fig II.S2

RNF40 and SUPT16H knockdown do not affect the expression of

one another or of the DNA repair proteins RPA1 and RAD51 


\section{Manuscript III}

Fig III.1 CHD1 is recruited to I-Scel-induced DSB site and is co-localized

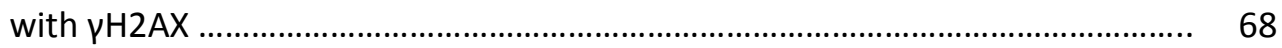

Fig III.2 CHD1 is localized to NCS induced DNA double strand break site ...................... 69

Fig III.3 CHD1 is recruited to the chromatin upon DNA double strand break induction

Fig III.4 CHD1 mediates cell cycle checkpoint activation in response to DNA double strand breaks

Fig III.5

CHD1 is required for homologous-recombination (HR) repair but not non- homologous end joining (NHEJ) pathway and cell survival after Mitomycin C treatment

Fig III.6 Depletion of CHD1 affects the end resection and the binding of

Fig III.7 RPA1 and RAD51 to chromatin in response to DNA DSB induction CHD1 affects end resection process probably through opening up the chromatin at DNA damage site 


\section{Summary}

During the cell cycle progression, synthesis of new histones is important to pack newly synthesized DNA and to maintain proper chromatin structure. Unlike normal mRNAs, mRNAs from replication-dependent histone genes that are expressed in the $\mathrm{S}$ phase of the cell cycle are not polyadenylated at the 3'end. They contain a conserved stem-loop sequence which forms a stem-loop structure which is required for the proper processing of the $3^{\prime}$ end, translation and degradation of histone mRNAs. However, a number of studies show that replication-dependent histone genes can produce mRNAs which have a polyA tail at the $3^{\prime}$ end under certain conditions the physiological function of polyadenylated histone mRNAs is not clear. In the present study, we analyzed the expression of polyadenylated histone mRNAs from the replication-dependent histone $\mathrm{H} 2 \mathrm{~B}$ genes. Furthermore, the expression of polyadenylated mRNAs from HIST1H2BD and HIST1H2AC genes are upregulated during differentiation and up on induction of DNA damage. We showed that polyadenylated HIST1H2BD and HIST1H2AC mRNAs are transported to the cytoplasm and can form polysomes suggesting that theses transcripts can be translated into proteins.

In addition to new synthesis of histone proteins, post-translational histone modifications, ATP-dependent chromatin remodelers and histone chaperones play important roles in maintaining genome structure and controlling DNA associated processes such as replication, transcription and DNA repair. Here we showed that one of the posttranslational histone modifications, the $\mathrm{H} 2 \mathrm{~B}$ monoubiquitination (H2Bub1) which was shown to be associated with actively transcribed genes, is important for DNA double strand break (DSB) repair. H2Bub1 is carried out by an E3 ubiquitin ligase complex RNF20/40 and knockdown of RNF40 leads to the loss of checkpoint activation. In addition, RNF40 also regulates the recruitment of the histone chaperone complex FACT to chromatin and is required for the chromatin dynamics at the DSB site. Further, we showed that CHD1 an ATPdependent DNA helicase is recruited to the site of DSB and regulates the binding of CtIP to chromatin. Depletion of CHD1 causes a decrease in homologous recombination-mediated repair efficiency and an increase in the cellular sensitivity to Mitomycin C treatment.

In summary, the data imply that E3 ubiquitin ligase RNF40 and chromatin remodeler CHD1 mediate DNA DSB repair through chromatin remodeling at the site of DNA damage. 


\section{General Introduction}

\subsection{Chromatin structure and organization}

Chromatin is composed of negatively charged DNA, positively charged histones and other proteins which regulate the functions of DNA. The basic unit of chromatin is a nucleosome which consists of 147 base pairs of DNA wrapped around a histone octamer including two copies of each $\mathrm{H} 3-\mathrm{H} 4$ heterodimer and two copies of core $\mathrm{H} 2 \mathrm{~A}-\mathrm{H} 2 \mathrm{~B}$ dimer (Kornberg, 1974). Nucleosomes are connected by linker DNA which is around 10-80 bp in length based on species and tissue and with linker histone $\mathrm{H} 1$. Nucleosomes associated with histone $\mathrm{H} 1$ tend to form higher order chromatin structure referred to as $30 \mathrm{nM}$ fibers (Allan et al., 1981; Thomas, 1999). However, the existence of the $30 \mathrm{nM}$ chromatin fiber structure in vivo is still debated. Histones are highly evolutionary conserved small basic proteins. The amino terminal (N-terminal) portion of histones is unfolded and protrudes away from the nucleosome where the central carboxyl terminal domains form the nucleosome scaffold (Hacques et al., 1990; Kornberg and Lorch, 1999; Luger et al., 1997). Chromatin is highly heterogeneous and broadly divided into two categories referred to as heterochromatin and euchromatin, based on the level of condensation and the accessibility of DNA (Mello, 1983). Heterochromatin is highly condensed and considered to be less active and contains fewer genes. In contrast to heterochromatin, euchromatin is less condensed and contains a higher density of genes either transcribed or repressed. However recent findings suggest that chromatin can also be categorized into different types based on other factors including structure, function and epigenetic makeup (Bickmore and van Steensel, 2013; Grewal and Elgin, 2007; Talbert and Henikoff, 2010).

\subsection{Histone genes}

In most of the eukaryotes two classes of histone genes are found 1) replicationdependent histone genes whose expression is regulated according to the cell cycle and 2) less abundant replication-independent histone genes that encode the minor histone variants and whose expression occurs at a basal level throughout the cell cycle ( $\mathrm{Wu}$ and Bonner, 1981a). 


\subsubsection{Replication-dependent histone genes}

In metazoans, the five canonical histone genes are organized in clusters that contain multiple copies of each in the genome (Marzluff et al., 2008). In mammals, replicationdependent histone genes are present in three clusters. The largest cluster called HIST1 located on chromosome 6 (6p21-6p22) in humans and chromosome 13 in mice, HIST2 located on chromosome 1 (1q21) and HIST3 located on chromosome 1 (1q42) in humans (Marzluff et al., 2002). Histone gene loci were found to be associated with Cajal bodies (CBs), subnuclear organelles that contain factors involved in $3^{\prime}$ processing of histone mRNAs (Frey and Matera, 1995; Wu and Gall, 1993). However, recent studies have revealed that histone gene clusters, as well as regulators of the histone expression and mRNA $3^{\prime}$ end processing are concentrated at subnuclear compartments called histone locus bodies (HLBs) (Ghule et al., 2008; Liu et al., 2006; Matera, 2006). HLBs are associated with replicationdependent histone gene clusters and their colocalization with CBs depends on the cell cycle phase (Bongiorno-Borbone et al., 2010).

\subsubsection{Transcription of replication-dependent histone genes}

Histones are essential for correct packing of DNA into chromatin. Thus, histones are produced rapidly in a high amount during S-phase in order to completely pack newly synthesized DNA into organized chromatin structure. Replication-dependent histone mRNA levels increase up to 10 -fold at the beginning of $S$ phase and come back to normal levels at the end of $S$ phase. The rapid increase in the histone mRNA levels at the beginning of $S$ phase is due to an increased transcription rate of histone genes while subsequent decrease is due to mRNA degradation at the end of S phase (DeLisle et al., 1983; Marzluff et al., 2008; Osley, 1991). Entry into $S$ phase is the trigger for increased transcription rate of histone genes. Several proteins are involved in regulation of replication-dependent histone transcription. One such protein is NPAT (nuclear protein ataxia-telangiectasia locus), which was shown to be essential for S phase entry and stimulation of histone gene transcription (Ye et al., 2003; Zhao et al., 2000). Cyclin E-CDK2 (cyclin-dependent kinase 2) phosphorylates NPAT at the beginning of S phase in the CBs and this results in increased transcription of canonical histone genes (Ma et al., 2000; Wei et al., 2003). The expression of the NPAT gene is regulated by E2F transcription factor family members where E2F1 and 
E2F4 play opposing roles to activate and repress NPAT transcription, respectively (Gao et al., 2003; Zhao et al., 1998). Another protein which is involved in histone mRNA transcription is OCT1 (octamer-binding protein 1 ) that binds directly to histone $\mathrm{H} 2 \mathrm{~B}$ promoters and activates transcription (Zheng et al., 2003).

\subsubsection{Replication-dependent histone mRNA processing}

Genetic information from DNA is transcribed into different forms of RNA molecules through RNA polymerases. Three types of RNA polymerases are known in eukaryotic cells. They are RNA polymerase (Pol) I which transcribes ribosomal RNAs (rRNAs), RNA Pol II which transcribes messenger RNAs (mRNAs) and as well as small regulatory RNAs and RNA Pol III which transcribes small RNAs such as transfer RNAs (tRNAs). Among these only mRNA molecules can be translated into proteins. A typical eukaryotic mRNA which is transcribed by RNA polymerase II contains a 5' cap, 5' untranslated region (UTR), coding region, 3' UTR and polyadenosine (polyA) tail. However, canonical replication-dependent histone mRNAs which are transcribed by RNA pol II are exceptional since they lack introns and are not polyadenylated (Dominski and Marzluff, 2007). In contrast, constitutively expressed histone mRNAs which encode histone variants are not cell cycle regulated, are polyadenylated and some even contain introns (Brush et al., 1985; Wells and Kedes, 1985).

Instead of a polyA tail at the $3^{\prime}$ end, replication-dependent histone mRNAs contain a highly conserved 16 nucleotide long sequence which forms stem-loop structure. Upstream to the stem-loop there is a cleavage site which is $4-5 \mathrm{nt}$ and followed by a purine-rich histone downstream element (HDE) (Birnstiel et al., 1985; Dominski and Marzluff, 2007; Marzluff, 1992) (Fig. 1). Processing of $3^{\prime}$ end requires the binding of stem-loop binding protein (SLBP) to the conserved stem-loop sequence and U7 snRNA (small nuclear RNA), component of snRNP (small nuclear ribonucleoprotein) with HDE (Dominski and Marzluff, 2007; Mowry and Steitz, 1987). SLBP and the U7 snRNP together recruit a cleavage factor complex that contains CPSF73 (cleavage and polyadenylation specificity factor 73), CPSF100 and Symplekin1 that also processes the $3^{\prime}$ end of polyadenylated mRNAs (Kolev and Steitz, 2005; Kolev et al., 2008). Structure of $3^{\prime}$ histone mRNA and its processing factors are illustrated in Figure 1. 
a
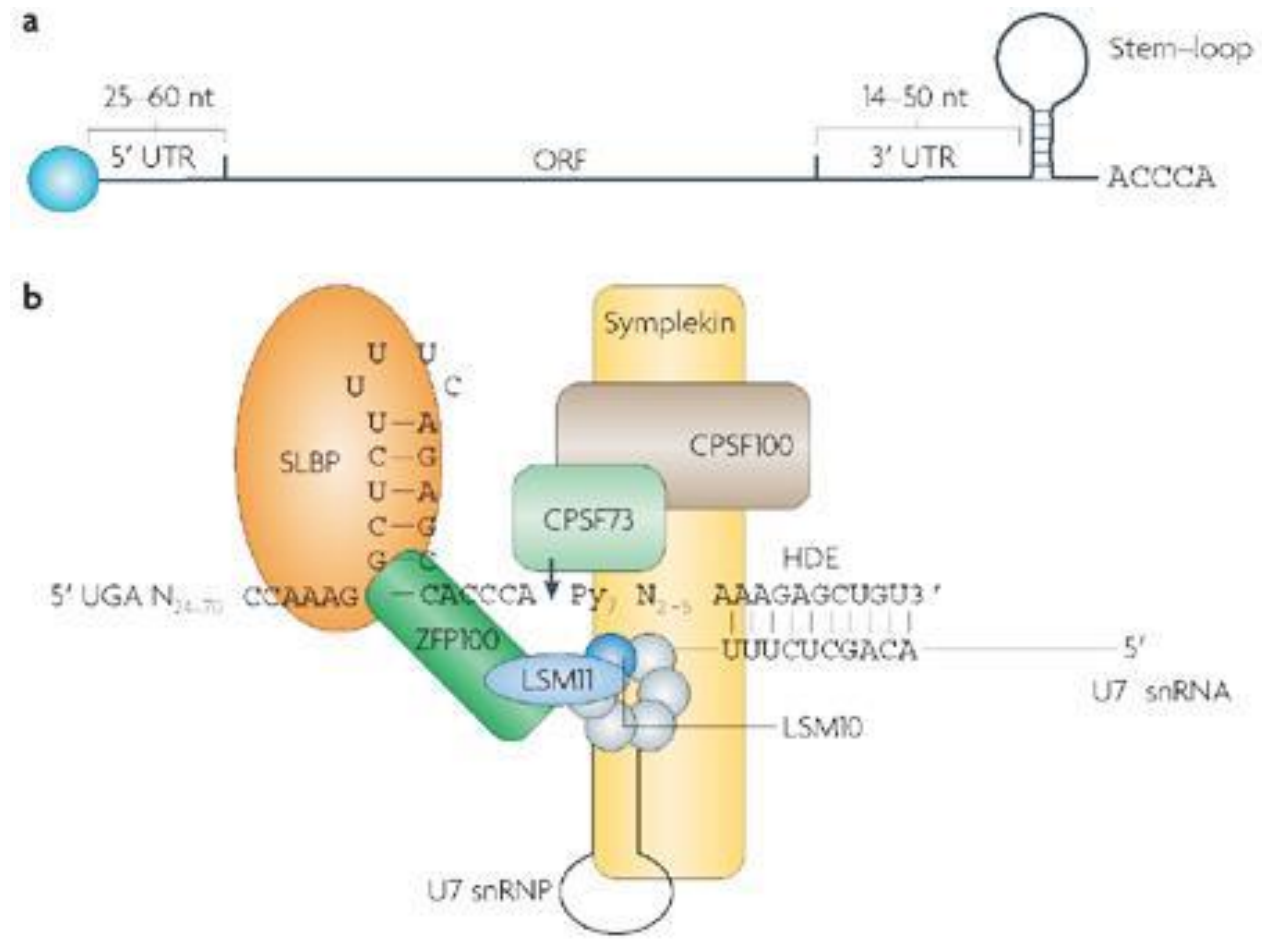

Fig 1. The structure of a metazoan canonical histone mRNA. A) The structure of typical histone mRNA that lack introns and have short 5' and 3' UTRs. B) Processing of mammalian canonical histone pre-mRNA. Histone pre-mRNAs contain a conserved stem-loop sequence that binds stem-loop binding protein (SLBP) followed by the histone downstream element (HDE), which base-pairs with U7 small nuclear RNA (snRNA). A cleavage complex containing CPSF73 (cleavage and polyadenylation specificity factor subunit 73), CPSF100 and Symplekin is recruited to cleave the pre-mRNA. The cleavage (arrow), which occurs at five nucleotides downstream of the stem-loop and upstream of the HDE (Marzluff et al., 2008).

\subsubsection{Histone mRNA export, translation and degradation}

Like other mRNAs, replication-dependent histone mRNAs are exported from the nucleus to cytoplasm for translation into proteins by antigen peptide transporter (Erkmann et al., 2005; Huang and Steitz, 2001). The SLBP protein, which binds to stem-loop structure, also helps in the transport of histone mRNA and in the initiation of translation. It was shown that SLBP interacting protein 1 (SLIP1) interacts with eukaryotic translation initiation factor $4-\gamma$ (EIF4G) to bring the $3^{\prime}$ end proximal to the $5^{\prime}$ cap to initiate and allow efficient translation (Cakmakci et al., 2008). When cells approach the end of S phase, the level of replication-dependent histone transcripts starts decreasing which is achieved by a rapid reduction in mRNA half-life in mammals. SLBP is also involved in the process of degradation by recruiting proteins responsible for a adding short oligo $(U)$ tail to the histone mRNA that is being translated (Mullen and Marzluff, 2008; Osley, 1991). Then the LSM1-7 heptamer is 
recruited and promotes the degradation of histone mRNAs similar to polyadenylated mRNAs (Tharun et al., 2000).

\subsubsection{Polyadenylated replication-dependent histone mRNAs}

The existence of polyadenylated histone mRNAs from replication-dependent histone genes has been reported. Using a computational approach it was shown that many histone mRNAs contain both polyadenylation signal as well as stem-loop sequences (Dávila López and Samuelsson, 2008). Moreover, the loss of normal histone mRNA 3' end processing by depletion of SLBP or U7 SnRNP can result in the production of polyadenylated histone mRNAs (Sullivan et al., 2009). Similarly, depleting CDK9, CBP80 (Cap Binding Protein) or NPAT also results in the production of polyadenylated histone mRNAs (Narita et al., 2007; Pirngruber et al., 2009a). Earlier studies have shown that during differentiation and cell cycle arrest levels of polyadenylated histone mRNAs increased (Abba et al., 2005; Kirsh et al., 1989; Zhao et al., 2004). Moreover, microarray based studies indicate that the expression of several histone genes are upregulated during tumor progression since these studies were based on an initial polyT reverse transcription they probably indicate changes in histone mRNA polyadenylation rather than transcriptional changes. Tumor suppressor protein p53-mediated G1 cell cycle arrest also increases the fraction of polyadenylated histone transcripts in the cells (Pirngruber and Johnsen, 2010). However, whether polyadenylated histone mRNAs produced from normally replication-dependent histone genes are transported from the nucleus to the cytoplasm and engaged by ribosome remains unclear.

\subsubsection{Post-translational histone modifications and histone code}

In the cell, chromatin is dynamic and undergoes structural changes to facilitate various DNA associated processes. Chromatin associated processes are partially regulated by post-translational histone modifications (PTMs) which occur largely at the N-terminal tail of histones. These include acetylation, phosphorylation, ubiquitination, methylation, sumoylation and ADP ribosylation (Cosgrove and Wolberger, 2005; Fischle et al., 2003; Kouzarides, 2007) (Figure 2). 


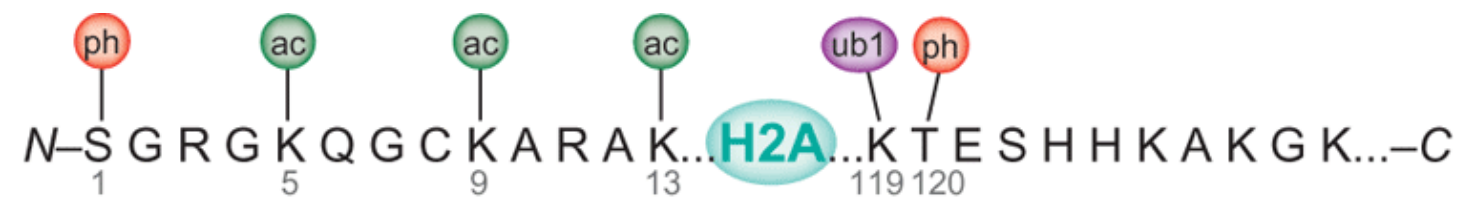

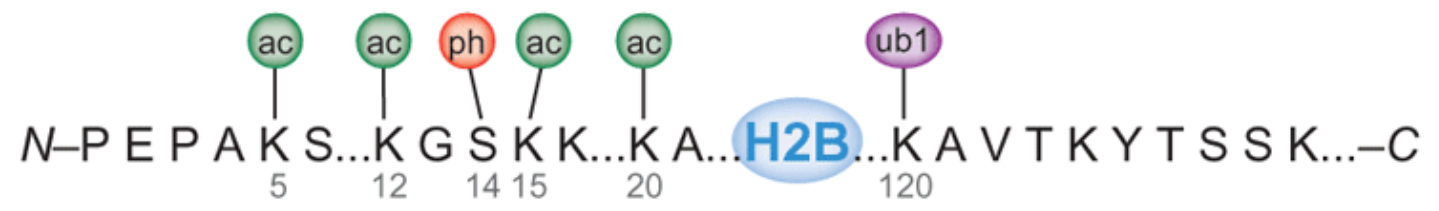

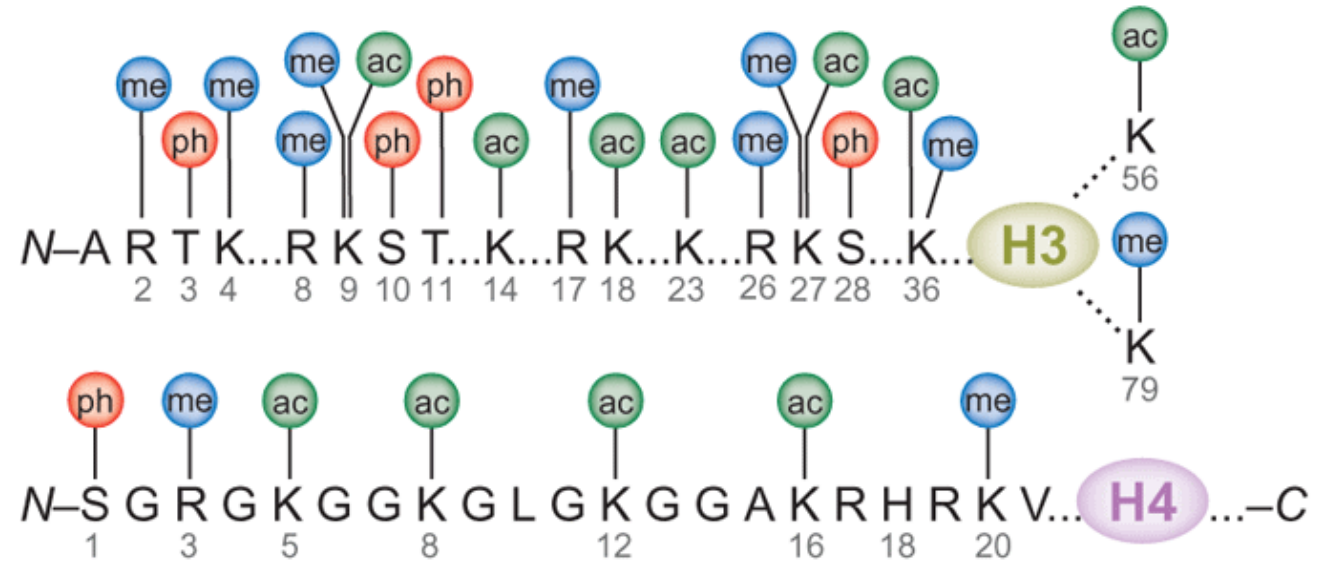

Fig 2. Post-translational modifications of core histones. Histone tails can be posttranslationally modified defining the state of chromatin (Bhaumik et al., 2007). ac acetylation, me - methylation, ph - phosphorylation, ub-ubiquitination.

Histone PTMs influence the chromatin state in mainly two ways, either by changing the electrostatic charges between DNA and histone (e.g. acetylation or phosphorylation) or by creating a platform for chromatin binding proteins (Berger, 2007; Strahl and Allis, 2000). The type of histone modification, number, combination, position on the histone dictates the outcome of DNA associated processes, referred to as the "histone code" (Berger, 2002; Jenuwein and Allis, 2001). Histone modifications can also influence each other by "histone crosstalk". Importantly, some marks are generally associated with active chromatin whereas some with a repressed state. 


\subsection{DNA damage and repair process}

Genetic information is stored and transferred to generations in the form of DNA, maintaining its integrity and stability which is essential for life. DNA constantly undergoes alterations by cellular pathways and is exposed to number of agents that cause damage to the DNA. DNA damage could occur by both endogenous processes and exogenous agents, which may cause deletions, mutations and chromosomal translocations in the genome. DNA damage may trigger a cascade of events including the sensing the DNA damage, activation of cell cycle checkpoint pathways, DNA repair and termination of DNA damage response signaling. If a cell is not able to repair the breaks an alternative apoptosis pathway becomes activated. Any defects in these processes can lead to genomic instability, aging and diseases such as cancer.

\subsubsection{Types of DNA damage and repair process}

DNA damage can be caused by endogenous processes that deal with the DNA such as replication, transcription and by exogenous agents including chemical compounds, UV rays and $X$ rays. For example, intrinsic processes like replication may lead to mismatches and strand breaks; chemical agents like Cisplatin cause inter and intra strand crosslinks, UV exposure creates pyrimidine dimers and base modifications; ionizing radiation exposure leads to abasic sites, single and double strand breaks. Both prokaryotic and eukaryotic cells have evolved different types of DNA repair mechanisms to repair a variety of DNA adducts and damage (Ciccia and Elledge, 2010; Lord and Ashworth, 2012; Shiloh, 2003) and most of the processes are evolutionary conserved. Single strand breaks (SSB) are repaired by the base excision repair (BER) pathway; bulky adducts created by exposure to UV are repaired through nucleotide excision repair (NER); mismatches, insertions or deletions are removed by the mismatch repair pathway; most lethal DNA double strand breaks are repaired by a homology mediated repair pathway called homologous recombination (HR) or by an errorprone non-homologous end joining (NHEJ) pathway (Jiricny, 2006; Lindahl and Barnes, 2000; Moldovan and D'Andrea, 2009; West, 2003). Well studied types of DNA damage and their repair mechanism and proteins involved in that pathways are shown in Figure 3. 


\subsubsection{DNA double stand break repair pathways}

DNA double strand breaks (DSBs) caused by endogenous processes or exogenous agents can promote genomic rearrangements and apoptosis. To repair DSBs multiple pathways have evolved. Two major pathways that have been studied well are nonhomologous DNA end-joining (NHEJ) and homologous recombination (HR) and other pathways like, alternative-NHEJ (alt-NHEJ) and single-strand annealing (SSA) (Ciccia and Elledge, 2010).

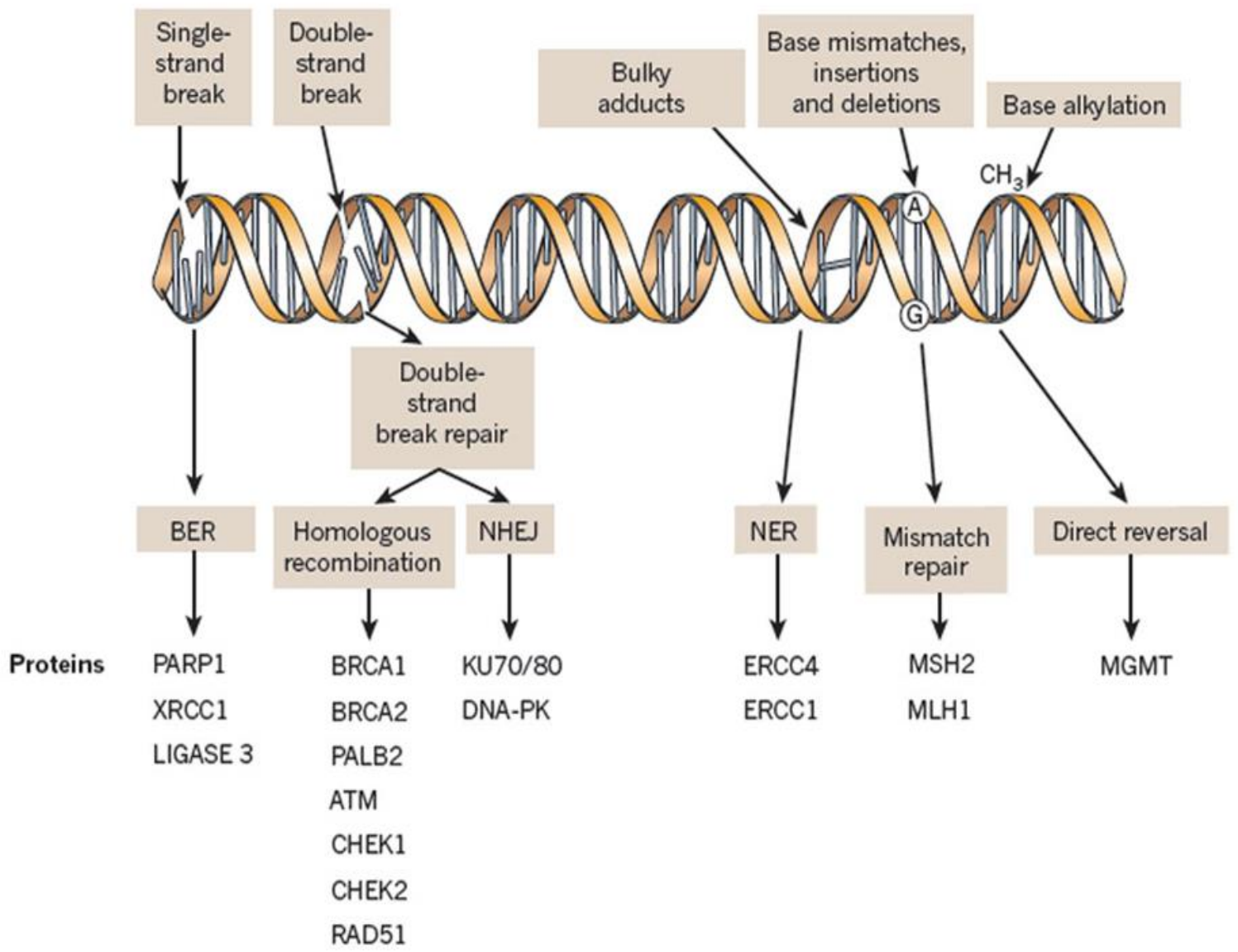

Fig 3. Types of DNA repair mechanisms maintain genomic stability. DNA is continually exposed to a number of agents that cause different types of damages, from single-strand breaks (SSBs) to base alkylation events. The choice of repair mechanism is largely based on the type of damage, but factors such as the stage of the cell cycle also have a role. BER, base excision repair; NER, nucleotide excision repair; NHEJ, non-homologous end joining. (Modified from (Lord and Ashworth, 2012)). 


\subsubsection{Non-homologous DNA end-joining (NHEJ)}

NHEJ repairs double strand breaks with little or no homology mediated pathway. In NHEJ, the two broken ends of DNA are held together with a DNA binding heterodimer protein complex called Ku70/80 and this recruits the catalytic subunit of DNA dependent protein kinase (DNA-PKcs) to the damage site (Gottlieb and Jackson, 1993). Upon binding DNA-PKCs is autophosphorylated which in turn promotes phosphorylation of its targets including NHEJ factors (Chan et al., 1999; Goodarzi et al., 2006; Leber et al., 1998). DNA-PKcs has an important role in stabilizing the ends of the DNA and preventing excessive end resection (Mahaney et al., 2009; Meek et al., 2008). The ends can be trimmed by a nuclease called Artemis and gaps are filled by DNA polymerases such as Pol $\mu$ or Pol $\lambda$ and allow the joining of the ends by the ligase complex including DNA ligase IV, X-ray crosscomplementation group 4 (XRCC4) and Xrcc4 like factor (XLF)/Cernunnos ligase (Critchlow and Jackson, 1998). The NHEJ pathway is active throughout the cell cycle but is favored in G1 cells and seals the ends very quickly. However, this pathway is error-prone and frequently results in insertions, deletions and substitutions. This pathway is also involved in $V(D) J$ recombination, the process required for the generation of antibody diversity in immune cells (Bassing et al., 2002). NHEJ is also responsible for chromosomal translocations if two different chromosome parts are joined together (Brandsma and Gent, 2012; Lieber, 2010; Ma et al., 2005).

\subsubsection{Homologous recombination (HR) pathway}

The HR pathway requires homologous sequences or a homology partner to repair DNA double strand breaks. This pathway is active in both $\mathrm{S}$ and the $\mathrm{G} 2$ phases of the cell cycle (Mao et al., 2008; Sung and Klein, 2006). Briefly, HR starts with resection of DNA ends by MRE11/RAD50/NBS1 (MRN complex) together with CtIP and exonucleases by generating 3' single-strand DNA (ssDNA) (Heyer et al., 2010; Limbo et al., 2007; Stracker and Petrini, 2011). Then the ssDNA is coated with ssDNA binding proteins replication protein A (RPA1) and RAD51 (Sugiyama et al., 1997) which recognizes homology and promotes strand invasion in order to copy information from the donor chromosome. The central part of HR is the formation of Holliday junction (Collins and Newlon, 1994; Sung and Klein, 2006). 
After recognizing DSBs the MRN complex promotes activation of ATM (ataxia telangiectasia mutated) kinase. The MRN complex includes structural maintenance of chromosome (SMC) family member RAD50 which further interacts with MRE11 and associates with DSBs to stabilize the ends. MRE11 also has both endonuclease and exonuclease activity important for the initial end resection process (Williams et al., 2007). The third member of the MRN complex is NBS1, which interacts with MRE11 as well as with other proteins involved in the DNA damage response (DDR) pathway such as ATM, CTBPinteracting protein (CtIP) and mediator of DNA damage checkpoint 1 (MDC1) (van den Bosch et al., 2003). ATM was shown to regulate end resection process through CtIP, which interacts with BRCA1 and MRN complex in S and G2 phase of cell cycle (Huen et al., 2010). Further end resection process is carried out by EXO1 which is also phosphorylated by ATM (Bolderson et al., 2010). It is reported that DNA Polymerase $\delta$ is required for HR mediated DNA synthesis (Maloisel et al., 2008).

\subsubsection{Alternative DSB repair pathways}

Apart from the two major pathways to repair DSBs (HR and NHEJ), there are other pathways which have been reported such as the single strand annealing (SSA) pathway which uses directly repeated stretches of homology to repair DSBs and the alternative NHEJ pathway (A-NHEJ). The later was reported to be active when the classical NHEJ is inactive and is similar to the NHEJ pathway in that it is also an error-prone. Proteins involved in this pathway are DNA ligase III, XRCC1 and PARP1 (Audebert et al., 2004; Wang et al., 2006). While a number of pathways to repair DSBs have been reported, recent studies indicate that all the repair pathways are interconnected with each other. The pathway of choice depends on cell cycle phase and availability of regulatory proteins. 


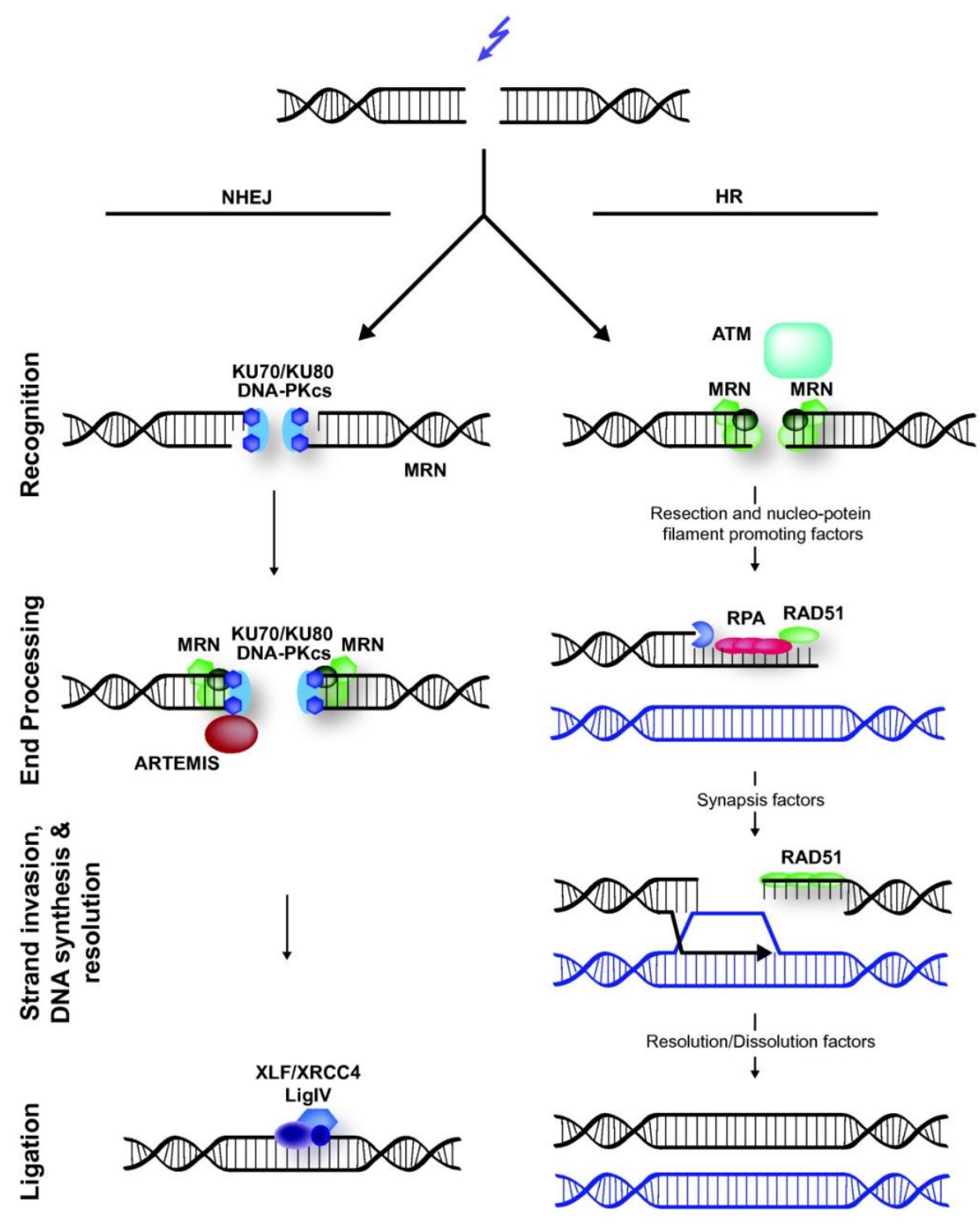

Fig 4. Mammalian double-strand break (DSB) repair pathways. DNA DSBs are predominantly repaired by either non-homologous end-joining (NHEJ) or homologous recombination (HR) (Lans et al., 2012).

\subsection{DNA damage response (DDR)}

Cells have evolved sophisticated mechanisms to recognize different types of DNA damage and activate cell cycle checkpoints to transduce signals to repair the DNA damage. The DNA damage response (DDR) pathway involves sensing the DNA damage by sensors and mediates a cascade of events to protect cells (Harper and Elledge, 2007; Jackson and Bartek, 2009) from damage. Following sections will introduce the molecules involved in the DNA damage response. 


\subsubsection{DNA damage sensors and mediators}

Once DNA is damaged in the cell, the first step is the recognition of DNA damage to initiate the checkpoint activation and repair. Studies from both yeast and mammals demonstrated that Rad9, Rad1, Hus1 (9-1-1) and Rad17 are the essential factors that activate checkpoint signaling (Longhese et al., 1998; Parrilla-Castellar et al., 2004). The 9-1-1 complex facilitates the activation of the ATM/ATR kinases (Kondo et al., 2001). DNA damage mediators are BRCA1 C-terminus repeat domain (BRCT) containing proteins that mediate protein-phosphoprotein interactions including p53 binding protein 1 (53BP1), MDC1, MRN complex, topoisomerase binding protein 1 (TopBP1) and breast cancer susceptibility gene 1 (BRCA1) (Goldberg et al., 2003; Schultz et al., 2000; Wang et al., 2002; Yamane et al., 2002). Recruitment of mediators is mostly dependent on phosphorylation of $\mathrm{H} 2 \mathrm{AX}(\mathrm{\gamma H} 2 \mathrm{AX})$ modification which occurs on both sides of DNA damage site by Phosphatidylinositol 3kinase-like protein kinase (PIKK) family members ATM and ATR (Rogakou et al., 1998a).

\subsubsection{DNA damage transducers}

The DNA damage response is mediated primarily by kinases that belong to phosphatidylinositol 3-kinase-like protein kinase (PIKKs) family which includes ataxia telangiectasia mutated (ATM), ataxia telangiectasia and Rad3-related protein (ATR) and DNA-dependent protein kinase catalytic subunit (DNA-PKcs). Recently it was also shown that proteins belonging to the poly ADP ribose polymerase family (PARP1 and PARP2) (Cimprich and Cortez, 2008; Savitsky et al., 1995; Schreiber et al., 2006; Walker et al., 1985) are also required for DNA damage response. The ATM/ATR-mediated DNA damage response pathway is very well studied. ATM is a large ( 350-kDa), evolutionarily conserved serine/threonine protein kinase and is activated in response to DNA double strand breaks (DSBs). The DNA damage sensor MRN complex consisting of MRE11, RAD50 and NBS1 has been implicated as one of the initial activators of ATM (Cerosaletti and Concannon, 2004; Lee et al., 2013; Uziel et al., 2003). Other proteins are also implicated in ATM activation including RNF8 (ring finger protein 8) an E3 ubiquitin ligase and BRCA1-associated ATM activator 1 (BAAT1) (Ouchi and Ouchi, 2010; Wu et al., 2011). ATM exists as a dimer which is inactive, once it is recruited to the damage site it becomes a monomer and is autophosphorylated at multiple sites (Ser367, Ser1893, Thr1885, Ser1981 and Ser2996). This 
is thought to be important for further activation and retention of ATM at the damage site (Bakkenist and Kastan, 2003; Kozlov et al., 2011; You et al., 2007). Once ATM is activated in response to DNA damage, it phosphorylates a number of proteins involved in cell cycle checkpoint control, DNA repair and apoptosis such as H2AX, CHK2, NBS1, BRCA1, p53, MDM2, SMC1 and others (Banin et al., 1998; Lim et al., 2000; Matsuoka et al., 2007; McKinnon, 2012). Downstream of these proteins are checkpoint kinases (CHK) CHK1 and CHK2 and their homologues. Mutations in the ATM gene cause an autosomal recessive neurodegenerative disease called A-T (Ataxia-telangiectasia). These patients exhibit hypersensitivity to radiation, immune dysfunction and early onset of cancer development (Gatti et al., 2001; Taylor and Byrd, 2005). ATR with its regulator ATRIP (ATR-interacting protein) senses single strand DNA (ssDNA) breaks generated by exposure to UV radiation or stalled replication fork. Though the main substrate of ATR is CHK1, most of the ATR substrates overlap with ATM including H2AX, BRCA1 and p53 (Cimprich and Cortez, 2008; Matsuoka et al., 2007; Tibbetts et al., 1999). DNA-PKcs is also autophosphorylated at different sites in response to DSBs and is mostly implicated in DNA repair through the NHEJ pathway rather than DNA damage response pathway (Meek et al., 2008).

\subsection{Histone modifications in DNA damage response (DDR) and repair}

Post-translational histone modifications (PTMs) not only regulate gene transcription, but are also involved in DNA damage recognition and repair pathway (Altaf et al., 2007; Rossetto et al., 2010).

\subsubsection{Phosphorylation}

An important histone modification involved in DNA repair is phosphorylation of $\mathrm{H} 2 \mathrm{AX}(\gamma \mathrm{H} 2 \mathrm{AX})$ at Ser139 by ATM, ATR and DNA-PKcs in response to DNA damage. This modification can spread around the damage site more than $50 \mathrm{~kb}$ (Downs et al., 2004; Rogakou et al., 1998a; Stiff et al., 2004). Cells lacking H2AX show sensitivity to ionizing radiation and genomic instability (Bassing et al., 2002; Celeste et al., 2002). $\mathrm{yH} 2 \mathrm{AX}$ is not only a DNA damage sensor but is also required for the binding of a number of proteins to the damage site including the MRN complex, MDC1, 53BP1 and others by directly 
interacting with them. Histones are also phosphorylated at other sites in response to DNA damage such as phosphorylation of $\mathrm{H} 2 \mathrm{~A}$ at Ser119, H2B at Ser14 and H4 at Ser1 (Cheung et al., 2005; Fernandez-Capetillo et al., 2004; Harvey et al., 2005). However, the functions of these in this process remain largely unknown.

\subsubsection{Methylation}

While histone methylation is thought to be important for transcriptional regulation recent studies have implicated numerous histone methylations in the DNA damage repair processes. Studies on the histone methyltransferase SET8, which mediates H4K20 methylation, revealed the role of this modification in maintaining genomic stability. Loss of SET8 leads to accumulation of DSBs and checkpoint activation (Jørgensen et al., 2007; Oda et al., 2009). The DNA damage mediator protein, 53BP1 can also interact with H3K79me which is mediated by DOT1-like (Dot1L) and H4K20me at site of the DNA damage through its TUDOR domains (Sanders et al., 2004).

\subsubsection{Acetylation}

Acetylation of histones seems to play a major role both in recruiting proteins to damage site and restoration of chromatin structure after the repair. A multi subunit complex containing the histone acetyltransferase TIP60 was shown to acetylate H2AX at Lys5 in response to DNA damage and ATM activation (Ikura et al., 2000). Acetylation and deacetylation of H4K16 plays a key role in the regulation of the DDR and is directly linked to the unfolding of higher-order chromatin structures (Shogren-Knaak et al., 2006). However, some histone acetylation marks were shown to decrease in response to DNA damage such as H3K9 and H4K16 acetylation. There are some controversial observations in case of H3K56 acetylation where some studies show that it is increased upon DNA damage others showing the opposite results (Tjeertes et al., 2009; Vempati et al., 2010).

\subsubsection{Ubiquitination}

Ubiquitination is a covalent modification involving the addition of ubiquitin to the target proteins. While polyubiquitination of proteins generally leads to protein degradation, monoubiquitination of a protein may change its function, localization or interacting partners (Bergink and Jentsch, 2009). Ubiquitination is carried out in a step wise process by three 
enzymes known as E1 ubiquitin-activating enzyme, E2 ubiquitin-conjugating enzyme and E3 ubiquitin ligase (Jackson and Durocher, 2013). Like other modifications ubiquitination is reversed by deubiquitinating (DUBs) enzymes. Ubiquitination of histones is not only important for gene regulation but also required for DDR pathway. For example the E2 conjugating enzyme Rad6 and the E3 ligase Rad18 are involved in post-replication repair (PRR) and translesion synthesis (TLS) by mono or poly ubiquitinating PCNA (Bergink and Jentsch, 2009; Ulrich, 2011). Monoubiquitination of histones and polyubiquitination of DDB2 and XPC is important for NER mediated repair pathway (Scrima et al., 2011). Most importantly, ubiquitination of histones during DNA double strand break response and repair play a major role in maintaining genomic stability. RNF8 and RNF168 mediated ubiquitination of histone $\mathrm{H} 2 \mathrm{AX}$ and $\mathrm{H} 2 \mathrm{~A}$ at $\mathrm{K} 119$ is required for retention of DSB repair and signaling factors such as 53BP1, RAD18, BRCA1, the RAP80 complex (also known as BRCA1A), HERC2, BMI1, RIF1, RNF169, NPM1, FAAP20, and NIPBL (Lukas et al., 2011). We and others have shown that $\mathrm{H} 2 \mathrm{~B}$ monoubiquitination at Lys 120 (H2Bub1) mediated by RNF20/40 E3 ligase regulates the chromatin remodeling at DNA damage site (Kari et al., 2011; Moyal et al., 2011; Nakamura et al., 2011). More details about H2B monoubiquitination will be discussed in further sections.

\subsubsection{RNF20/40 and H2B mono-ubiquitination (H2Bub1)}

H2B monoubiquitination is mediated by the RNF20/40 E3 ubiquitin ligase complex at Lys120 (K120) in mammals and by Bre1 at Lys123 in yeast. H2Bub1 is preferentially found in the transcribed region of highly expressed genes and at the promoter regions of some genes (Minsky et al., 2008) and the occupancy is overlapped with Ser2 phosphorylated form of RNAPII at p21 gene locus (Pirngruber et al., 2009a). In both yeast and human, it is linked with transcriptional elongation (Fleming et al., 2008; Minsky et al., 2008). During transcription RNF20/40 is recruited through interaction with WAC protein which directly interacts with Ser2 phospho RNA pol II (Zhang and Yu, 2011). Moreover, H2Bub1 is required to remove the repressive mark H3K27me3 at differentiation regulated genes (Karpiuk et al., 2012). Yeast Bre1 was shown to be recruited to chromatin via interaction with Paf1 complex (Henry et al., 2003; Xiao et al., 2005; Zhang and Yu, 2011). Furthermore, Paf1 interaction with facilitates chromatin transcription (FACT) complex facilitates removal of $\mathrm{H} 2 \mathrm{~A}-\mathrm{H} 2 \mathrm{~B}$ dimer from core nucleosomes (Belotserkovskaya et al., 2003; Kireeva et al., 2002; Laribee et 
al., 2007). H2Bub1 has been shown to promote the activity of the histone methyltransferases Set1 and Dot1L to di- and trimethylate H3K4 and H3K79 thereby facilitating histone crosstalk in yeast and mammals (Kim et al., 2009; Sun and Allis, 2002). Furthermore, H2Bub1 was shown to be required for correct processing of stem-loop dependent histone gene transcription (Pirngruber et al., 2009a).

Apart from the role in transcription, recent studies show that $\mathrm{H} 2 \mathrm{Bub1}$ is required for the DSB repair pathway. This modification was shown to increase following DNA damage in an ATM dependent manner (Moyal et al., 2011; Nakamura et al., 2011). RNF20 and RNF40 are phosphorylated by ATM in response to DNA damage and loss of this complex leads to decreased cell cycle checkpoint activation and chromatin accessibility (Chernikova et al., 2010; Kari et al., 2011; Moyal et al., 2011; Nakamura et al., 2011). Moreover, depletion of these two proteins leads to decreased efficiency of NHEJ and HR pathway (Moyal et al., 2011; Nakamura et al., 2011).

\subsection{ATP dependent chromatin remodeling complexes}

Like most of the DNA-associated processes in eukaryotes to access the DNA, DSB repair pathway also must deal with chromatin structure. The impact of chromatin on DNA repair was initially described in the "access-repair-restore" model (Smerdon, 1991). Modulation of chromatin compaction can be regulated by post-translational histone modifications, ATP-dependent chromatin remodeling complexes and histone chaperones. ATP-dependent chromatin remodeling complexes namely SWI/SNF, ISWI, CHD and INO80 are multi-protein complexes which use ATP as a source of energy to induce changes in the chromatin. Chromatin remodelers allow proteins to access DNA either by removing nucleosomes from chromatin to increase DNA accessibility shift the position of nucleosomes or exchange histones (Price and D'Andrea, 2013). In yeast, all four families of proteins have been shown to be involved in remodeling chromatin around the DSB site (Chai et al., 2005; Downs et al., 2004; Tsukuda et al., 2005). In humans, a well-studied chromatin remodeling complex at DSB site is hNuA4 which belongs to the INO80 family, consists of at least 16 subunits including the Tip60 acetyltransferase, p400 motor ATPase, Ruvbl1 and Ruvbl2 helicase-like proteins etc., (Jha et al., 2008; Sun et al., 2005; Xu et al., 2010). Tip60 acetylates chromatin and DDR proteins including ATM and p53 (Bird et al., 2002; Ikura et al., 2000; Sun 
et al., 2005). In mammals, hNuA4 can also promote the rapid exchange of H2A for H2A.Z at DSBs (Xu et al., 2012). Furthermore, it was shown that inactivation of hNuA4 components can lead to defects in the recruitment of other DDR proteins including RNF8/RNF168, 53BP1 and Rad51 to chromatin (Courilleau et al., 2012; Murr et al., 2007).

Another ATP-dependent chromatin remodeling SWI/SNF complexe belongs to the family of swi2/snf2 and it is also known to regulate chromatin structure in DNA repair. In mammals, it is recruited to DNA damage site via interaction with $\mathrm{\gamma H} 2 \mathrm{AX}$. Down-regulation of its components BRG-1, results in inefficient DSB repair and increased damage sensitivity (Lee et al., 2010; Park et al., 2006).

\subsubsection{Chromodomain helicase DNA-binding (CHD) proteins in DNA repair}

Chromodomain helicase DNA-binding (CHD) proteins belong to ATP-dependent chromatin remodelers and contain a tandem domain at the $\mathrm{N}$ terminal region and SNF2 like ATPase domain (Delmas et al., 1993; Woodage et al., 1997). A number of CHD proteins were known from different studies including CHD1 to CHD9 (Marfella and Imbalzano, 2007). CHD1 was initially identified in mouse (mChd1) and is co-purified along with SSRP1 the subunit of the FACT complex (Kelley et al., 1999). A recent study in yeast identified chd1 as a part of the SAGA and SAGA-like (SLIK) complexes required for proper histone acetyltransferase (HAT) activity (Pray-Grant et al., 2005). CHD1-like protein (CHD1L) was shown to be involved in DNA repair (Ahel et al., 2009). CHD4 is a part of hNuRD complex identified as a target for ATM/ATR-mediated phosphorylation and interacts with ATR (Matsuoka et al., 2007; Schmidt and Schreiber, 1999). Further, CHD4 was shown to be recruited to laser-induced DNA damage site and loss of CHD4 increased sensitivity of cells to IR (Larsen et al., 2010).

In this study, we show that CHD1 is recruited to DSB sites and required for end resection process. Moreover, depletion of CHD1 leads to inefficient repair of DSB through the homologous recombination repair pathway and affects cell survival. 


\subsection{Histone chaperones in DNA double stand beak repair}

Histone chaperones are proteins that allow ordered formation of nucleosomes and shield nonspecific interactions between histones and nucleic acids. During the assembly of nucleosomes, H2A-H2B dimers deposited on DNA only after the deposition of two H3-H4 dimer. During chromatin disassembly $\mathrm{H} 2 \mathrm{~A}-\mathrm{H} 2 \mathrm{~B}$ dimers are removed from the nucleosomes prior to $\mathrm{H} 3-\mathrm{H} 4$ dimer eviction. The role of DNA histone chaperones is well studied during the replication. Recent studies showed that histone chaperones are also involved in both chromatin assembly and disassembly during the DNA damage response and repair pathway and are mostly important in restoration of chromatin after repair (Ransom et al., 2010; Rossetto et al., 2010).

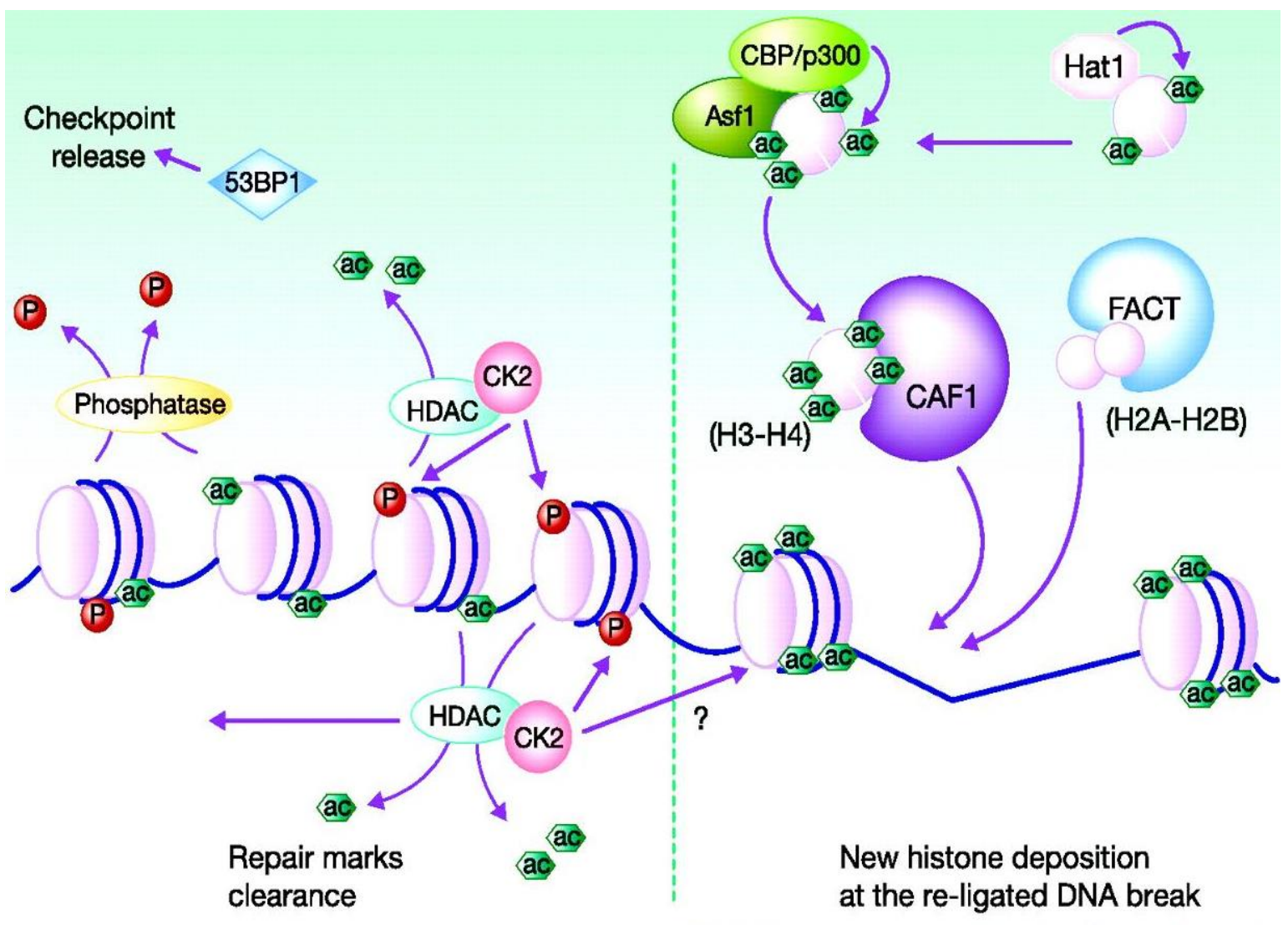

Fig 5. Chromatin restoration after DNA break repair. Upon completion of repair of the DSBs, the chromatin needs to be restored, and the repair-specific histone marks need to be removed in order to release repair factors and cell-cycle checkpoints. Thus, $\mathrm{y}-\mathrm{H} 2 \mathrm{AX}$ has to be removed from the repaired site. During chromatin restoration, new histones are deposited onto the DNA. Histone chaperones such as FACT and CAF1 have been implicated in this process. Moreover, $\mathrm{H} 3-\mathrm{H} 4$ histones deposited by CAF1 are first acetylated by Hat1, and then by CBP/p300/Rtt109-Asf1, as marks of new synthesized histones (Rossetto et al., 2010). 
The histone H3-H4 chaperones Asf1 (anti silencing function 1) and CAF-1 (chromatin assembly factor 1 ) were initially shown to be involved in nucleotide excision repair (Gaillard et al., 1996; Mello et al., 2002). Recent studies have uncovered the role of these chaperones in the DSB repair process. As in the replication process, Asf1 and CAF-1 are required for the incorporation of newly synthesized histones marked with H3K56 acetylation during DNA repair (Chen et al., 2008; Li et al., 2008; Ransom et al., 2010). Asf1, in association with yeast Rtt109 and human CBP/p300 or Gcn5 HAT, is essential for H3K56 acetylation (Das et al., 2009; Hyland et al., 2005; Tjeertes et al., 2009) (Figure 5). The FACT histone chaperone complex is associated with transcription, and its binding to chromatin was shown to be increased in response to DNA damage and depletion of the SUPT16H subunit of the FACT complex can compromise the DNA DSB repair efficiency (Kari et al., 2011). In vitro studies have shown that the FACT complex can exchange $\mathrm{H} 2 \mathrm{AX}-\mathrm{H} 2 \mathrm{~B}$ dimers and its activity is regulated by PARP activity (Heo et al., 2008). Another histone chaperone Chz1 appears to be involved in exchange of $\mathrm{H} 2 \mathrm{~A} . \mathrm{Z} / \mathrm{H} 2 \mathrm{~B}$ dimers for $\mathrm{H} 2 \mathrm{~A} / \mathrm{H} 2 \mathrm{~B}$ dimers to promote DNA repair process and inactivation of Chz1 leads to DNA damage sensitivity (Luk et al., 2007).

In the present study we analyzed the production of polyadenylated histone mRNAs from replication-dependent histone genes specifically Histone $\mathrm{H} 2 \mathrm{~B}$ genes, and their physiological role in the cell. Further we studied the role of $\mathrm{H} 2 \mathrm{~B}$ monoubiquitination in DNA damage response and repair. We showed that ATP-dependent chromatin remodeler CHD1 is recruited to DNA damage site and involved in the repair of DNA DSB through HR pathway. 


\subsection{Publication I}

\section{A subset of histone H2B genes produces polyadenylated mRNAs under a variety of cellular conditions}

\section{Citation}

Vijayalakshmi Kari*, Oleksandra Karpiuk*, Bettina Tieg, Malte Kriegs, Ekkehard Dikomey, Heike Krebber, Yvonne Begus-Nahrmann, Steven A. Johnsen

A subset of histone H2B genes produces polyadenylated mRNAs under a variety of cellular conditions

PLoS ONE 8(5): e63745.doi:10.1371/journal.pone.0063745

\section{Own contribution}

Preparation and accomplishment of the biological experiments presented in Fig 1.2, Fig I.3, Fig I.4, Fig I.5, Fig I.6, Fig I.9, Supp Fig I. S1 and Supp I. S2. i.e cell culture, inhibitor treatment, gene expression analysis, polyribosome purification, radiation treatment. Complete figure layout, tables, writing manuscript. 


\section{Abstract}

Unlike other metazoan mRNAs, replication-dependent histone gene transcripts are not polyadenylated but instead have a conserved stem-loop structure at their $3^{\prime}$ end. Our previous work has shown that under certain conditions replication-dependent histone genes can produce alternative transcripts that are polyadenylated at the $3^{\prime}$ end and, in some cases, spliced. A number of microarray studies examining the expression of polyadenylated mRNAs identified changes in the levels of histone transcripts e.g. during differentiation and tumorigenesis. However, it remains unknown which histone genes produce polyadenylated transcripts and which conditions regulate this process. In the present study we examined the expression and polyadenylation of the human histone $\mathrm{H} 2 \mathrm{~B}$ gene complement in various cell lines. We demonstrate that $\mathrm{H} 2 \mathrm{~B}$ genes display a distinct expression pattern that is varies between different cell lines. Further we show that the fraction of polyadenylated HIST1H2BD and HIST1H2AC transcripts is increased during differentiation of human mesenchymal stem cells (hMSCs) and human fetal osteoblast (hFOB 1.19). Furthermore, we observed an increased fraction of polyadenylated transcripts produced from the histone genes in cells following ionizing radiation. Finally, we show that polyadenylated transcripts are transported to the cytoplasm and found on polyribosomes. Thus, we propose that the production of polyadenylated histone mRNAs from replication-dependent histone genes is a regulated process induced under specific cellular circumstances.

\section{Introduction}

Histones are the major protein component of the eukaryotic chromatin and the transcription of the histone genes is tightly regulated. Histone mRNA levels increase up to 35 fold during the $\mathrm{S}$ phase of the cell cycle compared to the $\mathrm{G} 1$ phase and back to the basal expression level at the end of the S phase (Osley, 1991). Unlike the majority of proteincoding mRNAs, replication-dependent histone mRNAs are not spliced and lack polyA tails. Instead their $3^{\prime}$ end contains a highly conserved 16 nucleotide stem-loop sequence and a histone downstream element (HDE) which is recognized by the stem-loop binding protein (SLBP) and U7 snRNPs respectively (Marzluff et al., 2008). In addition to facilitating histone mRNA $3^{\prime}$ end processing, SLBP also facilitates their transport to cytoplasm and stimulates their degradation at the end of the $\mathrm{S}$ phase. In some cases, non-replication dependent histone variants such as $\mathrm{H} 3.3, \mathrm{H} 2 \mathrm{~A} . \mathrm{X}$ and others are expressed throughout the cell cycle, 
often in a cell type-specific manner, and display the $3^{\prime}$ end polyadenylation instead of a stem loop (Talbert and Henikoff, 2010). Studies from our lab and others have shown that the loss of correct $3^{\prime}$ end processing can result in the production of polyadenylated (poly ${ }^{+}$) histone transcripts from replication-dependent histone genes (Kirsh et al., 1989; Pirngruber et al., 2009a, 2009b; Sullivan et al., 2009; Tan et al., 2013). Depletion of various proteins including Cyclin Dependent Kinase 9 (CDK9), RING finger protein 20 (RNF20), RNF40, Nuclear Protein, Ataxia-Telangiectasia Locus (NPAT/p220), Negative Elongation Factor-E (NELF-E), members of the Cap Binding Complex $(C B C)$, or SLBP itself results in the production of poly $^{+}$histone transcripts from replication-dependent histone genes (Narita et al., 2007; Pirngruber et al., 2009a, 2009b; Sullivan et al., 2009). Importantly, several studies indicate that polyA ${ }^{+}$histone $m R N A$ levels may increase during various cellular processes including $G 1$ arrest caused by p53 accumulation (Pirngruber and Johnsen, 2010) as well as during differentiation and tumorigenesis (Abba et al., 2005; Collart et al., 1991; Kirsh et al., 1989; Martinez et al., 2007; Zhao et al., 2004). Finally, up-regulation of polyadenylated histone transcripts can be stimulated by chemical agents such as hydroxyurea (HU) (Pirngruber and Johnsen, 2010).

Despite a number studies reporting the production of polyadenylated histone mRNAs, the functional relevance of these transcripts remains unclear. It remains unknown which of the replication-dependent histone genes can give rise to polyA ${ }^{+}$transcripts. Furthermore, although it has been reported that polyadenylated histone transcripts produced following SLBP knockdown accumulate in the nucleus (Sullivan et al., 2009), it is unclear whether the poly $\mathrm{A}^{+}$histone transcripts produced from the normally replicationdependent genes under normal cellular conditions are exported to the cytoplasm and are actually translated into proteins.

In this study we examined the expression profiles of poly ${ }^{+}$and total histone transcripts produced from the entire repertoire of $\mathrm{H} 2 \mathrm{~B}$ genes and compared these in proliferating and differentiated as well as in primary normal breast and breast cancer tissues. We report that a subset of histone $\mathrm{H} 2 \mathrm{~B}$ genes also produces polyadenylated mRNA transcripts. Importantly, we also show that polyadenylated mRNA transcripts of $\mathrm{H} 2 \mathrm{~B}$ (HIST1H2BD) as well as H2A (HIST1H2AC) are transported to the cytoplasm where they are also found in the polyribosomal complexes. Importantly, we also show that levels of the 
poly $^{+}$histone transcripts increase during cellular differentiation as well as following the induction of double-strand DNA breaks via gamma-irradiation. Thus, we provide the first evidence that alternative $3^{\prime}$ end processing of histone mRNA transcripts is regulated under specific conditions and that these may lead to functional protein products.

\section{Results}

\section{Expression of replication-dependent histone H2B gene transcripts in different cell lines}

The metazoan core histone genes are clustered together in the genome. In mammals, there are two major histone gene clusters on chromosome 6p21-p22 (HIST1) and 1q21 (HIST2) as well as one minor cluster on 1q42 (HIST3) (Marzluff et al., 2002). Each of the histone proteins is encoded by several histone genes and there are 18 histone H2B genes reported for human. To date it is unclear to what extent each of the individual histone genes are actually expressed, and whether this expression varies between tissues, cell types or under different physiological conditions. Since the expression levels of the various $\mathrm{H} 2 \mathrm{~B}$ genes remain largely unknown, we examined the expression levels of replication-dependent H2B transcripts in different cell lines including H1299, MCF7, MCF10A, U2OS and hMSCs via real time quantitative PCR (qRT-PCR) (Fig I.1 A-E). The expression of individual H2B gene transcripts was represented as relative genomic DNA units (Rel. gDNA) as described in materials and methods to enable the quantitative comparison between different genes. For each cell line tested we observed distinct $\mathrm{H} 2 \mathrm{~B}$ gene expression profiles. While many genes were either consistently expressed at medium to high levels (HIST1H2BC, HIST1H2BD, HIST1H2BE, HIST1H2BJ, HIST1H2BK, HIST1H2BL, HIST1H2BM and HIST1H2BN) and others were very low or undetectable in all cell lines tested (HIST1H2BA, HIST1H2BB, HIST2H2BF and $H I S T 2 H 2 B B$ ) other genes displayed cell line-specific expression. For example, while HIST1H2BM is expressed at medium levels in H1299, MCF10A, U2OS and hMSC cells, it represents one of the major expressed H2B genes in MCF7 cells. Similarly, while HIST2H2BE expression was nearly undetectable in H1299, MCF10A and U2OS cell lines, moderate expression was observed in MCF7 and hMSCs. The HIST1H2BG and HIST1H2BI genes also showed cell line-specific expression in which they were moderately expressed in H1299, MCF7 and hMSCs, but very low in U2OS and MCF10A cells. HIST1H2BF was also broadly 
expressed in the cell lines except in MCF10A where expression was very low. Thus, the repertoire of $\mathrm{H} 2 \mathrm{~B}$ genes expressed appears to be regulated in a cell context-specific manner.

A

H1299

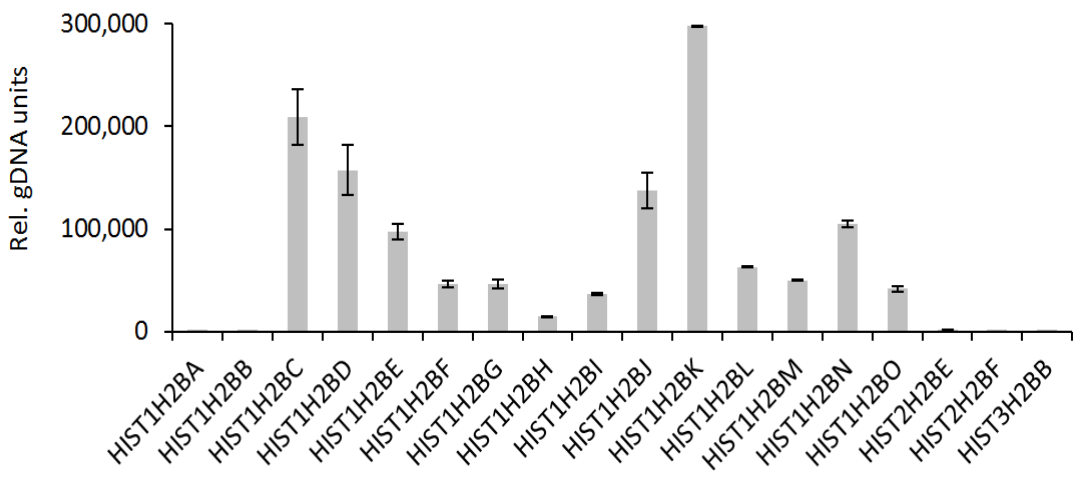

B MCF7

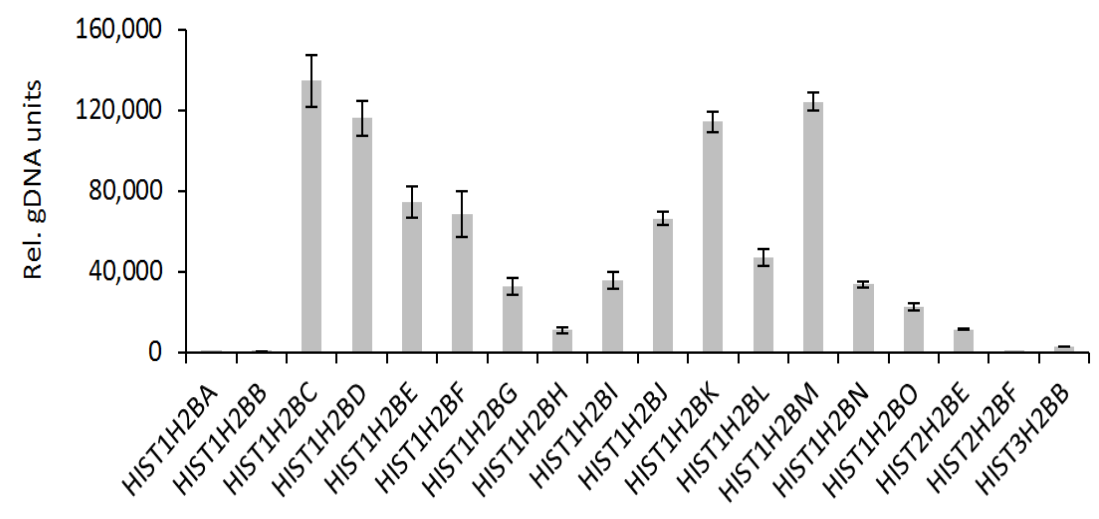

C

\section{MCF10A}

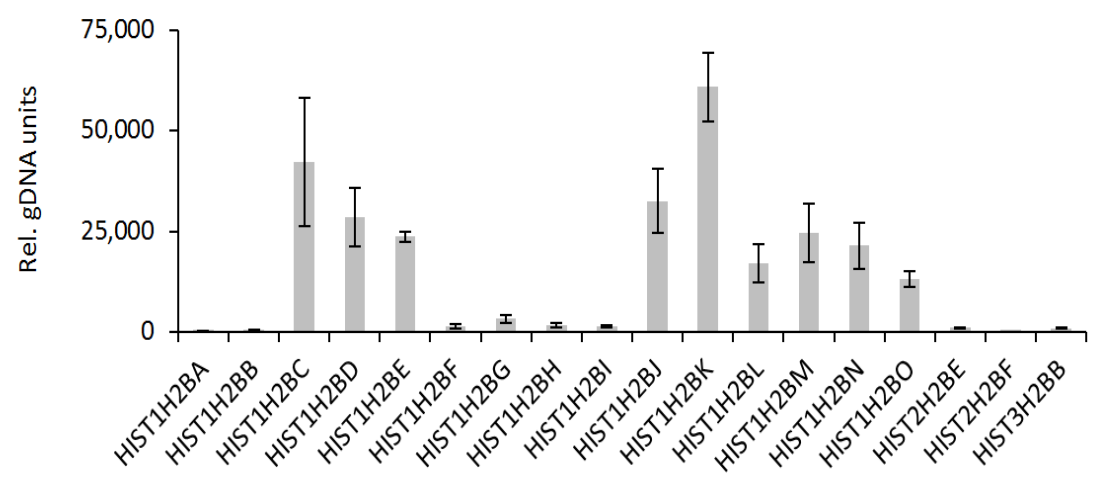


D

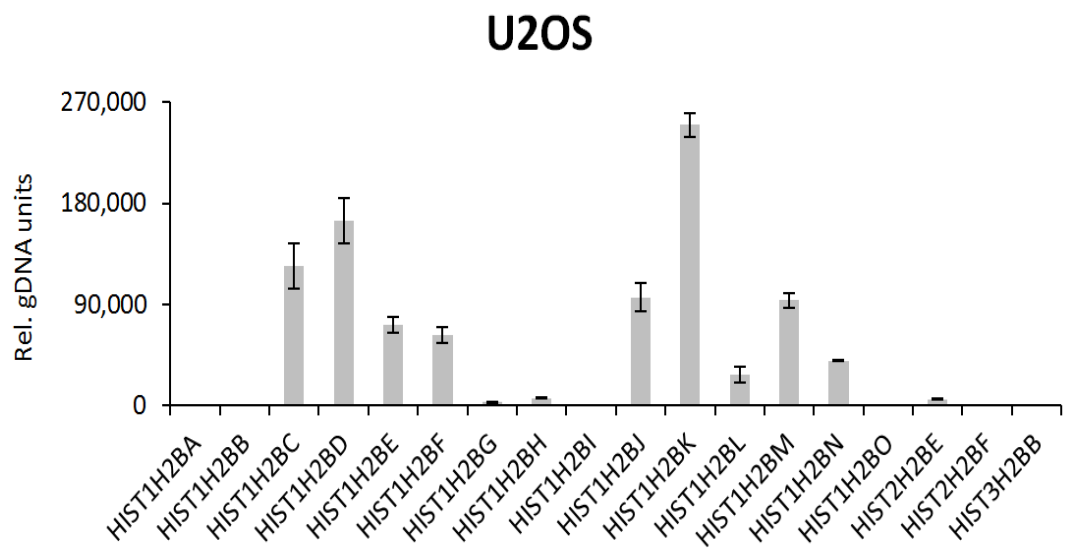

$E$

\section{hMSCs}

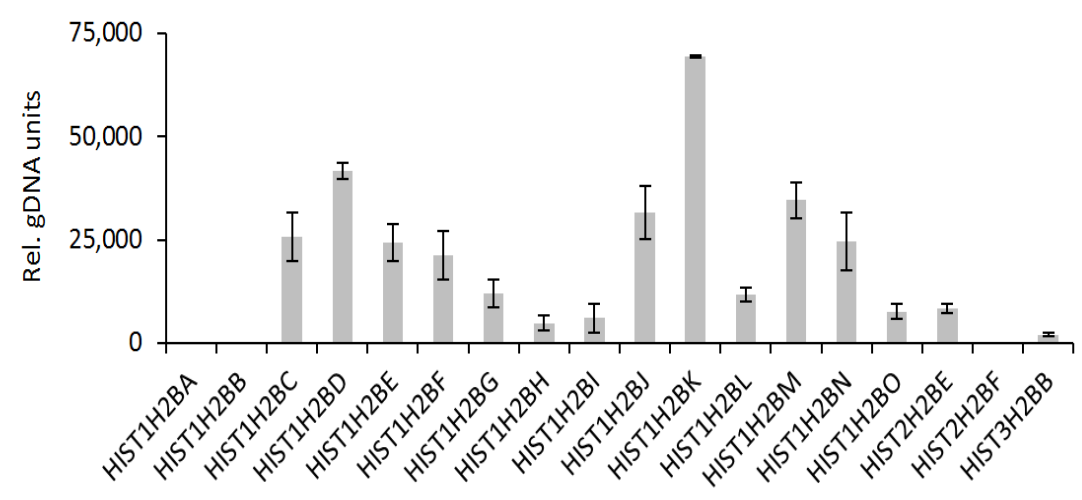

Fig I.1: Expression of the histone $\mathrm{H} 2 \mathrm{~B}$ gene complement in different cell lines. Expression of different $\mathrm{H} 2 \mathrm{~B}$ genes in the indicated cell lines was analyzed by qRT-PCR. Relative expression values between the individual genes were normalized using diploid genomic DNA (see materials and methods) and indicated as "Rel. gDNA units". Mean \pm SD, $n=3$.

\section{H2B mRNAs are differentially polyadenylated upon p53-induced cell cycle arrest}

In our previous studies, we demonstrated that the manipulation of epigenetic regulatory pathways (Pirngruber et al., 2009a, 2009b) or the induction of a G1 cell cycle arrest (Pirngruber and Johnsen, 2010) results in an increase in the production of spliced and polyadenylated transcripts from the HIST1H2BD and HIST1H2AC genes. Thus, after comparing the overall expression levels of different histone genes in various cell lines, we next examined which of them give rise to polyA ${ }^{+}$transcripts. In order to do this, we purified total and polyA ${ }^{+}$mRNA from HCT116 cells 
A

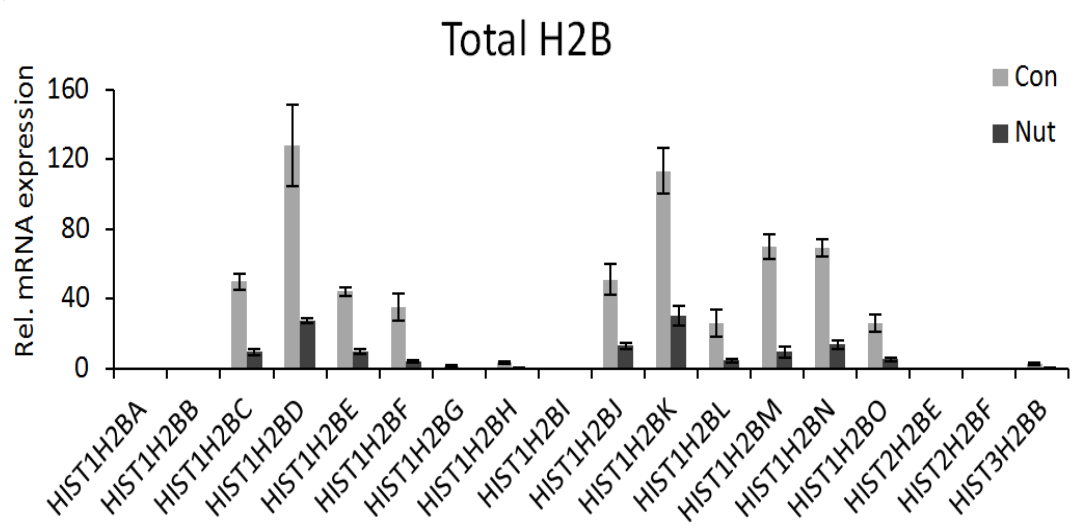

B

Polyadenylated H2B

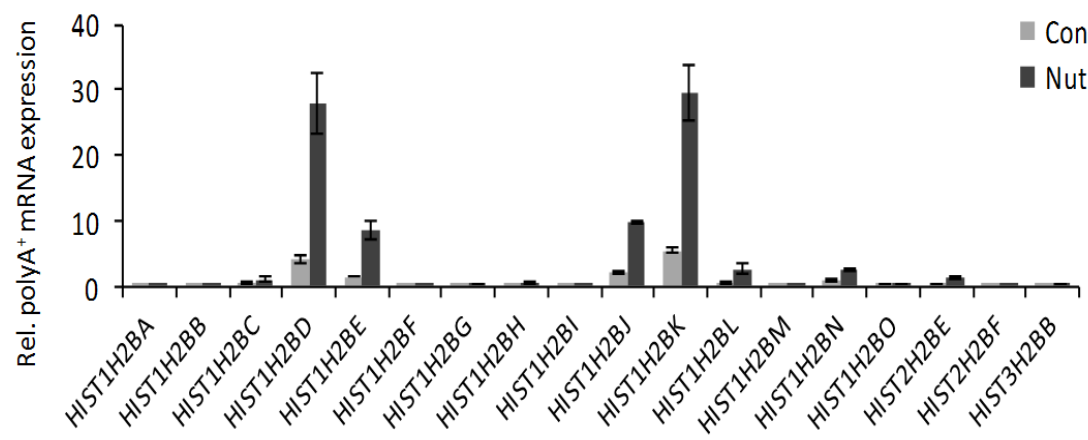

C

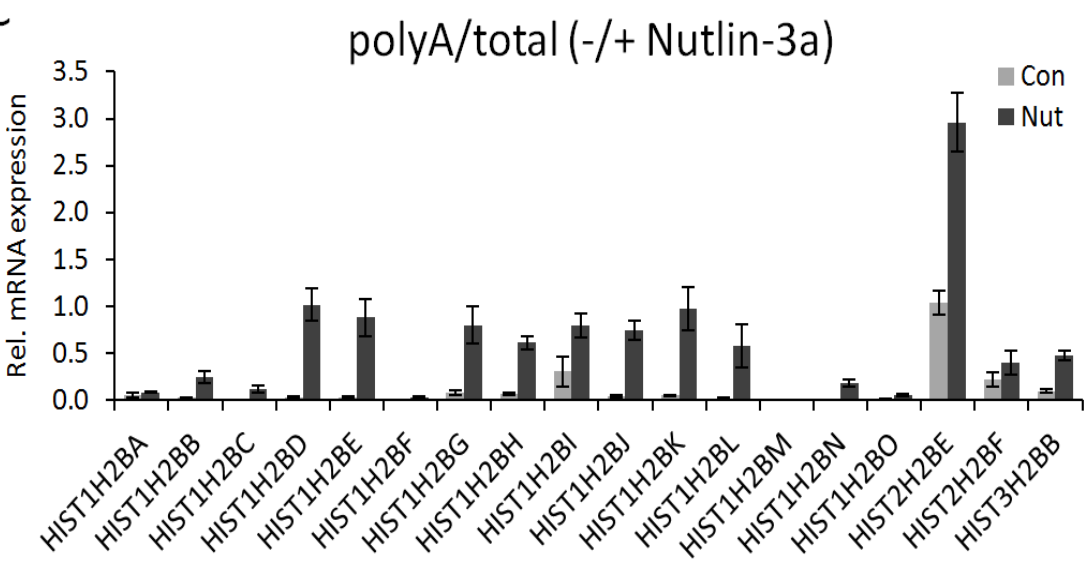

Fig I.2: Nutlin-3a treatment down-regulates the expression of normal replication-dependent histone H2B genes and up-regulates the expression polyA+ transcripts. (A) Total expression of different replication-dependent histone H2B genes in control and Nutlin-3a treated HCT116 cells. Total RNA was reverse transcribed using random primers and analyzed by qRT-PCR for H2B genes as in Fig. 1. Mean $\pm S D, n=3$. (B) Expression of polyA+ histone H2B transcripts in HCT116 cells upon 
Nutlin-3a treatment. PolyA+ mRNA was purified as described in materials and methods and reverse transcribed using random primers. Transcript levels of polyA+ H2B genes were analyzed by qRT-PCR. Mean $\pm S D, n=3$. (C) Levels of polyA+H2B transcripts normalized to the total H2B levels from (B). Mean $\pm S D, n=3$.

treated for 24 hours with Nutlin-3a, a small molecule inhibitor of the p53 ubiquitin ligase MDM2 (Vassilev et al., 2004) which induces a G1 cell cycle arrest in p53-proficient HCT116 cells (Pirngruber and Johnsen, 2010), and examined the expression of each of the H2B genes via qRT-PCR analysis. To validate the purity of polyA ${ }^{+}$mRNA purified from control and Nutlin-3a treated cells, we analyzed for the presence of ribosomal rRNA transcripts (5.8S rRNA and 18S rRNA) which are not polyadenylated (Fig I. S1A). Consistent with the earlier reports Nutlin-3a treatment decreased the overall expression of all detectable $\mathrm{H} 2 \mathrm{~B}$ transcripts (irrespective of polyadenylation status) (Fig I.2A). Interestingly, many H2B genes demonstrated a significant increase in the amount of poly ${ }^{+}$transcript production following Nutlin-3a treatment (Fig I.2B).

Notably, the HIST1H2BD, HIST1H2BE, HIST1H2BJ, HIST1H2BK genes were highly expressed and also showed a significant increase in the fraction of polyadenylated transcripts (Fig I.2B, 2C). Normalization of poly $A^{+} \mathrm{H} 2 \mathrm{~B}$ mRNA levels to total H2B expression revealed that the fraction of polyadenylated transcripts is similarly up-regulated upon Nutlin-3a treatment for several genes irrespective of their overall expression levels. For example, the levels of polyadenylated transcripts from the HISTIH2BG, HIST1H2BH, and $\mathrm{HIST} 1 \mathrm{H} 2 \mathrm{BI}$ genes are upregulated to a similar extent as the more highly expressed genes HIST1H2BD and HIST1H2BK (Fig I.2C). Importantly, not all transcribed H2B genes demonstrated these effects. For example, HIST1H2BC, HSIT1H2BF, HIST1H2BM and $\mathrm{HIST} 1 \mathrm{H} 2 \mathrm{BO}$ are all expressed at significant levels, but show only very little or no evidence of poly $^{+}$transcripts (Fig I.2). Thus the production of polyadenylated mRNAs from histone $\mathrm{H} 2 \mathrm{~B}$ genes is regulated in a gene-specific manner. Recent studies using a transcriptome-wide direct RNA sequencing (DRS) approach enable the precise mapping and quantification of polyadenylation sites as well as the identification of differentially polyadenylated RNA transcripts (Lin Y, Ozsolak F). We used the recently developed XPAD server genome browser (http://johnlab.org/xpad/) to map the DRS reads on histone H2B genes for the breast cancer cell line MCF7 (Fig I.3A), as well as the normal mammary epithelial cell line MCF10A (Fig I.3B). Consistent with the data in HCT116 cells, the mapping of DRS reads demonstrated that 
A

MCF7

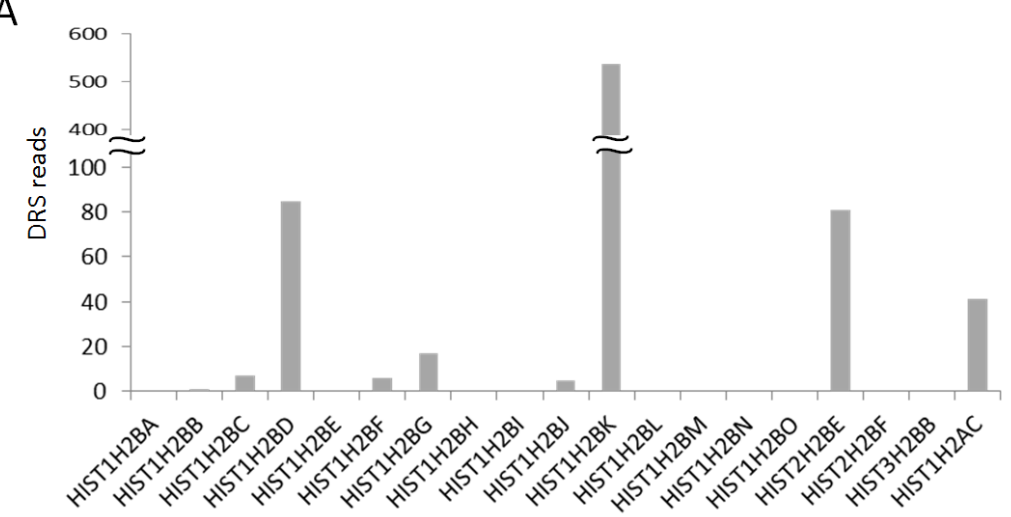

B

MCF10A

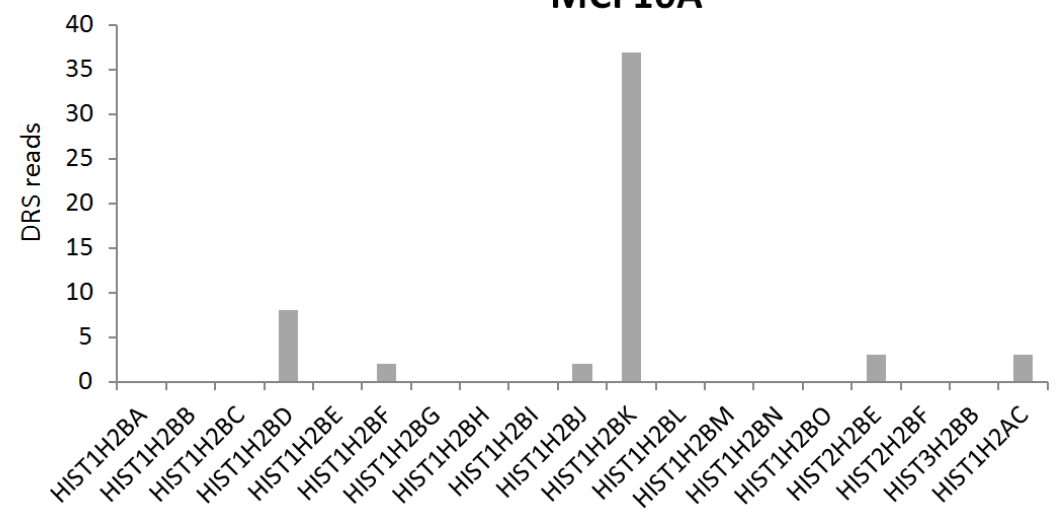

C

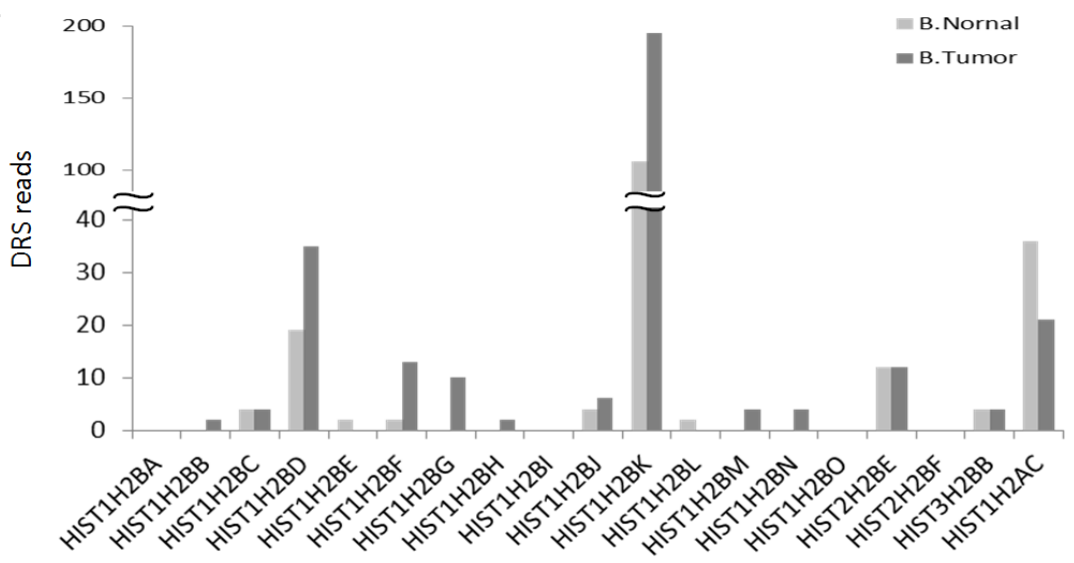

Fig I.3: Polyadenylation of histone H2B genes assigned using polyadenylation and alternative polyadenylation (APA) map. Number of reads at polyA sites on different replication- dependent histone H2B genes which are mapped using XPAD server (http://johnlab.org/xpad/) in (A) MCF7 (human breast cancer cell line), (B) MCF10A (immortalized human mammary epithelial cell line) cells and (C) normal and breast tumor tissues. 
only a subset of $\mathrm{H} 2 \mathrm{~B}$ genes has detectable polyadenylation sites in MCF7 and MCF10A cells (Fig I.3, Supplementary Fig I.S3). Moreover, H2B transcripts identified as being highly polyadenylated in HCT116 cells (e.g., HIST1H2BD and HIST1H2BK; Fig I.2B) were also found to possess polyadenylation sites in MCF7 and MCF10A cells. Interestingly, using a further set of DRS mapping data, we observed that the number of reads identified for poly ${ }^{+}$histone transcripts increased in tumor breast samples compared to normal breast epithelium (Fig I.3C) possibly suggesting that increased levels of polyA ${ }^{+}$histone transcripts may provide an advantage to tumor cells.

\section{Polyadenylated histone H2B transcripts are transported to the cytoplasm and found on polyribosomes}

Metazoan replication-dependent histone mRNAs are single exonic and are not spliced. Importantly, the inclusion of an intron prevents proper stem loop-dependent mRNA $3^{\prime}$ end processing suggesting, that stem loop-directed $3^{\prime}$ end processing of histone mRNAs is mutually exclusive with splicing and polyadenylation (Pandey et al., 1990). We have previously shown that some histone genes (e.g. HIST1H2BD and HIST1H2AC) produce both canonically processed replication-dependent mRNAs as well as longer, spliced replicationindependent mRNAs produced using a downstream second exon (Pirngruber and Johnsen, 2010; Pirngruber et al., 2009a). Due to the size of the primary transcript and the distance between the canonical $3^{\prime}$ end processing site and the polyadenylation site, these two transcripts can more easily be distinguished from their non-polyadenylated counterparts than transcripts produced from polyadenylation sites located immediately downstream of the canonical stem loop-directed $3^{\prime}$ end processing site (e.g., HIST1H2AA).

Thus, we verified the expression of total and polyadenylated HIST1H2BD and HIST1H2AC transcripts in HCT116 cells arrested in G1 phase by Nutlin-3a treatment (Fig I.4A). Consistent with our previous results, Nutlin-3a treatment increased the levels of polyA $^{+}$HIST1H2BD and HIST1H2AC transcripts while decreasing their overall levels (i.e., canonically processed and polyadenylated together). These results were further verified in polyA ${ }^{+}$purified mRNA (Fig I.4B). 
A
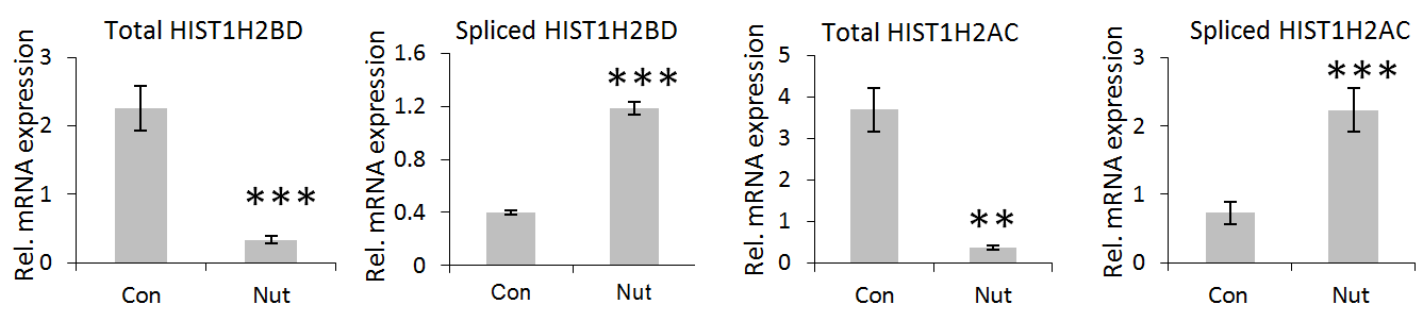

B
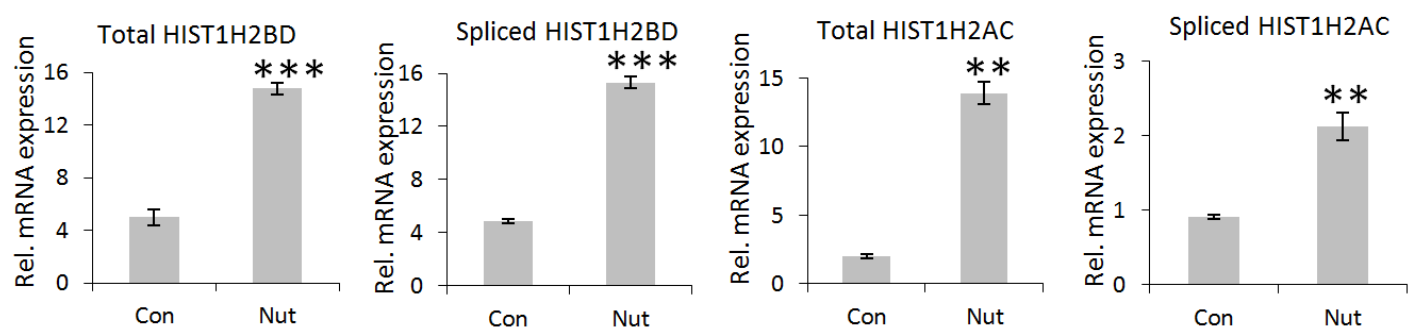

Fig I.4: Expression of normal and PolyA ${ }^{+}$HIST1H2BD and HIST1H2AC transcripts in HCT116 cells. (A) Cells were treated with Nutlin-3a as in Fig. 2. RNA was reverse transcribed into cDNA using both random and poly-T primers to check the mRNA levels of HIST1H2BD and HIST1H2AC, total and polyadenylated transcripts respectively. Values were normalized to $R P L P O$ expression. Mean $\pm \mathrm{SD}, \mathrm{n}=$ 3. (B) Enrichment for polyadenylated histone transcripts using PolyATtract ${ }^{\circledR}$ mRNA Isolation System III. Total RNA was used to isolate polyadenylated RNA and reverse transcribed using poly-T primers. Expression of total and polyA ${ }^{+} H I S T 1 H 2 B D$ and HIST1H2AC transcripts was analyzed by qRT-PCR. Values were normalized to RPLPO expression. Mean $\pm \mathrm{SD}, \mathrm{n}=3$. P-values were calculated and statistical significance was represented as follows (** $P \leq 0.01 ; * * * P \leq 0.001)$.

Although a number studies examined the "expression" of polyA ${ }^{+}$histone transcripts (primarily through microarray analysis), whether or not these transcripts are actually exported from the nucleus and translated was unclear. We hypothesized that poly $A^{+}$histone mRNAs may be translated and give rise to proteins. To examine whether polyA ${ }^{+}$histone mRNA is transported to the cytoplasm we isolated cytoplasmic RNA from HCT116 cells and examined it for the presence of spliced and polyadenylated histone HIST1H2BD and HIST1H2AC mRNA. qRT-PCR analysis with the cytoplasmic RNA confirmed the presence of HIST1H2BD and HIST1H2AC spliced transcripts, indicating that polyA ${ }^{+}$histone mRNAs are indeed transported to the cytoplasm (Fig I.5A). To further determine whether these polyA ${ }^{+}$ histone mRNA transcripts are actually translated, we isolated polyribosomes from control and Nutlin-3a treated HCT116 cells. The representative polyribosome profiles are shown in Fig I.5B. qRT-PCR analyses of polyribosome-bound RNA clearly demonstrated the presence of poly $\mathrm{A}^{+}$histone gene transcripts, supporting the conclusion that polyA+ transcripts may 
give rise to proteins and thereby contribute to the maintenance of histone protein levels. Furthermore, we also observed a Nutlin-3a-induced increase of the polyribosome-bound polyA+ fraction vs. a decrease in polyribosome-bound total histone mRNA levels (Fig I.5C).

A
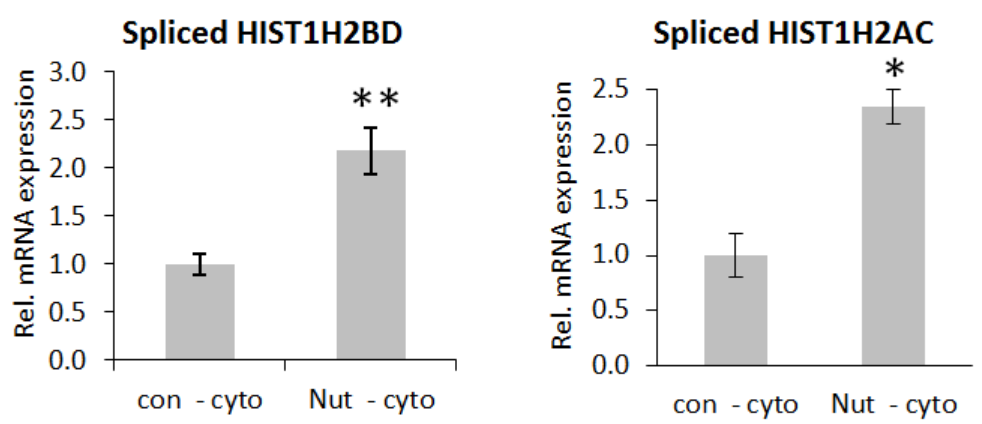

B

Control - 1
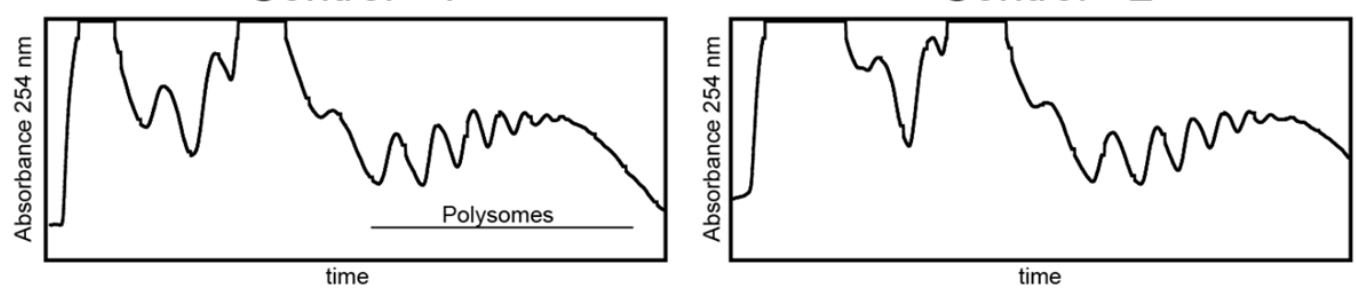

Nutlin - 1

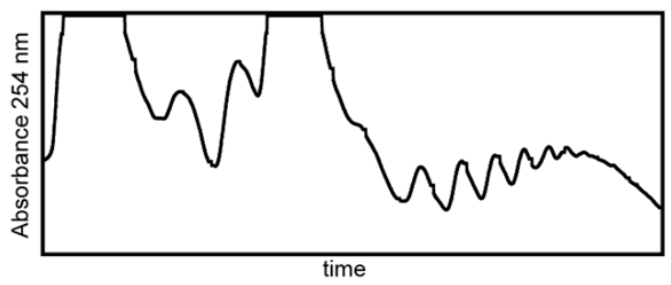

Nutlin - 2

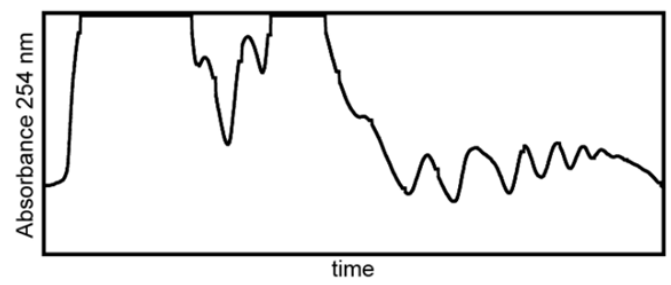

C
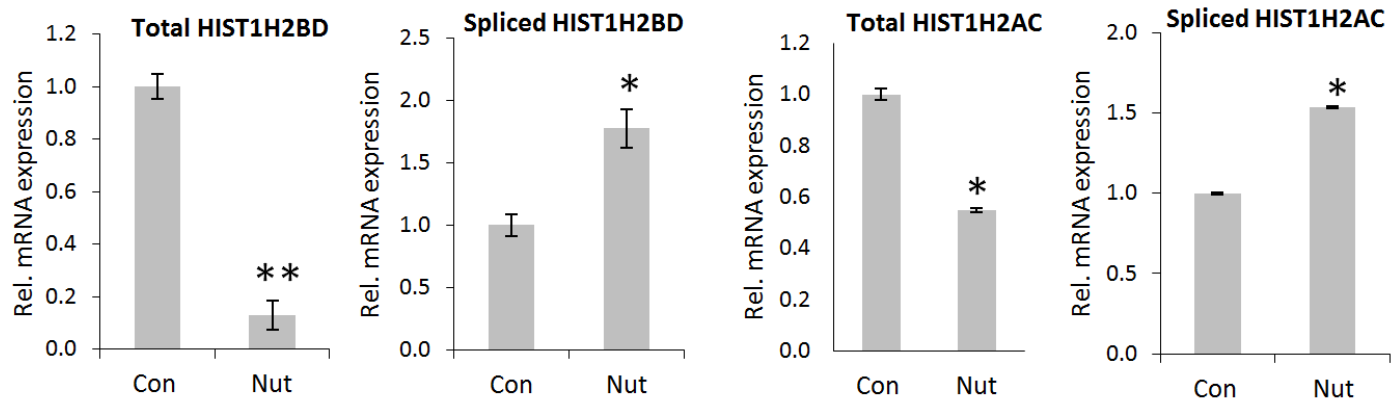
Fig I.5: Spliced HIST1H2BD and HIST1H2AC transcripts are transported to the cytoplasm and found on polyribosomes. (A) Polyadenylated HIST1H2BD and HIST1H2AC mRNA is transported to the cytoplasm. Cytoplasmic RNA was isolated from HCT116 cells treated either with DMSO or Nutlin-3a for 24 hours. RNA was reverse transcribed using random primers and analyzed for spliced HIST1H2BD and HIST1H2AC transcript by qRT-PCR. Values were normalized to $18 \mathrm{~S}$ rRNA. Mean \pm SD, $\mathrm{n}=3$. (B) Representative polyribosome profiles obtained after sucrose gradient fractionation from DMSO and Nutlin-3a treated HCT116 cells. The $x$-axis represents the time of elution and $y$-axis represents the absorbance at $254 \mathrm{~nm}$, indicating the RNA content. Polysome profiles were indicated in the figure. (C) RNA was extracted from the indicated polyribosome fractions of DMSO and Nutlin3a treated cells and reverse transcribed using random primers. Expression of total and spliced HIST1H2BD and HIST1H2AC mRNA was analyzed by qRT-PCR and values were normalized to $18 \mathrm{~S}$ rRNA. Mean $\pm S D, n=2$. $P$-values were calculated and statistical significance is represented as follows (*P $\leq 0.05 ; *$ P $\leq 0.01)$.

\section{Radiation-induced expression of poly ${ }^{+}$gene transcripts}

After establishing that poly $A^{+}$transcripts can be transported to cytoplasm and translated, we investigated whether the levels of polyA ${ }^{+}$histone transcripts may be regulated under physiological circumstances. Initially, we tested whether exposure of A549 lung carcinoma cells to $\mathrm{y}$-radiation $\left(6 \mathrm{~Gy}\right.$ ) affects the levels of poly ${ }^{+}$histone mRNAs. Consistent with the effects of Nutlin-3a-induced cell cycle arrest, $24 \mathrm{~h}$ after irradiation the mRNA levels of spliced HIST1H2BD and HIST1H2AC (Fig I.6A) were significant elevated despite an overall decrease in total histone transcript levels (Fig I.6B).

\section{PolyA $^{+}$histone mRNA expression is up-regulated during cellular differentiation}

We previously hypothesized that terminal cellular differentiation may result in changes in histone mRNA polyadenylation (Pirngruber and Johnsen, 2010). To test this hypothesis, we utilized an immortalized human mesenchymal stem cell (hMSC) line which can be differentiated to the osteoblast, adipocyte or chondrocyte lineages (Simonsen et al., 2002). We differentiated hMSCs into either adipocytes or osteoblasts for 5, 10 or 15 days and confirmed the expression of differentiation-specific genes PPARG (Fig I.7A) for the adipocyte lineage and BGLAP for the osteoblast lineage (Fig I.7B) before analyzing the expression of spliced HIST1H2BD and HIST1H2AC transcripts Fig I.7C, D). Consistent with our hypothesis, the expression of spliced HIST1H2BD (Fig I.7C) and HIST1H2AC (Fig I.7D) mRNAs was significantly increased in differentiated adipocytes and osteoblasts compared to undifferentiated hMSCs irrespective of the differentiation lineage. 
A

Spliced HIST1H2BD

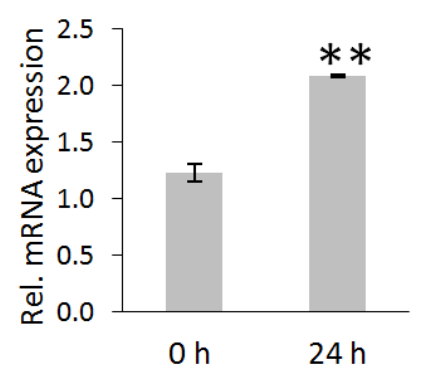

B

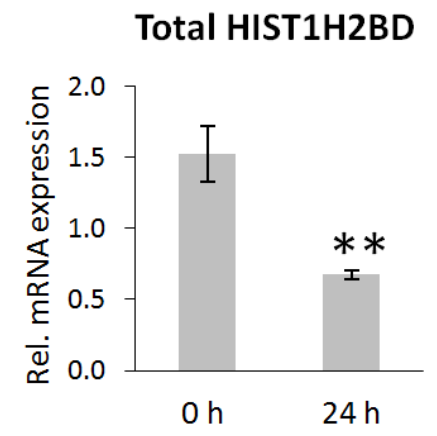

C

\section{HIST1H2BD spl/total}

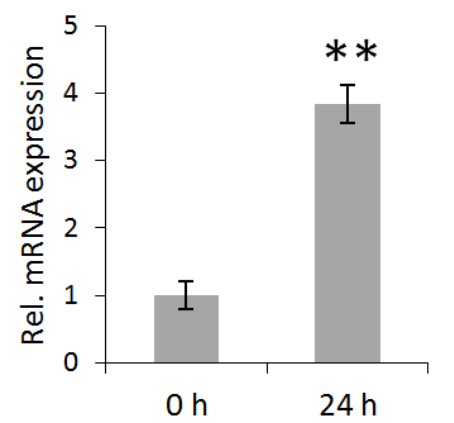

Spliced HIST1H2AC

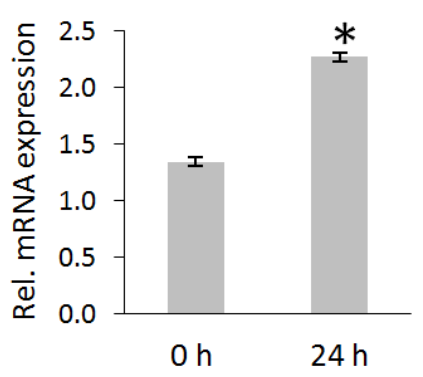

Total HIST1H2AC
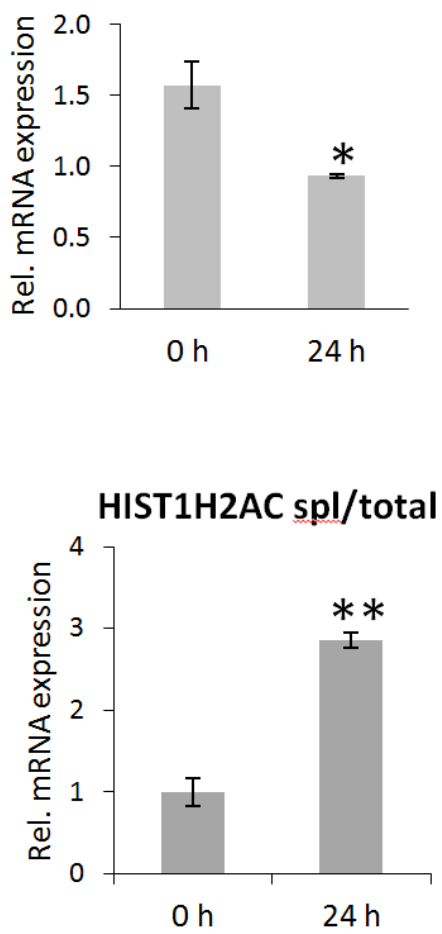

Fig I.6: Radiation induced elevated expression of spliced histone transcripts. A549 cells were exposed to gamma-irradiation ( $6 \mathrm{~Gy}$ ) and incubated for 24 hours. RNA was extracted and analyzed by qRT-PCR for (A) spliced and (B) total HIST1H2BD and HIS1H2AC. Values were normalized to RPLPO. Mean $\pm S D, n=3$. (C) Expression of spliced HIST1H2BD and HIST1H2AC transcripts was normalized to the total HIST1H2BD and HIST1H2AC levels. P-values were calculated and statistical significance is represented as follows ( $\left.{ }^{*} \mathrm{P} \leq 0.05 ;{ }^{* *}-\mathrm{P} \leq 0.01\right)$. 

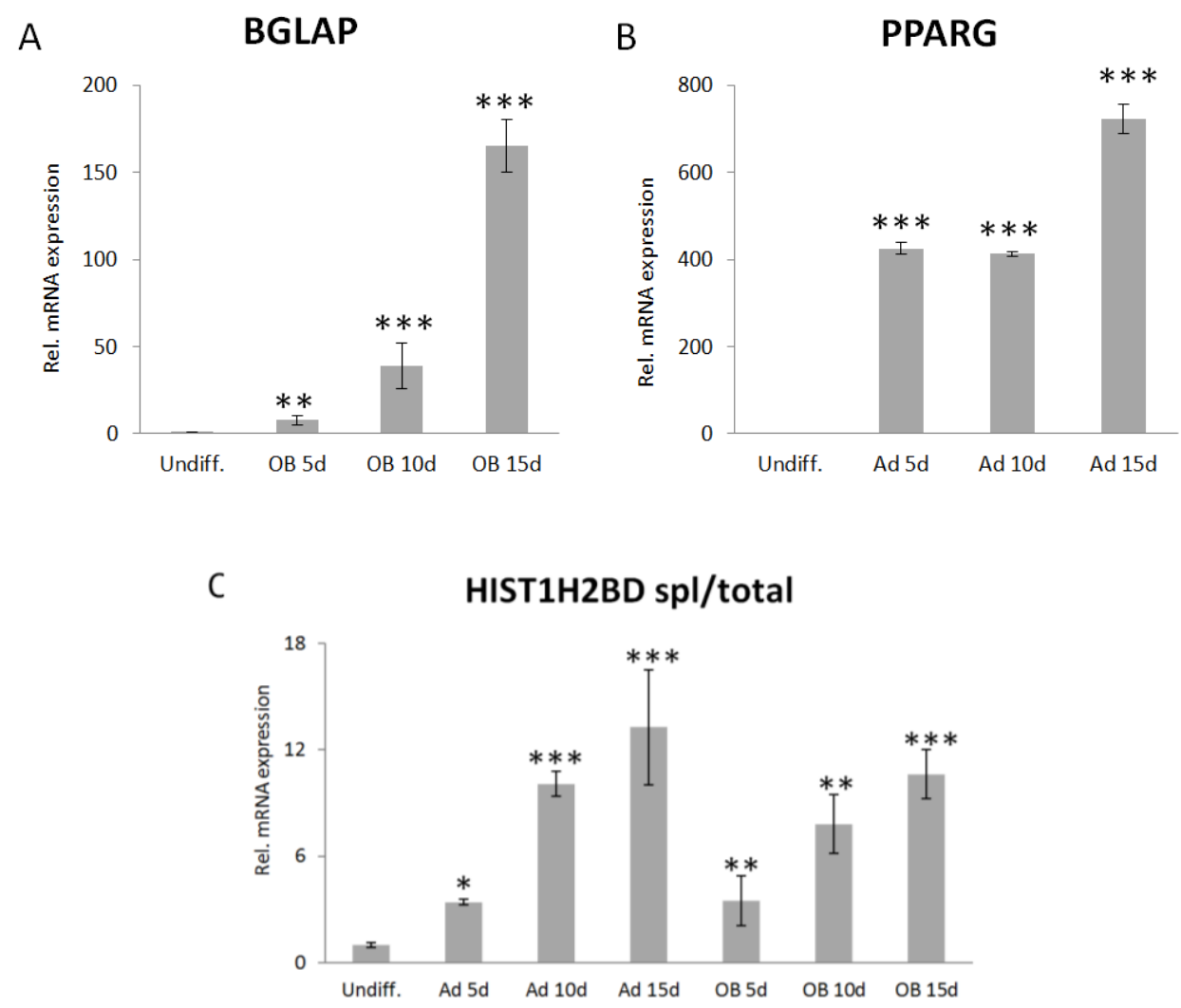

D

\section{HIST1H2AC spl/total}

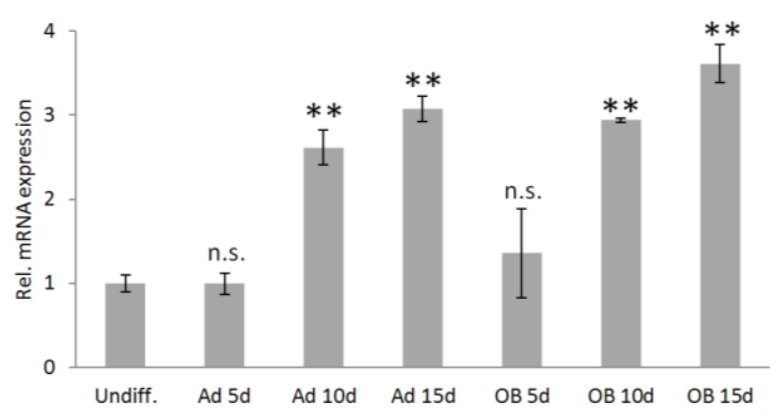

Fig I.7: Differentiation of uncommitted mesenchymal stem cells results in elevated expression of spliced up to histone transcripts. (A, B) hMSCs were differentiated into (A) adipocytes or (B) osteoblasts for 15 days. Expression of marker genes PPARG for adipocytes and BGLAP for osteoblasts was analyzed by qRT-PCR. Values were normalized to HNRNPK expression. Mean $\pm S D, n=3$. (C, D) The expression of (C) spliced HIST1H2BD or (D) spliced HIST1H2AC was analyzed by qRT-PCR using same samples as in (A) and (B). To obtain relative amounts of spliced transcript its expression was normalized to (C) total HIST1H2BD or (D) HIST1H2AC expression. Mean \pm SD, $\mathrm{n}=3$. P-values were calculated and statistical significance is represented as follows ( $\mathrm{ns} P>0.05 ;{ }^{*} \mathrm{P} \leq 0.05$; ${ }^{* *} \mathrm{P} \leq 0.01$; $* * * \mathrm{P} \leq 0.001)$. 

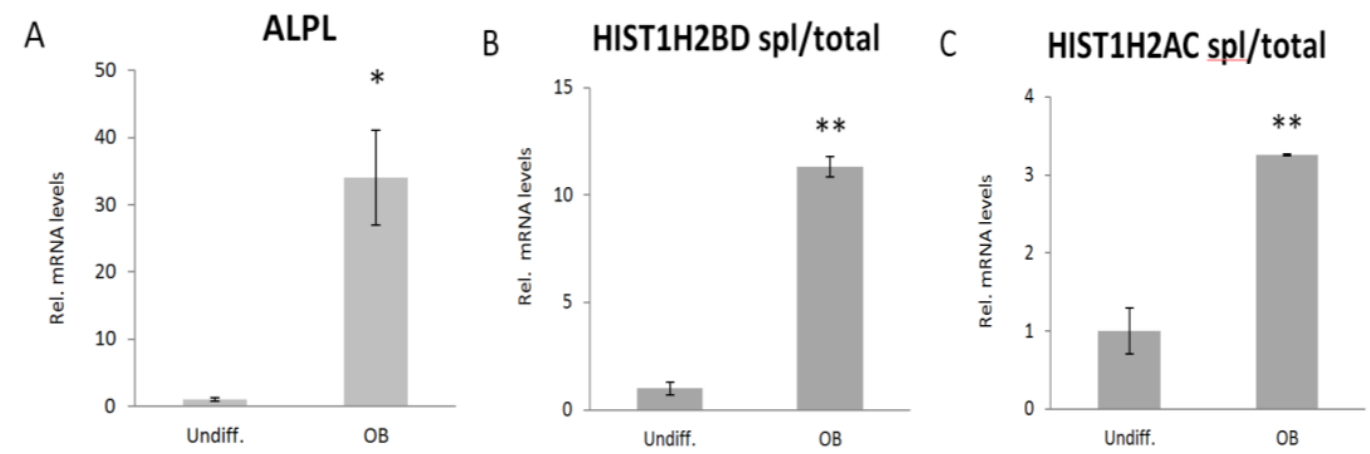

Fig 1.8: Expression of spliced histone transcripts are increased during committed osteoblast differentiation. (A) hFOB 1.19 cells were differentiated into osteoblasts for 7 days. Alkaline phosphatase ( $A L P L$ ) expression in undifferentiated (undiff.) and differentiated $(O B)$ cells was analyzed by qRT-PCR. Values were normalized to HNRNPK expression. Mean $\pm S D, n=3$. $(B, C)$ Samples shown in (A) were examined for (B) spliced HIST1H2BD and (C) HIST1H2AC expression. Values were normalized to total HISTH2BD and HISTH2AC respectively. Mean $\pm S D, n=3$. P-values were calculated and statistical significance is represented as follows $\left({ }^{*} \mathrm{P} \leq 0.05 ;{ }^{* *} \mathrm{P} \leq 0.01\right)$.

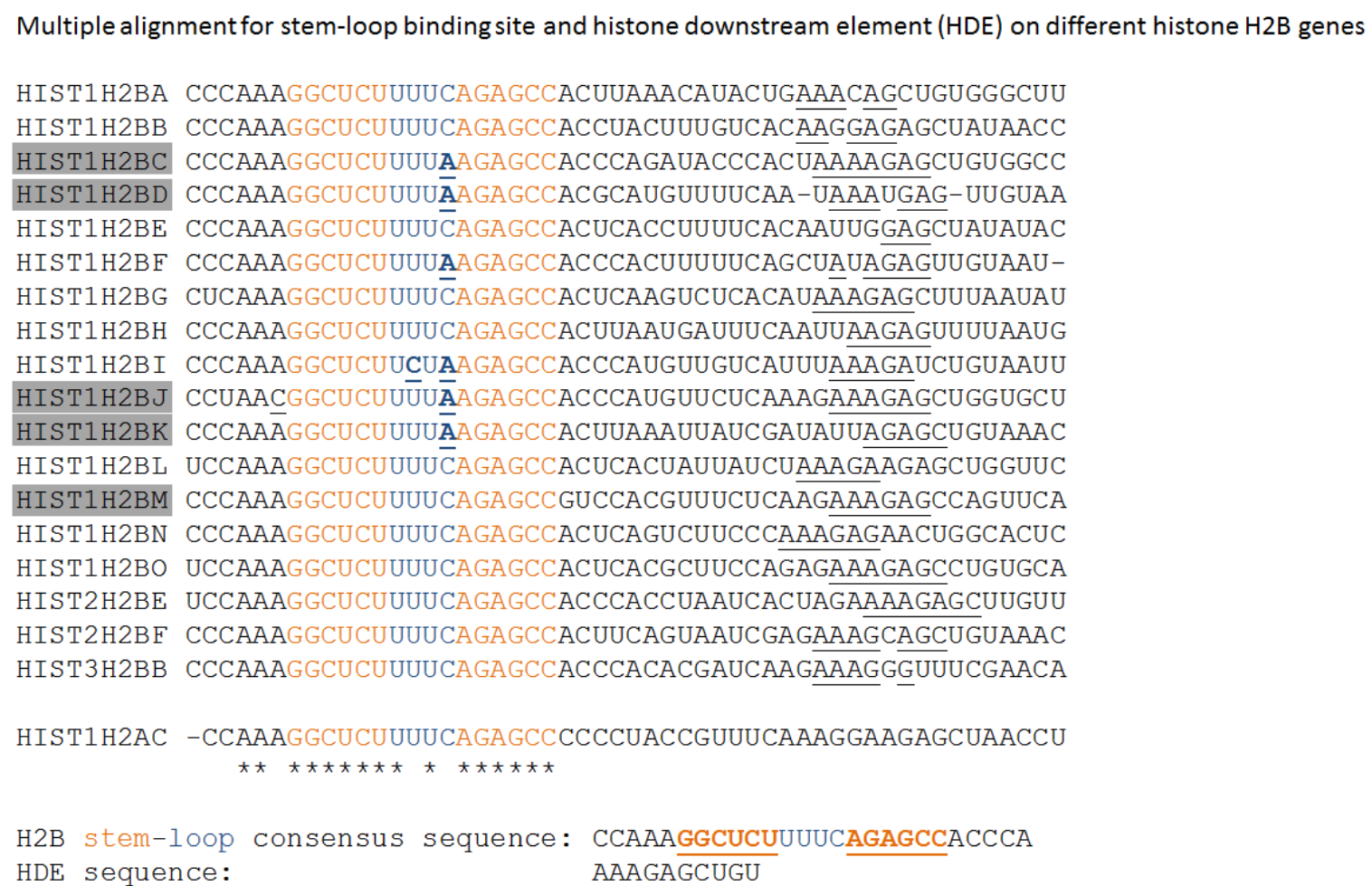

Fig 1.9: Comparison of stem-loop sequences in H2B genes. Alignment of the stem-loop sequences of H2B genes performed with ClustalW2 multiple sequence alignments tools (http://www.ebi.ac.uk/ Tools/msa/clustalw2/). Highly expressed histones are marked in grey. Stem loop sequence is shown in orange, bases that are different from canonical are underlined. 


\section{Discussion}

In proliferating metazoan cells most histone synthesis is coupled to DNA replication and occurs during the $S$ phase of the cell cycle. While the transcription of replicationdependent histone mRNAs is cell cycle regulated, the histone mRNAs themselves also have several specific features. First of all, they are not polyadenylated at their 3' ends, but instead possess a specific stem-loop structure that is recognized by a unique $3^{\prime}$ end processing machinery and aids both in the $3^{\prime}$ end cleavage as well as in nuclear export and translation. Secondly, replication-dependent histone transcripts contain only one exon and, unlike most mRNAs, are not spliced. Despite significant transcriptional regulation during $\mathrm{S}$ phase, a certain degree of basal level histone synthesis was also observed throughout the cell cycle, independently of replication (Wu and Bonner, 1981b). Since stem-loop processing is coupled to the $S$ phase, this phenomenon may be explained by mRNA processing events independent of the cell cycle. This hypothesis was supported by the identification of replication-independent histone mRNAs that are produced from the same histone genes as replication-dependent transcripts, but additionally contain a polyadenylation site downstream of their stem loop sequence (Kirsh et al., 1989; Pirngruber and Johnsen, 2010). Interestingly, poly $\mathrm{A}^{+}$histone transcripts from replication-dependent histone genes were also detected in C. elegans (Mangone et al., 2010) as well as in mouse ES cells and post mitotic neurons (Shepard et al., 2011)) via direct RNA sequencing, suggesting that poly ${ }^{+}$transcripts may have emerged early during evolution to facilitate the basal histone production.

Increased production of poly ${ }^{+}$histone transcripts was shown to be induced by a wide range of factors including depletion of epigenetic regulators, induction of DNA damage or serum starvation (Pirngruber and Johnsen, 2010; Pirngruber et al., 2009a, 2009b). Furthermore, numerous microarray-based studies have observed changes in the "expression" of replication-dependent histone mRNA transcripts during tumorigenesis and differentiation (Martinez et al., 2007; Yan et al., 2007). Given the fact that these studies were based on poly-T reverse transcription, it seems likely that histone mRNA polyadenylation is a process regulated under diverse conditions. Tumor suppressor p53 mediated cell-cycle arrest, implicated in the regulation of proliferation and tumorigenesis, also controls the expression of poly $\mathrm{A}^{+}$histone transcripts via p21-dependent cell cycle arrest (Pirngruber and Johnsen, 2010). Despite numerous studies reporting the expression of 
polyA $^{+}$histone mRNAs from the replication-dependent histones genes, the functional importance of these transcripts remains unknown and has been refuted (Sullivan et al., 2009). In this study we examined the expression of polyA ${ }^{+}$histone transcripts from replication-dependent histone genes which normally primarily produce 3'stem-loop containing mRNAs, under various cellular conditions.

First of all, using the histone $\mathrm{H} 2 \mathrm{~B}$ gene complement as a model system for our studies, we compared the expression of different H2B genes. Surprisingly, we observed a wide range of expression of the individual genes, suggesting that the regulation of histone transcription and mRNA processing is gene-specific and more complex process than may have been previously assumed. Moreover, we demonstrated that different cell lines exhibit distinct expression patterns of total and polyadenylated H2B mRNA. The efficiency of transcription might be dependent on promoter context and/or mRNA 3' UTR sequence. A pervious study demonstrated that minor changes in the stem loop sequence can significantly affect $3^{\prime}$ end processing efficiency (Pandey et al., 1990). Interestingly, a recently published study shows that the structure, rather than the sequence of the stem loop is essential for proper SLBP binding (Tan et al., 2013). Furthermore, the human 3' exonuclease ( $3^{\prime} \mathrm{hExO}$ ) involved in trimming of histone mRNAs cleaved in an SLBP-directed manner binds to specific sites within the 3' stem loop including C15 within the loop sequence. Surprisingly, a number of H2B mRNAs depart from the stem loop consensus sequence (Fig. 9). Interestingly, we observed that 4 out of 5 highly expressed H2B histone genes (HIST1H2BC, HIST1H2BD, HIST1H2BJ and HIST1H2BK) possess a single nucleotide mutation at C15 (C to A) within the loop sequence (Fig I.9). In addition, the 5 nucleotides $5^{\prime}$ to the stem loop (CCAAA) are also recognized by SLBP (Tan et al., 2013). Interestingly, the HIST1H2BJ RNA departs from the consensus $3^{\prime}$ end processing sequence and has a $C$ at position -1 relative to the stem loop sequence. Consistent with its consensus stem loop composition, the highly expressed $\mathrm{H} 2 \mathrm{~B}$ gene, $H I S T 1 H 2 B M$, was not found to be significantly polyadenylated in our studies in HCT116 cells or in DRS data for MCF7 or MCF10A cells. Thus, it appears likely that a canonical stem loop sequence promotes efficient histone mRNA $3^{\prime}$ end processing in vivo while single nucleotide changes in the loop or $5^{\prime}$ sequences may be sufficient to allow for alternative mRNA $3^{\prime}$ end processing via polyadenylation. 
In addition to expanding upon our previous observations that poly $\mathrm{A}^{+}$transcripts are produced from $\mathrm{H} 2 \mathrm{~B}$ genes, we have demonstrated that not all the $\mathrm{H} 2 \mathrm{~B}$ genes give rise to poly $^{+}$mRNAs. While the expression levels of the individual $\mathrm{H} 2 \mathrm{~B}$ genes frequently correlate with the amount of polyA $^{+}$transcripts produced, additional factors, including the composition of the $3^{\prime}$ stem loop sequence appear to influence the mode and efficiency of $3^{\prime}$ end processing. Given our recent findings that NPAT not only supports transcriptional regulation of histone genes, but also promotes proper $3^{\prime}$ end processing (Pirngruber and Johnsen, 2010), it is likely that sequences within the proximal promoter regions of the histone genes may also promote $3^{\prime}$ end processing.

Whether or not polyA ${ }^{+}$histone mRNAs play a physiological role remains unclear. However, since polyA ${ }^{+}$transcripts have longer half-lives compared to their $S$ phase counterparts (Kirsh et al., 1989), their expression may be necessary to compensate for decreased histone synthesis, for example in non-proliferating, terminally differentiated cells. Indeed, our results demonstrate that the levels of polyadenylated histone mRNAs significantly increase during cellular differentiation. Since in mRNA levels may not necessarily result in the production of a functional protein, we also performed polyribosome purification in cell cycle arrested cells and demonstrated for the first time that polyA ${ }^{+}$ transcripts are indeed polyribosomal and therefore likely give rise to functional histone proteins. However, whether the translated histone proteins produced from these transcripts are indeed incorporated into chromatin remains to be elucidated.

Since p53 accumulation following Nutlin-3a treatment mainly results in a prominent cell cycle arrest, we hypothesized that polyA $\mathrm{A}^{+}$transcript production is generally activated upon cell cycle arrest, when replication-dependent histone synthesis in not possible (Pirngruber and Johnsen, 2010). Moreover, we further hypothesized that conditions such as double-strand DNA break repair, which require massive changes in chromatin structure and histone exchange (Kari et al., 2011), may be particularly dependent upon polyA ${ }^{+}$histone transcripts for the generation of new histone proteins. In support of this hypothesis we observed an up-regulation of polyA ${ }^{+}$transcripts following $\gamma$-radiation. Based on these findings, we propose that induction of polyA $^{+}$histone transcripts may be a general mechanism to overcome a deficit in replication-dependent histone transcripts cause by cell cycle alterations. These transcripts may be essential for maintaining proper DNA packing 
and chromatin in the absence of replication where there is no expression of replicationdependent histone genes. Further studies are required to investigate the role of poly ${ }^{+}$ histone transcripts in cells or tissues like neurons or cardiomyocytes, which are terminally differentiated and no longer divide. Such studies will require the further elucidation of which genes encoding the other core histones are expressed and which of these are polyadenylated. In conclusion, our data demonstrate that production of poly ${ }^{+}$histone transcripts is subject to specific regulation and becomes induced during differentiation, DNA damage or cell cycle arrest most likely in order to maintain histone protein levels. 


\section{Materials and Methods}

\section{Cell culture and Nutlin-3a treatment}

HCT116 cells (human colon cancer cells) were grown in McCoy's medium containing $10 \%$ bovine growth serum (BGS; HyClone, USA) and 1x penicillin-streptomycin (Sigma, St. Louis, USA). Cells were either treated with vehicle (DMSO) or $8 \mu \mathrm{M}$ of Nutlin-3a (Sigma) for 24 hours and RNA was isolated. H1299 (human non-small cell lung carcinoma cell line), U2OS (human osteosarcoma cell line) and A549 (human alveolar adenocarcinoma cell line) cells were obtained from ATCC and grown in DMEM with high glucose medium containing $10 \%$ BGS, sodium pyruvate and 1x penicillinstreptomycin. Tert-immortalized human mesenchymal stem cells (hMSCs) (Simonsen et al., 2002) were kindly provided by M. Kassem, Odense University Hospital, Denmark. Cells were cultured in low glucose Minimum Essential Media (MEM) (Life Technologies, Carlsbad, USA) without glutamine and phenol red, supplemented with 10\% BGS and $1 \mathrm{x}$ antibiotic-antimycotic (Life Technologies). hFOB 1.19 cells were provided by Tom Spelsberg (Mayo Clinic, Rochester, Minnesota) and cultured at the permissive temperature $(34 \mathrm{C})$ in high glucose, phenol red free DMEM/F12 (Invitrogen) supplemented with 10\% BGS (Hyclone) and 1X penicillin-streptomycin (Invitrogen). Osteoblast differentiation was induced by shifting to the restrictive temperature $(39 \mathrm{C})$ and growing for 7 days. Adipocyte differentiation of hMSCs was induced as previously described (Karpiuk et al., 2012) by culturing cells in the presence of 15\% BGS, $10 \mathrm{nM}$ dexamethasone (Sigma), $0.45 \mathrm{mM}$ isobutylmethyl-xanthine (Sigma), $2 \mu \mathrm{M}$ insulin (Sigma), $10 \mu \mathrm{M}$ Troglitazone (Sigma) and 1x antibioticantimycotic solution. For osteoblast differentiation of hMSCs medium contained 10\% BGS, $10 \mathrm{nM}$ dexamethasone, $10 \mathrm{mM} \beta$-glycerol phosphate (BGP) (Sigma), $0.2 \mathrm{mM}$ ascorbic acid (Sigma), $10 \mathrm{nM}$ calcitriol (Cayman Chemicals, Ann Arbor, USA) and 1x antibiotic-antimycotic solution.

\section{Isolation of total RNA and cDNA preparation}

Total RNA was isolated from cells using TRIzol (Invitrogen) reagent according to the manufacturer's instructions. Polyadenylated mRNA was isolated from $100 \mu \mathrm{g}$ of total RNA using the PolyATtract ${ }^{\circledR}$ mRNA Isolation System III (Cat. No. Z5300, Promega, Wisconsin) according to the manufacturer's instructions. Total or polyA ${ }^{+}$RNA was reverse transcribed using either random nonamers or polyT primers as indicated in the figure legends. CDNA samples were analyzed by SYBR Green based quantitative real time PCR (qRT-PCR) as described (Pirngruber and Johnsen, 2010). The expression of individual H2B genes was measured in various cell lines using a linear dilution curve of genomic DNA (gDNA) with known concentrations from a normal, diploid cell line (hMSCs). Finally, relative $\mathrm{H} 2 \mathrm{~B}$ expression from each gene in different cell lines was normalized to the genomic DNA dilution curve (assuming that each gene is equally represented in a diploid cell) and indicated as 
"Rel. gDNA units". The sequences of primers utilized in this study are listed in Supplementary Table S1.

\section{Cytoplasmic RNA preparation}

For the examination of nuclear and cytoplasmic RNA, HCT116 cells were treated with DMSO or Nutlin-3a for 24 hours and lysed in buffer containing $50 \mathrm{mM}$ Tris $\mathrm{HCl}(\mathrm{pH} 8.0), 140 \mathrm{mM} \mathrm{NaCl}, 1.5$ $\mathrm{mM} \mathrm{MgCl} 2,0.5 \% \mathrm{v} / \mathrm{v}$ Igepal and $1000 \mathrm{U} / \mathrm{ml}$ RNase Inhibitor. Cytoplasmic and nuclear fractions were separated by centrifugation at 900 RPM for 10 minutes. RNA was isolated from the cytoplasmic fraction using standard TRIzol extraction method. Unprocessed rRNA and spliced RPLPO were used as positive controls for the nuclear and cytoplasmic fractions, respectively (Supplemental Figure S2).

\section{Polyribosome purification}

Polyribosome purification was carried out essentially as reported with slight modifications (Mašek et al., 2011). Briefly, HCT116 cells were treated with DMSO or Nutlin-3a for 24 hours and cells were treated with cycloheximide at $37^{\circ} \mathrm{C}$ for $30 \mathrm{~min}$ brought to the final concentration of 100 $\mu \mathrm{g} / \mathrm{ml}$. Cell lysates were prepared in lysis buffer containing $20 \mathrm{mM}$ HEPES (pH 7.5), $125 \mathrm{mM} \mathrm{KCl}, 5$ $\mathrm{mM} \mathrm{MgCl}, 2 \mathrm{mM} \mathrm{DTT}, 0.5 \% \mathrm{NP}-40,100 \mu \mathrm{g} / \mathrm{ml}$ of cycloheximide and $100 \mathrm{U} / \mathrm{ml}$ of RNase inhibitor along with protease inhibitors. Cleared lysates were loaded on to the sucrose gradient 8-50 \% in lysis buffer and centrifuged at 34,000 RPM for 130 minutes. Fractions were collected from the gradients and RNA was extracted from polyribosome fractions. 


\section{I.1 Supplementary Figures}

A

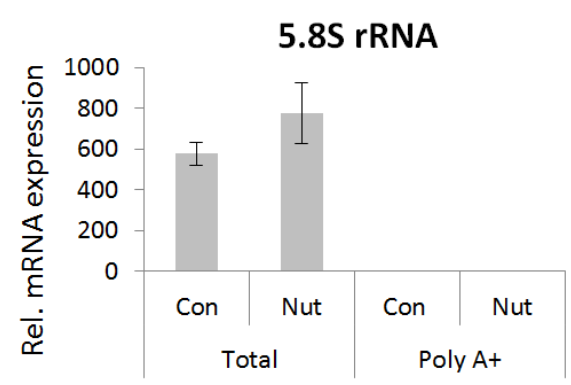

18S rRNA

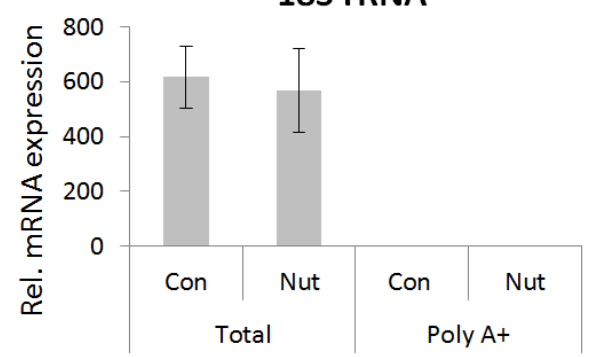

B

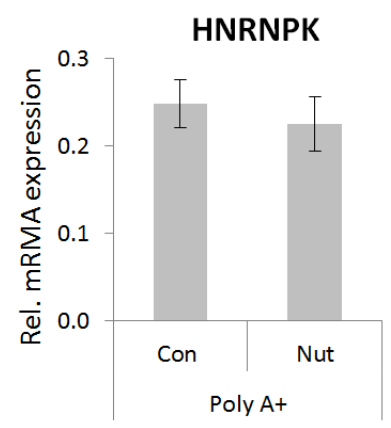

Supp Fig I.S1: (A) Quality of polyA ${ }^{+}$mRNA purified using PolyA Ttract ${ }^{\circledR}$ mRNA Isolation System III.

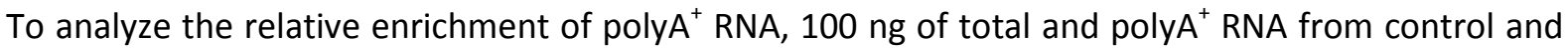
Nutlin-3a treated cells was reverse transcribed using random nonamers and analyzed for $5.8 \mathrm{~S}$ and $18 \mathrm{~S}$ rRNA transcripts by qRT-PCR. (B) qRT-PCR analysis for HNRNPK mRNA expression in polyA ${ }^{+}$ purified mRNA from control and Nutlin-3a treated cells.

A

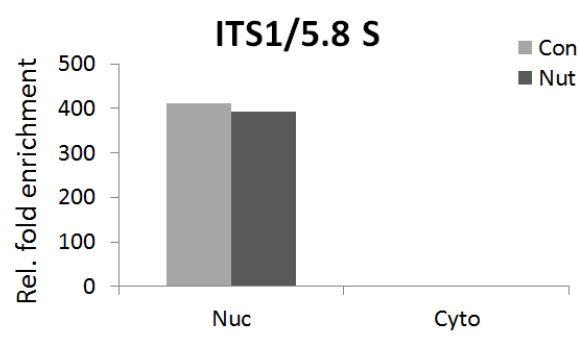

B

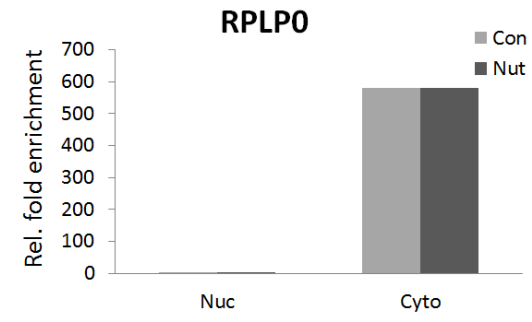

Supp Fig I.S2: Purity of cytoplasmic and nuclear RNA. To check the purity of cytoplasmic and nuclear fractions RNA was analyzed by qRT-PCR for (A) un-spliced 5.8 S rRNA (specific for nuclear), (B) RFLPO (cytoplasmic) from control and Nutlin-3a treated cells. 


\section{Supp Table I.S1: Primers used in this study 5' to 3' orientation.}

Name

ALPL F

ALPLR

BGLAP F

BGLAP R

HIST1H2BA $145 \mathrm{~F}$

HIST1H2BA 246 R

HIST1H2BB $151 \mathrm{~F}$

HIST1H2BB $352 \mathrm{R}$

HIST1H2BC $47 \mathrm{~F}$

HIST1H2BC $183 \mathrm{R}$

HIST1H2BD $47 \mathrm{~F}$

HIST1H2BD $115 \mathrm{R}$

HIST1H2BE $55 \mathrm{~F}$

HIST1H2BE $174 \mathrm{R}$

HIST1H2BF $157 \mathrm{~F}$

HIST1H2BF 340 R

HIST1H2BG $84 \mathrm{~F}$

HIST1H2BG $252 \mathrm{R}$

HIST1H2BH $87 \mathrm{~F}$

HIST1H2BH $323 \mathrm{R}$

HIST1H2BI $278 \mathrm{~F}$

HIST1H2BI $421 \mathrm{R}$

HIST1H2BJ $55 \mathrm{~F}$

HIST1H2BJ $156 \mathrm{R}$

HIST1H2BK 8

HIST1H2BK $152 \mathrm{R}$

HIST1H2BL $69 \mathrm{~F}$

HIST1H2BL 196

HIST1H2BM $291 \mathrm{~F}$ HIST1H2BM $320 \mathrm{R}$ HIST1H2BN 61F

HIST1H2BN 162R

HIST1H2BO $23 \mathrm{~F}$

HIST1H2BO $146 \mathrm{R}$

HIST2H2BE $767 \mathrm{~F}$

HIST2H2BE $958 \mathrm{R}$

HIST2H2BF $241 \mathrm{~F}$

HIST2H2BF $397 \mathrm{R}$

HIST3H2BB $232 \mathrm{~F}$

HIST3H2BB $423 \mathrm{R}$

HIST1H2BD PolyA F

HIST1H2BD PolyA R

HIST1H2AC Total F

HIST1H2AC Total R

HIST1H2AC PolyA

HIST1H2AC PolyA R

HIST1H2BD Spl 402 F

HIST1H2BD Spl 614 R

HIST1H2AC Spl 505F

HIST1H2AC Spl 632R

HNRNPK F

HNRNPK R

PPARG F

PPARG R

RPLPO F

RPLPO R

rRNA ITS1/5.8S F

rRNA ITS1/5.8S R

rRNA 5.8S F

rRNA $5.8 S \mathrm{R}$

h18S rRNA F

h18S rRNA R
Sequence (5'-3')

TGGGCCAAGGACGCTGGGAA

AAGGCCTCAGGGGGCATCTCG

GCCCTCACACTCCTCGCCCT

CGGGTAGGGGACTGGGGCTC

CAGGTCCATCCGGACACTGGCA

CAAACGTGATGCCTCGCTCGCT

CCCGACACCGGCATCTCATCCA

CCTTAGTGCCCTCGGACACAGCA

AGAAGGCAGTGACCAAAGCGCAG

GCCCATGGCCTTGGAAGAGATGC

ACGATGCCTGAACCTACCAA

AGCCTTAGTCACCGCCTTCT

GTGACCAAGGCGCAGAAGAAGGAC

TTTAGAGGAGATGCCGGTGTCGGG

ACCGGCATCTCATCCAAGGCCA

TGACACGGCGTGCTTAGCCAG

AGAAGCGCAAGCGCAGTCGT

TAGTGGGCCAGACGGGAAGCC

GCGTAAACGCAGCCGCAAGG

GCCAGTTCCCCAGGCAGCAG

GGGAGATCCAAACGGCTGTGCG

GAGCCTTTGGGTCGTTAGCGCTTT

GCCAGCGAAGTCTGCTCCCG

CTCTCCTTGCGGCTGCGCTT

TGCTGCTCGTCTCAGGCTCGT

CTCTCCTTGCGGCTGCGCTT

CCAAGAAGGCGGTGACCAAGGC

AGAAGAGATGCCGGTGTCGGGG

GGCCGTGCGCCTACTGCTAC

GGTGTGGGTCACGGCGGAAC

CAAAGTCCGCTCCTGCCCCG

TGACCGAACGTTCCGCGGTG

TTCACTCTCCTCCGCCATGCCC

CTCTTTGCGGCTGCGCTTGC

CCTGGTGGCTCCTTGGGTCTGT

TATCCACAGGAGGCCCCATCGC

CCTCCACCCCACCACCCCTC

ATGGACTCGGGAACCGCCGA

TCTTCGAGCGCATCGCCAGC

CAGGACGCCGAGGAACGCC

CCAACTCATCCTGGTTTGCT

TCCCCTCGGTAACCTTCTTT

GACGAGGAGCTCAACAAACTG

ACCTGTCAAATCACTTGCCC

CCTGTCCACTGTTGGTAGGC

TTCACTTACCACCATTCCAGC

CCGTCACCAAGTACACCAGTT

TCCCCTCGGTAACCTTCTTT

CCCCTACCGTTTCAAAGGA

ATTGGTAAGTTTGGCAGGCA

ATCCGCCCCTGAACGCCCAT

ACATACCGCTCGGGGCCACT

ACCTCCGGGCCCTGGCAAAA

TGCTCTGCTCCTGCAGGGGG

GATTGGCTACCCAACTGTTG

CAGGGGCAGCAGCCACAAA

GGCCTGAGGCAACCCCCTCT

GACGCACGAGCCGAGTGATCC

GCGGTGGATCACTCGGCTCG

CGTAGCCCCGGGAGGAACCC

AACTGAGGCCATGATTAAGA

GGAACTACGACGGTATCTGA
Reference (if present)

(Karpiuk, 2012)

(Karpiuk, 2012)

(Karpiuk, 2012)

(Karpiuk, 2012)

This study

This study

This study

This study

This study

This study

This study

This study

This study

This study

This study

This study

This study

This study

This study

This study

This study

This study

This study

This study

This study

This study

This study

This study

This study

This study

This study

This study

This study

This study

This study

This study

This study

This study

This study

This study

(Pirngruber et al., 2009)

(Pirngruber et al., 2009)

(Pirngruber et al., 2009)

(Pirngruber et al., 2009)

(Pirngruber et al., 2009)

(Pirngruber et al., 2009)

This study

This study

This study

(Karpiuk, 2012)

(Karpiuk, 2012)

(Karpiuk, 2012)

(Karpiuk, 2012

(Fritah et al., 2005)

(Fritah et al., 2005)

This study

This study

This study

This study

This study

This study 


\subsection{Publication II}

\section{The H2B ubiquitin ligase RNF40 cooperates with SUPT16H to induce dynamic changes in chromatin structure during DNA double-strand break repair}

\section{Citation}

Vijayalakshmi Kari, Andrei Shchebet, Heinz Neumann and Steven A. Johnsen

The H2B ubiquitin ligase RNF40 cooperates with SUPT16H to induce dynamic changes in chromatin structure during DNA double-strand break repair

Cell Cycle. 2011, 10(20):3495-504: http://dx.doi.org/10.4161/cc.10.20.17769

\section{Own contribution}

Preparation and accomplishment of the biological experiments presented in Fig II.1, Fig II.2, Fig II.3, Fig II.4, Supp Fig II.S1 and Supp Fig. II.S2. i.e cell culture, NCS treatment, chromatin fractionation, western blot, immunofluorescence, FACS analysis, comet assay. Complete figure layout and contribution to the writing manuscript. 


\section{Abstract}

Many anti-cancer therapies function largely by inducing DNA double-strand breaks (DSBs) or altering the ability of cancer cells to repair them. Proper and timely DNA repair requires dynamic changes in chromatin assembly and disassembly characterized by histone H3 lysine 56 acetylation (H3K56ac) and phosphorylation of the variant histone $\mathrm{H} 2 \mathrm{AX}(\mathrm{\gamma H} 2 \mathrm{AX})$. Similarly, histone H2B monoubiquitination (H2Bub1) functions in DNA repair, but its role in controlling dynamic changes in chromatin structure following DSBs and the histone chaperone complexes involved remain unknown. Therefore, we investigated the role of the H2B ubiquitin ligase RNF40 in the DSB response. We show that RNF40 depletion results in sustained H2AX phosphorylation and a decrease in rapid cell cycle checkpoint activation. Furthermore, RNF40 knockdown resulted in decreased H3K56ac and decreased recruitment of the Facilitates Chromatin Transcription (FACT) complex to chromatin following DSB. Knockdown of the FACT component Suppressor of Ty Homologue-16 (SUPT16H) phenocopied the effects of RNF40 knockdown on both $\mathrm{YH} 2 \mathrm{AX}$ and H3K56ac following DSB induction. Consistently, both RNF40 and SUPT16H were required for proper DNA end resection and timely DNA repair suggesting that $\mathrm{H} 2 \mathrm{Bub1}$ and FACT cooperate to increase chromatin dynamics which facilitates proper checkpoint activation and timely DNA repair. These results provide important mechanistic insights into the tumor suppressor function of H2Bub1 and provide a rational basis for pursuing H2Bub1-based therapies in conjunction with traditional chemo- and radiotherapy.

\section{Introduction}

The induction of DNA double-strand breaks (DSBs) from exogenous and endogenous sources poses a significant threat to genomic integrity. The improper recognition and repair of DSBs increases the probability of tumorigenesis but may also be exploited for the treatment of cancer in combination with radio- or chemotherapy. Like other DNA-associated processes such as transcription and DNA replication, the winding of the DNA around the

histone octamer and packaging into higher order chromatin structures represents a significant barrier for DNA repair (Rossetto et al., 2010). In order to perform these processes rapid and highly dynamic alterations in chromatin must occur including changes in the posttranslational modification of core histones and rearrangement of chromatin structure 
by histone chaperones and chromatin remodeling complexes. It has been proposed that DSB repair in the context of chromatin occurs in a step-wise fashion including: (1) recognition of the DSB, (2) rearrangement of chromatin to allow access of repair enzymes to the damaged site, (3) repair of the DSB, and (4) re-establishment of chromatin structure (Ransom et al., 2010). The role of chromatin dynamics, post-translational histone modifications and their mechanisms of action during the DNA damage response (DDR) and DNA repair is an intensively studied and highly interesting area of cancer research which remains poorly understood.

A number of histone modifications have been identified which may play roles during DDR and DNA repair in mammals. For example, the variant histone $\mathrm{H} 2 \mathrm{AX}$ is modified at multiple residues following ionizing radiation (Xie et al., 2010). Importantly, Ser139 phosphorylation $(\gamma \mathrm{H} 2 \mathrm{AX})$ by the phosphatidyl-inositol-3-kinase family members DNA-PK, ATM and ATR in humans is perhaps one of the most widely used and important biomarkers of DNA damage (Redon et al., 2011). Phosphorylation of H2AX accompanies dynamic alterations in chromatin structure (Dellaire et al., 2009; Massip et al., 2010), at least in part by recruiting BRCT domain-containing proteins such as the Mediator of DNA Checkpoint-1 (MDC1) which are involved in DNA repair (Ciccia and Elledge, 2010) and induce chromatin relaxation (Nakamura et al., 2010). The recruitment of MDC1 serves to recruit additional DNA repair proteins and propagate $\mathrm{\gamma H} 2 \mathrm{AX}$ across a larger region surrounding the DSB.

The phosphorylation of $\mathrm{H} 2 \mathrm{AX}$ occurs simultaneously with the acetylation of histone H3 lysine 56 (H3K56ac) in response to genotoxic stress (Das et al., 2009; Vempati et al., 2010; Yuan et al., 2009). In vitro studies using a synthetic biology approach are beginning to unravel the molecular details of how this modification functions during chromatin assembly and disassembly (Neumann et al., 2009). In vivo, H3K56 is acetylated by Rtt109 in yeast (Burgess and Zhang, 2010; Chen et al., 2008; Li et al., 2008) and p300 (Das et al., 2009; Vempati et al., 2010) and/or CBP (Das et al., 2009) in mammalian cells and deacetylated by the Sirtuin family members Hst3 and Hst4 in yeast (Celic et al., 2006; Maas et al., 2006) and Sirt1 (Das et al., 2009; Yuan et al., 2009), Sirt2 (Das et al., 2009; Vempati et al., 2010) and/or Sirt3 (Vempati et al., 2010) in mammalian cells. Interestingly, another mammalian Sirtuin family member Sirt6 is also involved in DNA repair by interacting directly with DNA-PK (McCord et al., 2009). H3K56ac is required for chromatin reassembly both during DNA 
replication and after DNA damage in yeast (Burgess and Zhang, 2010; Chen et al., 2008). Similarly, the presence of H3K56ac in chromatin in humans occurs only after nucleosome reassembly and reflects recent changes in chromatin dynamics following DNA damage (Das et al., 2009; Ransom et al., 2010).

Another histone modification which was recently shown to be essential for proper DNA repair is the monoubiquitination of histone $\mathrm{H} 2 \mathrm{~B}$ at lysine 120 ( $\mathrm{H} 2 \mathrm{Bub} 1)$ by the ubiquitin ligase RNF20 (Chernikova et al., 2010; Moyal et al., 2011; Nakamura et al., 2011). A loss of H2Bub1 resulted in a prolonged DNA damage response (e.g., H2AX phosphorylation), decreased formation of RAD51 foci and decreased DNA repair without inhibiting the rapid induction of H2AX phosphorylation (Moyal et al., 2011; Nakamura et al., 2011). In mammals $\mathrm{H} 2 \mathrm{~B}$ is monoubiquitinated by the obligate heterodimeric ubiquitin ligase complex containing both RNF20 and RNF40 (Kim et al., 2009; Pavri et al., 2006; Zhu et al., 2005) and is associated with the transcribed regions of active genes (Minsky et al., 2008). Our previous work revealed a tumor suppressor role for RNF20 and demonstrated an essential function of cyclin-dependent kinase-9 (CDK9) in maintaining H2Bub1 (Pirngruber et al., 2009a, 2009b; Shema et al., 2008). The tumor suppressor function of H2Bub1 was also supported by our recent demonstration that a decrease in H2Bub1 levels strongly correlates with breast cancer progression (Prenzel et al., 2011). However, a mechanistic role for H2Bub1 during tumorigenesis and DNA repair has remained unclear.

H2Bub1 likely functions during both transcription and DNA repair by altering the dynamics of histone exchange. One potential mediator of these effects is the histone chaperone complex FACT (Facilitates Chromatin Transcription) which contains the Structure Specific Recognition Protein-1 (SSRP1) and Suppressor of Ty Homologue-16 (SUPT16H). Importantly, H2Bub1 and FACT were shown to cooperate during transcriptional elongation in vitro (Pavri et al., 2006) and a decrease of H2Bub1 following proteasome inhibitor treatment led to a loss of chromatin-bound SSRP1 and decreased histone exchange (Prenzel et al., 2011). Furthermore, the induction of DSB by $\gamma$-irradiation increases the nucleoplasmic pool of histone $\mathrm{H} 3$, and this effect is lost following RNF2O knockdown, suggesting that H2Bub1 may be essential for DSB-induced nucleosome dynamics (Nakamura et al., 2011). Like H2Bub1, FACT may also play a role in DNA repair. For example, SSRP1 recognizes cisplatin DNA adducts (Dejmek et al., 2009; Yarnell et al., 2001) and is recruited to sites of 
DNA microirradiation (Sand-Dejmek et al., 2011). Furthermore, FACT catalyzes the exchange of $\mathrm{yH} 2 \mathrm{AX}$-containing H2AX-H2B dimers on nucleosomes in vitro (Heo et al., 2008). However, the roles and interaction of $\mathrm{H} 2 \mathrm{Bub1}$ and FACT in DSB repair, and their effects on damageinduced nucleosome dynamics in vivo remain unexplored. In this manuscript we investigated the roles and interactions of RNF40 and SUPT16H during the cellular DNA DSB response and provide the first in vivo evidence that FACT plays an essential H2Bub1dependent role in causing dynamic changes in chromatin structure which are required for cell cycle checkpoint activation and DNA repair.

\section{Results}

\section{RNF40 knockdown leads to a prolonged DNA damage response}

Recent work from a number of laboratories uncovered a critical role for H2Bub1 and the H2B ubiquitin ligases RNF20 and RNF40 in DNA DSB repair (Chernikova et al., 2010; Moyal et al., 2011; Nakamura et al., 2011). Given the importance of H2AX phosphorylation in the DNA damage response, we performed siRNA-mediated knockdown of the $\mathrm{H} 2 \mathrm{~B}$ ubiquitin ligase RNF40 and analyzed $\mathrm{\gamma H} 2 \mathrm{AX}$ levels by Western blot in the chromatin-bound fractions of cells treated with the radiomimetic compound neocarzinostatin (NCS) for various time points. As shown in Fig II.1A, we observed no effect of RNF40 knockdown on the induction of $\mathrm{\gamma H} 2 \mathrm{AX}$ levels at 30 minutes after DSB induction suggesting an intact DNA damage response.

A

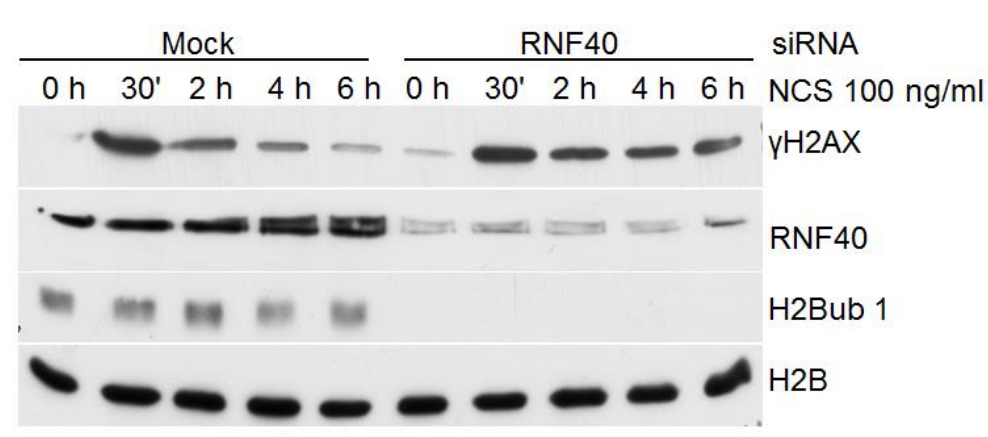


B

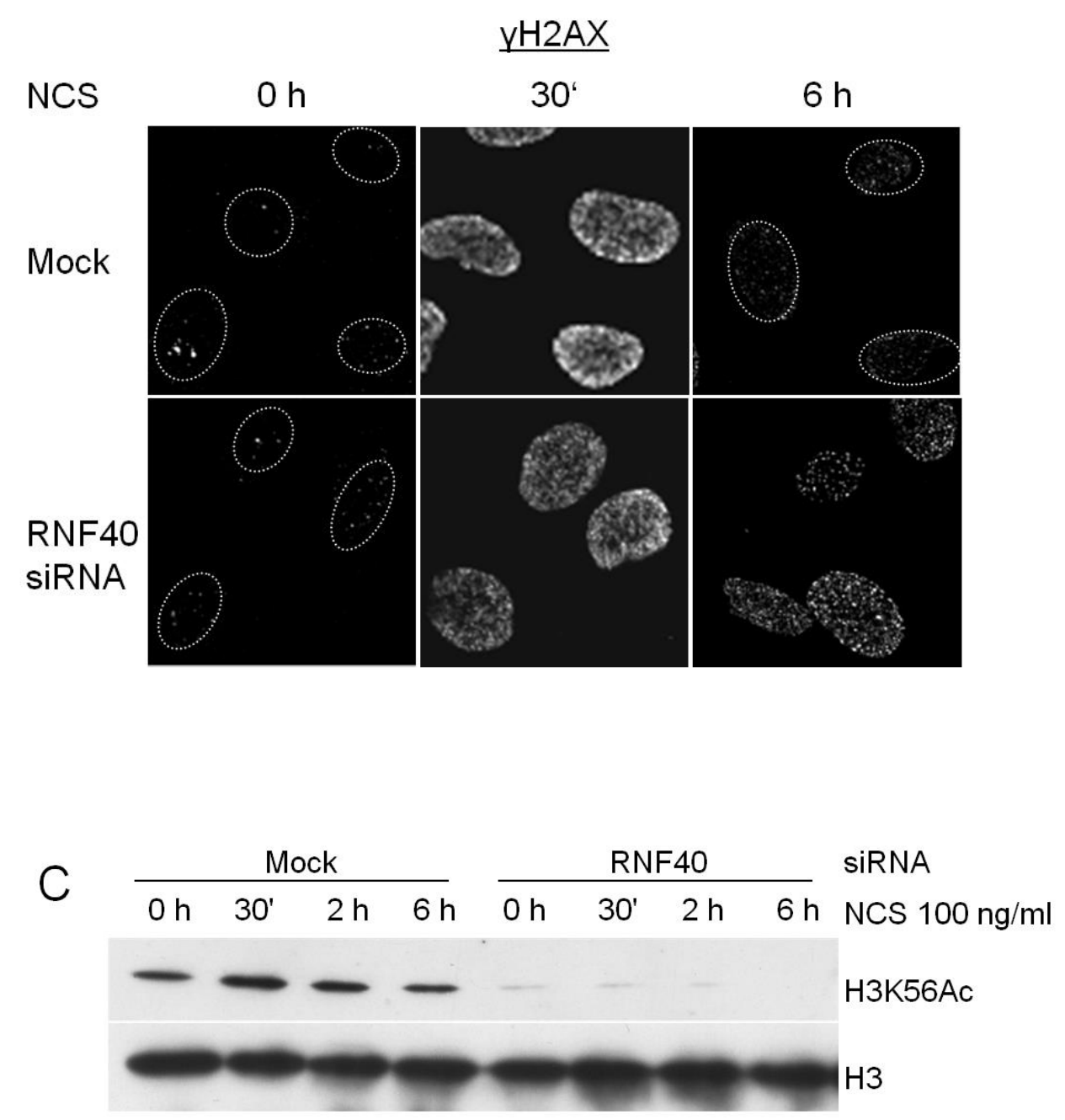

D

H3K56ac

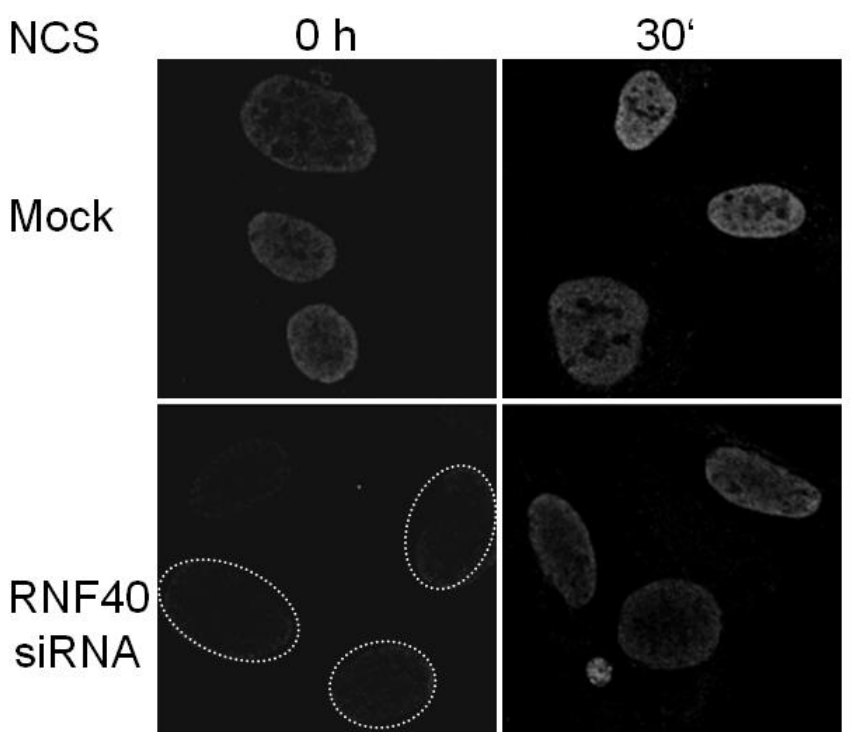


Fig II.1: RNF40 knockdown leads to the prolonged pH2AX accumulation and loss of H3K56ac increase after NCS treatment. (A) U2OS cells were transfected with RNF40 siRNA or mock for 48 hours and treated with $100 \mathrm{ng} / \mathrm{ml} \mathrm{NCS}$ for the indicated times. Chromatin fractions were prepared and analyzed by Western blot for $\mathrm{YH} 2 \mathrm{AX}, \mathrm{H} 2 \mathrm{Bub} 1$ and $\mathrm{H} 2 \mathrm{~B}$. (B) U2OS cells were transfected and immunohistochemically stained against $\mathrm{\gamma H} 2 \mathrm{AX}$ at the indicated time points following NCS treatment. (C) RNF40 is required for rapid histone exchange after DNA damage. U2OS cells were transfected with RNF40 siRNA or mock for 48 hours and treated with NCS as in (a) for the indicated time points and whole cell lysates were immunoblotted for H3K56ac and H3. (D) Similar to (B) cells were immunostained with an H3K56ac antibody at the indicated time points following NCS treatment.

However, while $\mathrm{pH} 2 \mathrm{AX}$ levels significantly declined at 4 and 6 hours following NCS treatment of control transfected cells, they remained elevated in RNF40 depleted cells. In order to test whether the increased $\mathrm{\gamma H} 2 \mathrm{AX}$ levels were contributed by a subpopulation of cells or the whole population of cells displayed a uniform increase we performed confocal immunofluorescence analyses. As shown in Fig II.1B, the increase in $\mathrm{yH} 2 \mathrm{AX}$ staining 6 hours after NCS treatment was uniformly higher in RNF40-depleted cells. Thus we conclude that RNF40 knockdown results in a prolonged DNA damage response following DSB.

\section{The induction of H3K56ac is impaired following RNF40 knockdown}

Recent studies demonstrated that histone H3 is rapidly acetylated at lysine 56 following DNA damage with similar kinetics and overlapping localization with $\mathrm{\gamma H} 2 \mathrm{AX}$ (Das et al., 2009; Vempati et al., 2010). Furthermore, H3K56ac is coupled to transcriptionassociated chromatin reassembly (Vempati et al., 2010). Therefore, we also tested whether $\mathrm{H} 3 \mathrm{~K} 56 \mathrm{ac}$ is affected by a loss of H2Bub1. Surprisingly, in contrast to $\mathrm{YH} 2 \mathrm{AX}$, whose rapid induction was not affected by RNF40 knockdown, we observed a dramatic decrease in both the basal and DSB-induced levels of H3K56ac at all-time points (Fig II.1C). These results were corroborated by immunofluorescence analyses (Fig II.1D). Thus, we demonstrate the first example of divergent effects of DNA-damage on the induction of $\mathrm{\gamma H} 2 \mathrm{AX}$ and H3K56ac and a dependence of both DSB-induced and transcription-coupled H3K56ac on RNF40 activity.

\section{RNF40 knockdown decreases cell cycle checkpoint activation}

Following the induction of DSB, cells normally activate cell cycle checkpoints which prevent DNA replication and/or entry into mitosis before repair has been completed. Since defects in cell cycle checkpoints can lead to genomic instability and ultimately to tumorigenesis, we analyzed the effects of RNF40 depletion on the activation of cell cycle checkpoints after the induction of DSB. One of the essential mediators of cell cycle 
checkpoint activation is the CHK2 kinase which is phosphorylated and activated by ATM following DSB (Antoni et al., 2007). Therefore, we investigated whether RNF40 depletion influenced CHK2 activation following NCS treatment. Indeed, knockdown of RNF40 decreased CHK2 phosphorylation already 15 minutes after NCS treatment without significantly affecting H2AX phosphorylation (Fig II.2A). Moreover, CHK2 activation was potentiated by RNF40 overexpression and the effects of RNF40 knockdown could be rescued by RNF40 overexpression (Supp Fig II.1).

A

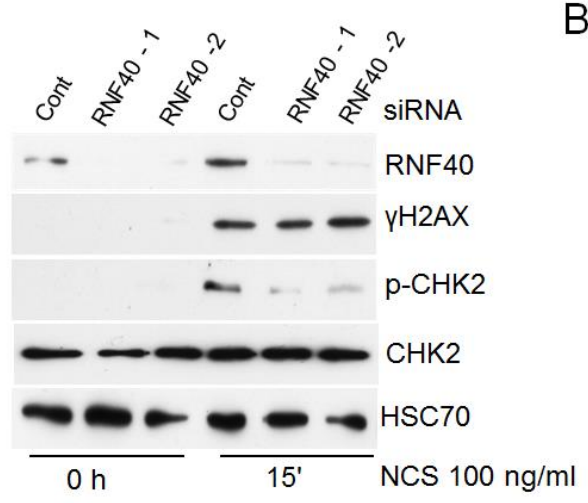

B

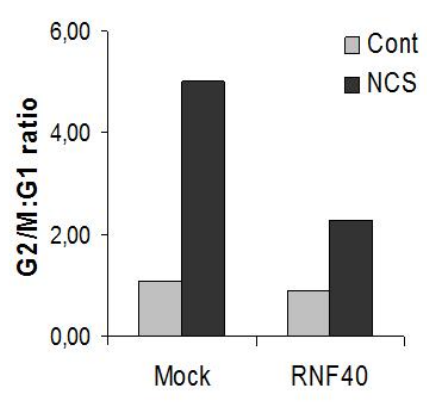

C

Mock

RNF40 SiRNA

Cont

NCS

Cont

NCS
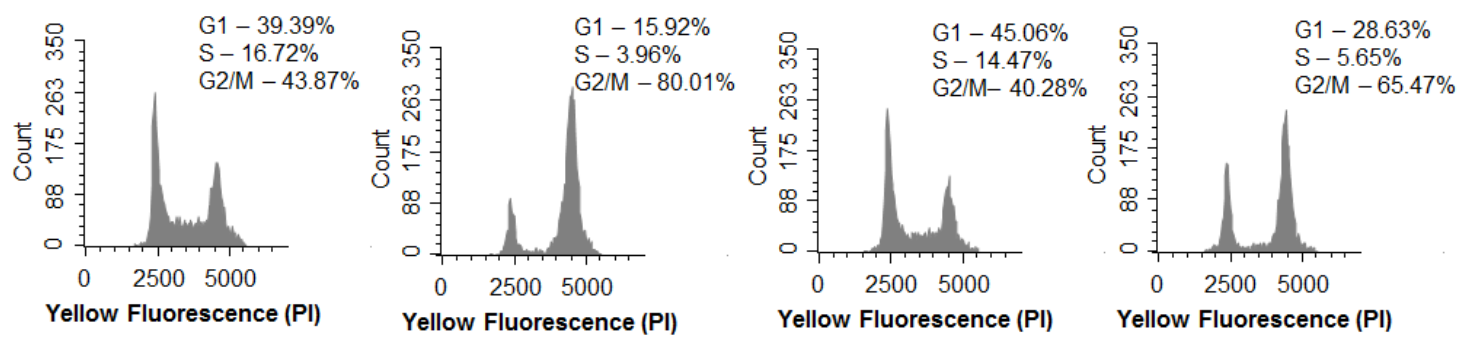

D

Mock

NCS
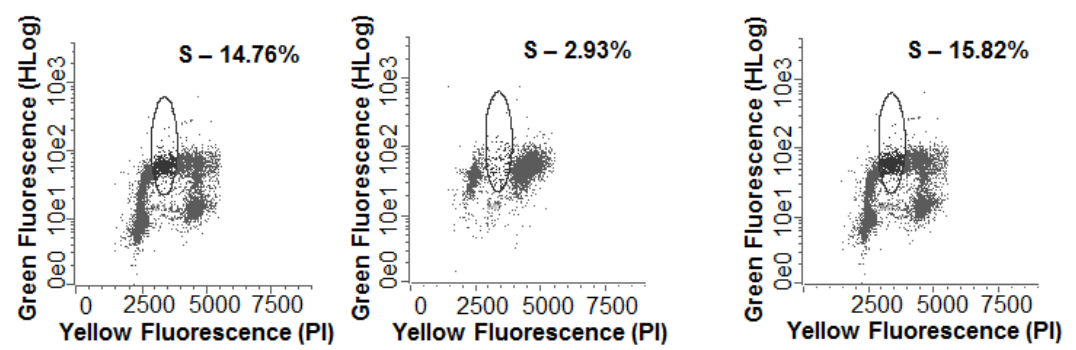

NCS

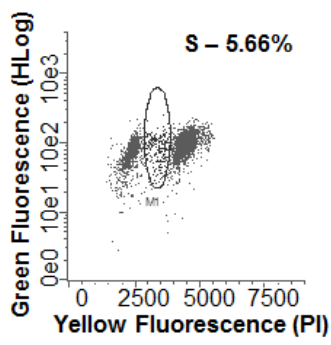


Fig II.2: RNF40 is critical for cell cycle checkpoint activation. (A) U2OS cells were transfected with two different RNF40 siRNAs or mock control for 48 hours and were treated with $100 \mathrm{ng} / \mathrm{ml} \mathrm{NCS}$ for $15 \mathrm{~min}$. Whole cell lysates were analysed by Western blot for RNF40, $\mathrm{YH} 2 \mathrm{AX}, \mathrm{p}-\mathrm{CHK} 2$ and HSC70. (B D) HCT116 cells were transfected with RNF40 siRNA or mock for 48 hours before treatment with 100 $\mathrm{ng} / \mathrm{ml} \mathrm{NCS}$ for an additional 12 hours as indicated followed by processing for propidium iodide and BrdU-based flow cytometry. (B-C) The ratio for the G2/M to G1 fraction is graphically represented based in the bar graph (B) and the propidium iodide profile of cells examined is shown in (C). (D) BrdU incorporation based cell profile examined in (B).

Based on the effects on CHK2 activation we investigated whether RNF40 knockdown also affected DSB-induced changes in the cell cycle profile. Indeed, while NCS induced a significant increase in the G2/M fraction in control cells, RNF40 knockdown decreased the G2/M to G1 ratio from 5.02 in control cells to only 2.29 in RNF40 depleted cells following NCS treatment (Fig II.2B and C). Furthermore, consistent with cell cycle checkpoint activation, NCS treatment led to a significant reduction in the $S$ phase fraction of cells (14.76\% to $2.93 \%)$ in control cells as assessed by BrdU-based flow cytometric analyses. Knockdown of RNF40 impaired the G1/S cell cycle checkpoint activation where RNF40depleted cells demonstrated a higher fraction of $S$ phase cells following NCS treatment (5.66\% compared to $2.93 \%$ in control transfected cells; Fig II.2D). RNF40 knockdown had little effect on the S phase fraction of normally cycling cells ( $14.76 \%$ compared to $15.82 \%)$. Thus we conclude that RNF40 expression is essential for the induction and maintenance of cell cycle checkpoint activation following DSB induction.

\section{RNF40 expression is essential for FACT recruitment to chromatin}

The presence of H3K56ac following DNA damage marks chromatin which has undergone dynamic disassembly and reassembly since it is dependent upon the H3-H4 histone chaperone activity of Anti-silencing Factor-1a (ASF1a) (Das C, Ransom M). Chromatin assembly in vivo occurs in an ordered chaperone-dependent process in which two H3-H4 dimers initially form a tetramer to which two H2A-H2B dimers are subsequently added to form the intact nucleosome octamer (Ransom et al., 2010). Similarly, during chromatin disassembly, the two H2A-H2B dimers must also be displaced before the H3-H4 dimers can be removed. Therefore, we hypothesized that H3K56ac may also require the activity of an $\mathrm{H} 2 \mathrm{~A}-\mathrm{H} 2 \mathrm{~B}$ chaperone. Consistent with a potential role in DNA repair, the FACT complex was shown to catalyze the exchange of $\mathrm{yH} 2 \mathrm{AX}-\mathrm{H} 2 \mathrm{~B}$ dimers in vitro (Heo et al., 2008) and also interacts with H3K56ac in yeast (Nair et al., 2011). Furthermore, H2Bub1 was 
shown to cooperate with FACT during transcriptional elongation (Pavri et al., 2006) whereby it was proposed that FACT catalyzes an exchange of histone $\mathrm{H} 2 \mathrm{~A}-\mathrm{H} 2 \mathrm{~B}$ dimers in an H2Bub1dependent manner (Laribee et al., 2007). Based on the effects of RNF40 knockdown on H3K56ac, we hypothesized that a loss of H2Bub1 may lead to impaired histone exchange following DNA damage through decreased FACT recruitment. However, whether H2Bub1 is required for FACT recruitment to chromatin in humans remains unknown. Therefore, we first analyzed the recruitment of the FACT components SUPT16H and SSRP1 to chromatin in the presence and absence of DSB. Consistent with its role in transcriptional elongation and its proposed role in the DDR, we observed an increased FACT binding to chromatin following NCS treatment (Fig II.3A). Importantly, the binding of both FACT components (SSRP1 and SUPT16H) after NCS treatment was decreased following RNF40 knockdown. Importantly, RNF40 knockdown did not affect the expression of FACT components or vice versa (Supp Fig II.2).

We next tested whether the FACT complex is essential for mediating the effects of H2Bub1 during the DDR. Therefore, we performed siRNA-mediated knockdown of SUPT16H and analyzed the effects on $\mathrm{YH} 2 \mathrm{AX}$ and H3K56ac. Similar to the results with RNF40 knockdown, we observed no effect of SUPT16 $\mathrm{H}$ knockdown on the induction of $\mathrm{YH} 2 \mathrm{AX}$ at 30 minutes, but rather a sustained induction up to 6 hours following NCS treatment (Fig II.3B). These results were confirmed by confocal immunofluorescence analyses in which a uniform increase in $\mathrm{YH} 2 \mathrm{AX}$ was observed 6 hours after NCS treatment in SUPT16H knockdown cells (Fig II.3C). Similar to the effects observed following RNF40 knockdown, both basal and DSB induced levels of H3K56ac were significantly reduced following NCS treatment of SUPT16Hdepleted cells (Fig II.3D and E). Thus these results suggest that, like RNF40, SUPT16H is required for termination of the DDR and increased chromatin dynamics following DSB induction. 
A

\begin{tabular}{|c|c|c|c|c|c|c|c|c|}
\hline \multicolumn{4}{|c|}{ Mock } & \multicolumn{4}{|c|}{ RNF40 } & siRNA \\
\hline $\mathrm{Oh}$ & $30^{\prime}$ & $2 \mathrm{~h}$ & $6 \mathrm{~h}$ & $\mathrm{Oh}$ & $30^{\prime}$ & $2 \mathrm{~h}$ & $6 \mathrm{~h}$ & NCS $100 \mathrm{ng} / \mathrm{m}$ \\
\hline - & - & - & - & $\cdots$ & $=$ & - & - & SUPT16H \\
\hline$\ldots$ & - & $=$ & - & $=$ & - & - & - & SSRP1 \\
\hline
\end{tabular}

B

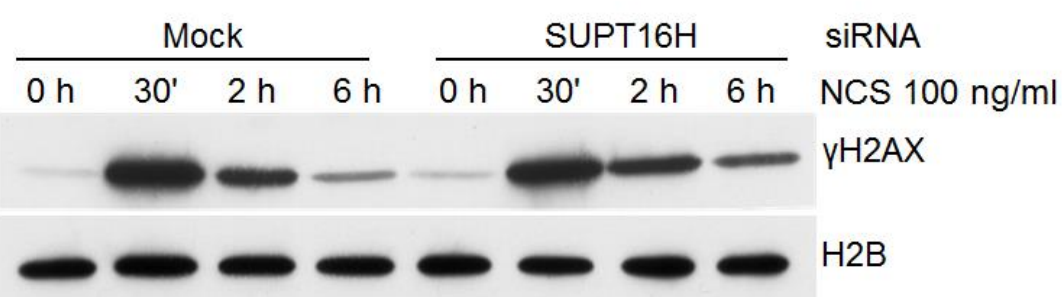

C

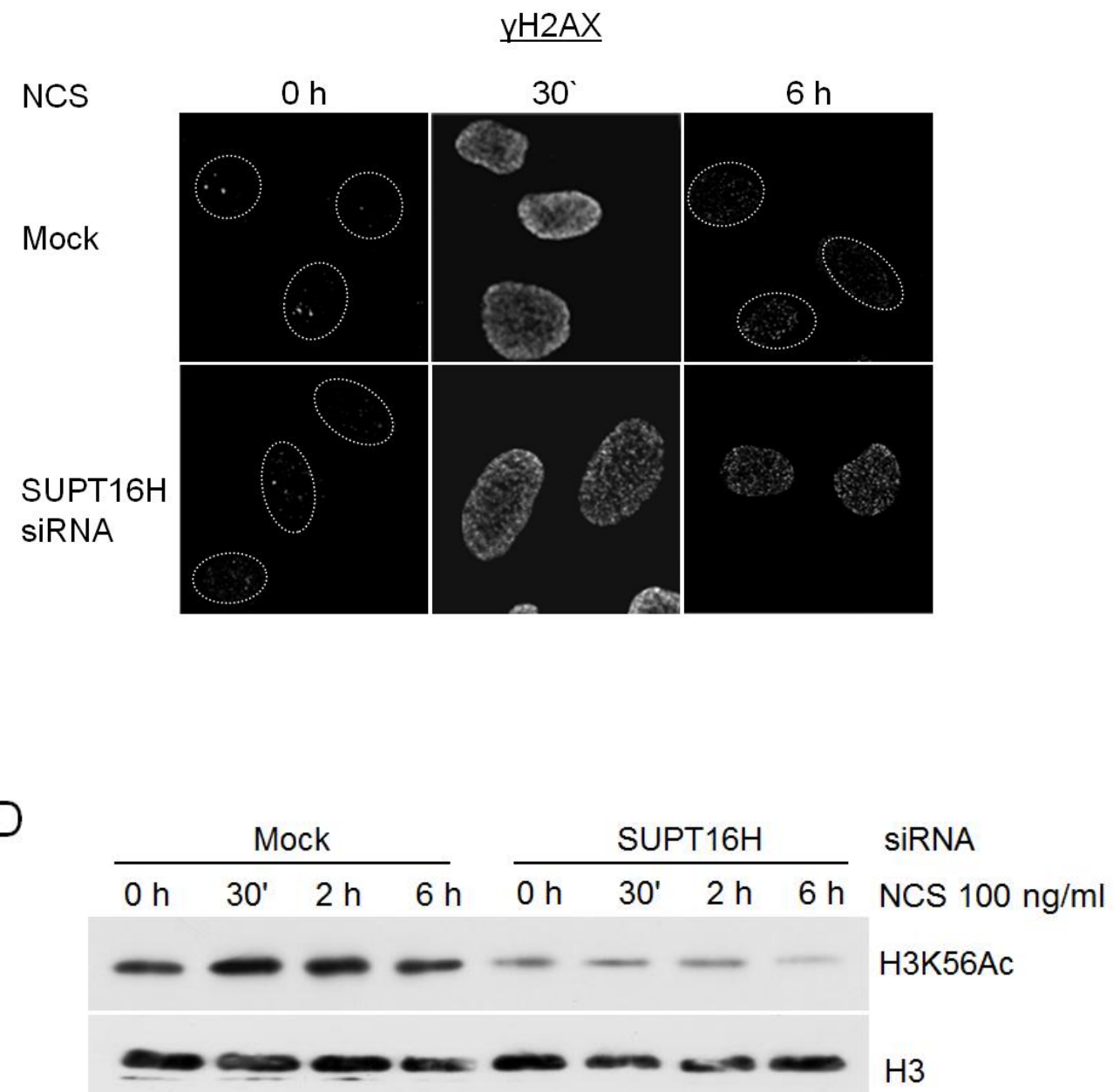




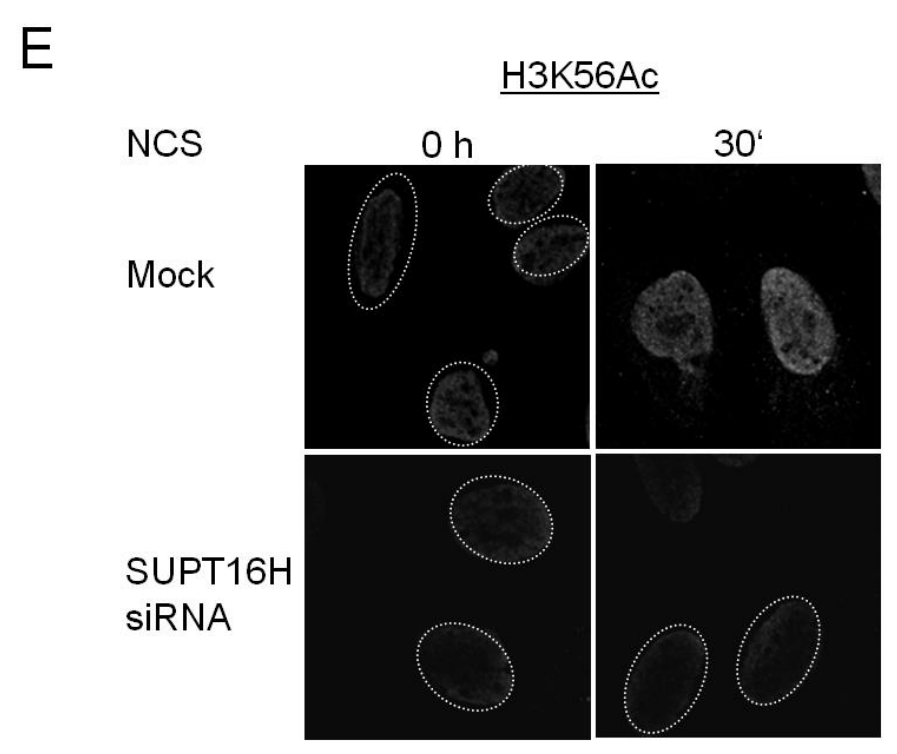

Fig II.3: RNF40 knockdown decreases the binding of SUPT16H and SSRP1 to chromatin. (A) U2OS cells were transfected with RNF40 siRNA or mock for 48 hours and treated with NCS $100 \mathrm{ng} / \mathrm{ml}$ for the indicated times. Chromatin fractions were prepared and analysed by Western blot for SUPT16H, SSRP1 and H2B. (B) SUPT16H depletion phenocopies the effects of RNF40. Knockdown of SUPT16H led to prolonged $\mathrm{YH} 2 \mathrm{AX}$, and decreases in $\mathrm{H} 3 \mathrm{~K} 56 \mathrm{ac}$ after DSB induction similar to RNF40 knockdown. (B) U2OS cells were transfected with SUPT16H or mock siRNA for 48 hours and treated with 100 $\mathrm{ng} / \mathrm{ml} \mathrm{NCS}$ for the indicated times. Chromatin fractions were analysed by Western blot for $\mathrm{\gamma H} 2 \mathrm{AX}$ and H2B. (C) U2OS cells transfected and treated similar to (B) for indicated time points and immunostained for $\mathrm{YH} 2 \mathrm{AX}$. (D) Whole cells lysates from (B) were immunoblotted for H3K56ac and H3. (E) U2OS cells were transfected and treated with NCS similar to (B) and immunostained for H3K56ac after the indicated times.

\section{RNF40 and SUPT16H are required for DNA repair}

One of the essential steps in both the homologous recombination and nonhomologous end joining pathways of DNA repair is the resection of the ends of the damaged DNA (Ciccia and Elledge, 2010). In order for DNA end resection to occur, dynamic changes in chromatin structure are required to make the damaged regions accessible to the DNA repair proteins (Ransom et al., 2010). DSB end resection results in the production of singlestranded DNA (sSDNA) which becomes bound by the sSDNA-binding proteins RPA1 and RAD51. Therefore, based on the known histone chaperone function of SUPT16H and the effects of RNF40 and SUPT16H knockdown on H3K56ac we investigated whether RNF40 and SUPT16H knockdown may also affect DSB end resection. Indeed, while the binding of RPA1 and RAD51 to chromatin increased following the induction of DSBs in control cells, the knockdown of either RNF40 (Fig II.4A) or SUPT16H (Fig II.4B) resulted in decreased RPA1 and 
RAD51 recruitment to chromatin. Furthermore, the knockdown of either also resulted in decreased formation of discrete DSB-induced RPA1 foci (Fig II.4C and D; Supp Table II.1). The formation of DNA DSBs can be visualized using the neutral comet assay. Based on the sustained induction of $\mathrm{YH} 2 \mathrm{AX}$, decreased recruitment of RPA1 and RAD51 to chromatin, and decreased RPA1 focus formation following RNF40 and SUPT16 knockdown, we hypothesized that knockdown of either protein may result in decreased DNA repair. Therefore we performed neutral comet assays in control cells or cells in which RNF40 or SUPT16H were knocked down. In control cells, DNA damage was clearly present based on the formation of comets at both 30 minutes and 2 hours following NCS treatment but returned to nearly basal levels at 6 hours after treatment (Fig II.4E). In contrast, comets were still clearly visible in both RNF40 and SUPT16H-depleted cells 6 hours after the induction of DSB. Therefore, we conclude that both proteins are essential for proper DNA DSB repair.

A

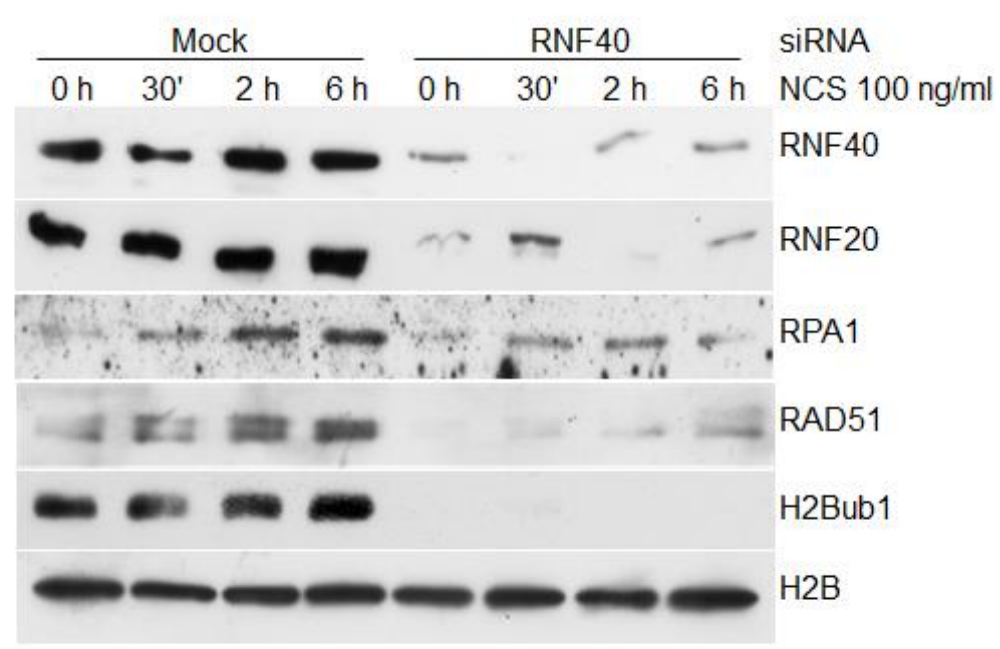

B

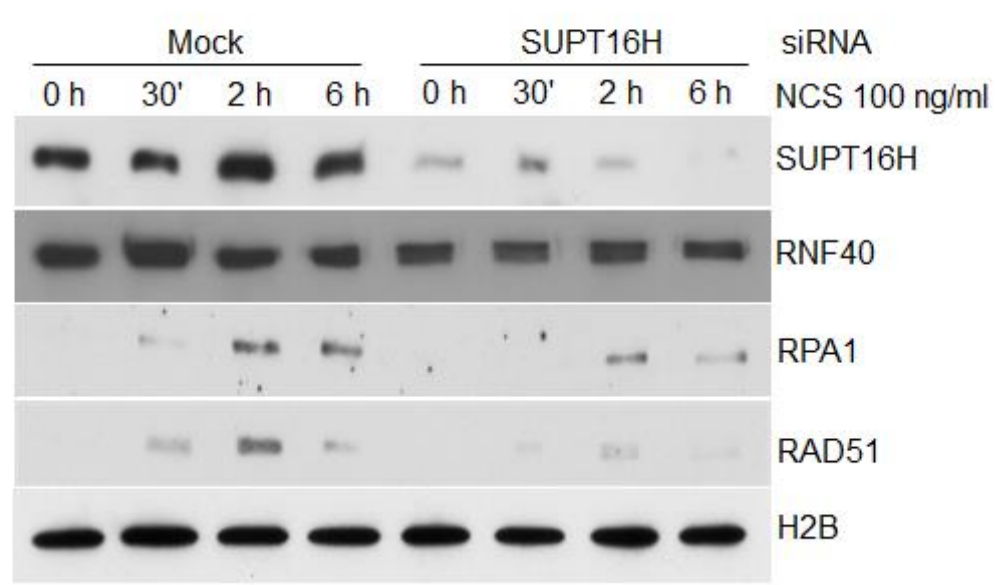


C

RPA1

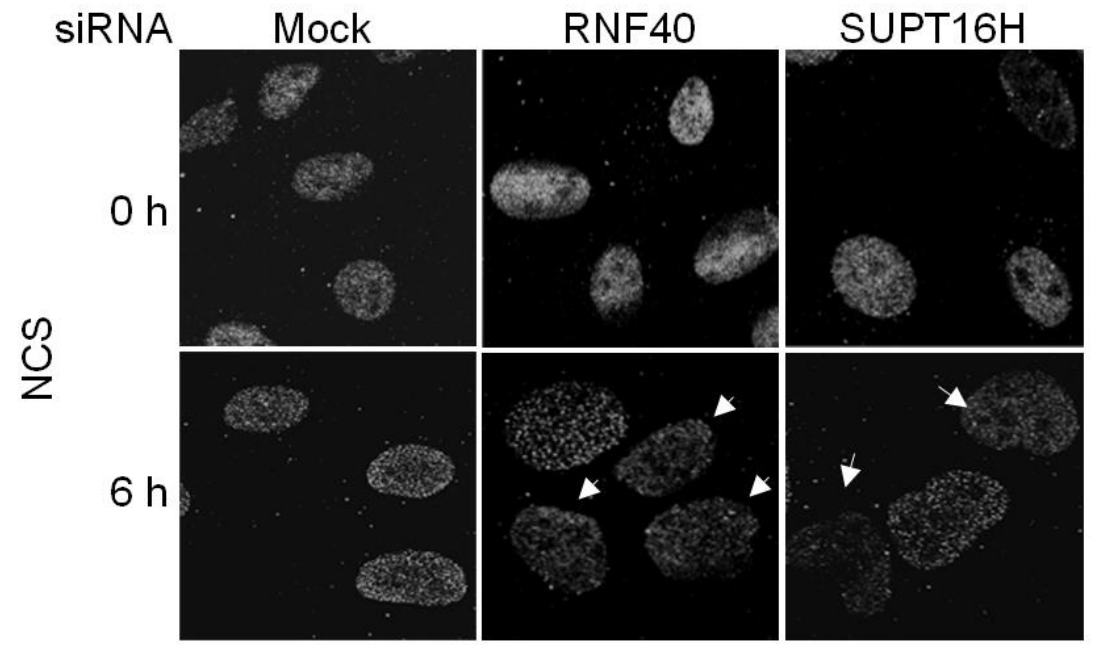

D
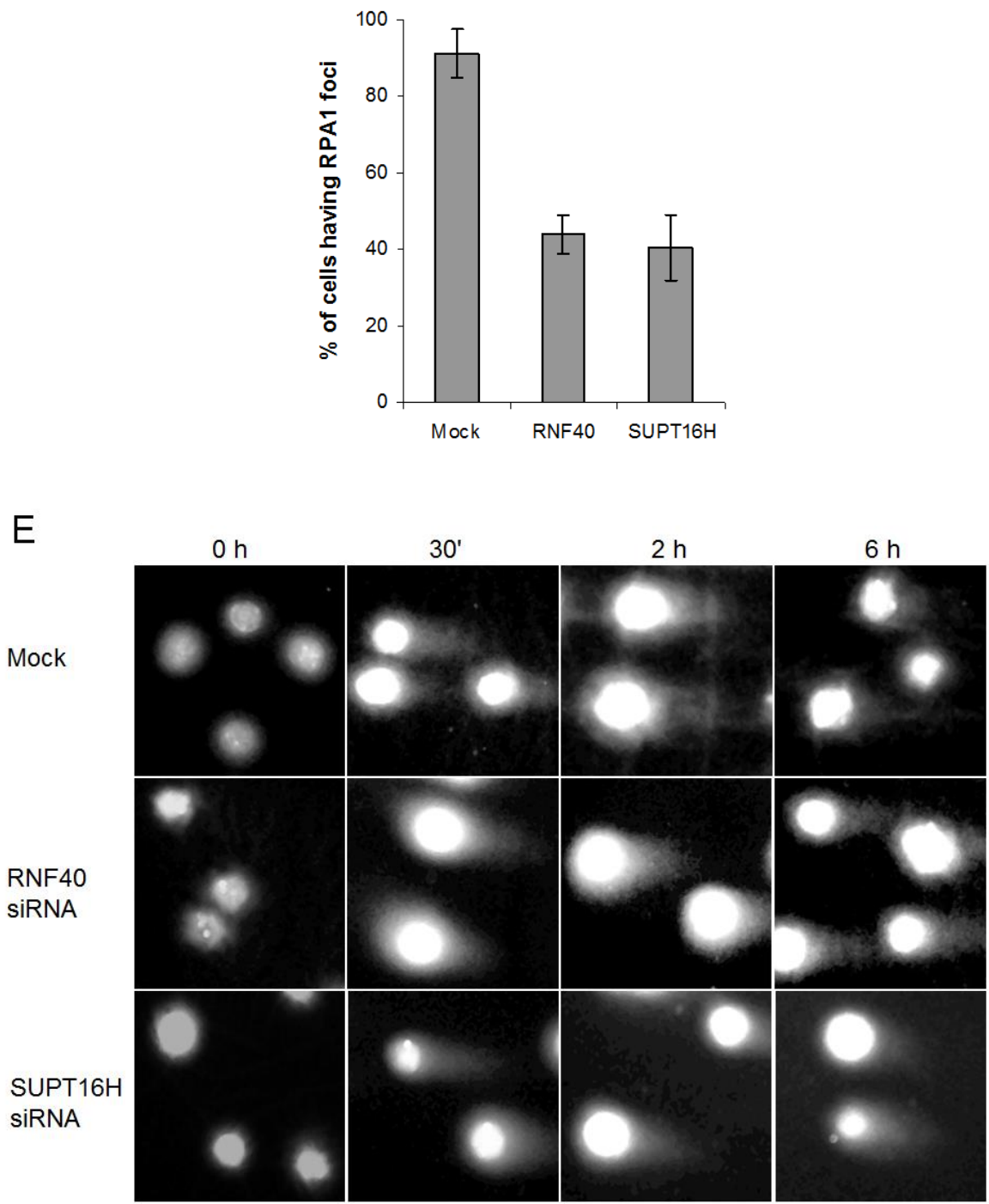
Fig II.4: RNF40 and SUPT16H are essential for RAD51 and RPA1 recruitment to chromatin and DNA repair. U2OS cells were either mock transfected or with siRNAs against RNF40 (A) or SUPT16H (B) for 48 hours and treated with $100 \mathrm{ng} / \mathrm{ml} \mathrm{NCS}$ for the indicated times. Chromatin fractions were prepared and analyzed by Western blot using the indicated antibodies. (C) Loss of RPA1 focus formation following RNF40 or SUPT16H knockdown in U2OS cells upon DSB induction. (D) Graphical representation of the number of cells having RPA1 foci. \% cells with clear foci from the experiment in (C) were counted and plotted on a graph. Four fields, each containing 6-12 cells, were counted per treatment and knockdown. (E) RNF40 and SUPT16H knockdown cells display prolonged DNA damage after 6 hours of NCS treatment. DSBs were observed using the neutral comet assay 48 hours after transfection of U2OS cells with RNF40, SUPT16H siRNA or mock followed by $100 \mathrm{ng} / \mathrm{ml} \mathrm{NCS}$ treatment for the indicated times. Representative images are shown.

\section{Discussion}

The proper regulation of DNA repair plays an essential role in maintaining genome integrity following the induction of DSBs. Dynamic changes in chromatin structure involving changes in histone modifications and the recruitment of specific chromatin-associated proteins to sites of DNA damage are required for DNA repair proteins to gain access to damaged sites (Misteli and Soutoglou, 2009; Ransom et al., 2010; Rossetto et al., 2010) and for the activation of DNA damage signaling (Ayoub et al., 2008, 2009; Ciccia and Elledge, 2010; Sun et al., 2010). Following DNA repair, reassembly of chromatin appears to be necessary for the attenuation of the DDR (Ransom et al., 2010). Thus both chromatin disassembly and reassembly have important consequences for the cellular response to DNA damage. However, the mechanistic role of specific histone modifications, modifying enzymes, histone chaperones and chromatin remodeling enzymes in the DNA damage response and DNA repair remains poorly understood. In this study we sought to determine the role of H2Bub1 in controlling chromatin dynamics during the DNA damage response and DNA repair. Our results suggest that expression of the H2B ubiquitin ligase RNF40 is essential not only for the attenuation of the DNA damage response, but also for the induction of chromatin disassembly and reassembly following DNA DSB. These results are consistent with recent findings where the knockdown of RNF20 resulted in decreased DSBinduced chromatin disassembly (as judged by the induction of non-chromatin bound histone H3 after $\gamma$-irradiation) (Nakamura et al., 2011). Although knockdown of the ATP-dependent Snf2 chromatin remodeling enzyme was shown to elicit similar effects on DNA repair as RNF20 knockdown, whether and how these two pathways are mechanistically connected remains unclear. 
We propose that the FACT histone chaperone complex plays a central role in mediating the effects of H2Bub1 on chromatin dynamics following DSB. This hypothesis was based on the ability of FACT to catalyze the exchange of $\mathrm{\gamma H} 2 \mathrm{AX}$-containing $\mathrm{H} 2 \mathrm{AX}-\mathrm{H} 2 \mathrm{~B}$ dimers in vitro (Heo et al., 2008) as well as the established connection between FACT activity and transcriptional elongation in vitro (Pavri et al., 2006). The functional co-operativity between FACT and H2Bub1 is further supported by their overlapping localization on active genes (Gomes et al., 2006; Minsky et al., 2008) and the common requirement of CDK9 activity for their presence in the transcribed regions of active genes (Gomes et al., 2006; Pirngruber et al., 2009a). However, neither a dependence of FACT on H2Bub1 for its chromatin recruitment, nor a role for FACT in DSB repair has been demonstrated in mammalian cellbased systems. Thus, this study provides the first evidence of a common role for the FACT component SUPT16H and the H2B ubiquitin ligase RNF40 in the induction of chromatin dynamics (as assessed by H3K56ac) and subsequent DNA end resection (based on the impaired recruitment of RAD51 and RPA1). In support of the essential roles of RNF40 and SUPT16H following DSB induction, we observed decreased DNA repair after DSB induction following their depletion.

These results not only provide important insights into the biological function of FACT and $\mathrm{H} 2 \mathrm{Bub1}$ during DNA repair, but they also suggest that H2Bub1 may serve as a diagnostic marker for cancer treatment and/or prognostic marker for patient survival. Chemo- and radiotherapy function to induce cytotoxicity in tumors primarily by causing DNA damage. Treatments such as poly-ADP-ribose polymerase (PARP) inhibitors increase chemo- and radiosensitivity in cancers by interfering with DNA repair (Bryant et al., 2005; Farmer et al., 2005). It is imaginable that new therapies which specifically target H2Bub1 may also be combined with standard radio- or chemotherapies to increase their effectiveness. One such therapeutic target may be CDK9, whose activity we recently showed is essential for the global maintenance of H2Bub1.26 Consistent with this hypothesis, a recent study identified CDK9 in a screen for proteins whose knockdown sensitizes cells to DNA damage ( $\mathrm{Yu}$ and Cortez, 2011; Yu et al., 2010). Another potential target may be the proteasome. Our recent data demonstrated that treatment with the clinically utilized proteasome inhibitor Bortezomib results in decreased H2Bub1 and a concomitant decrease in chromatin-bound FACT (Prenzel et al., 2011). Consistent with the potential utility of H2Bub1-decreasing 
agents in combination with radiotherapy, both Bortezomib and the CDK9 inhibitor flavopiridol significantly increase radiosensitivity in xenograft models (Camphausen et al., 2004; Jung et al., 2003; Pervan et al., 2001; Russo et al., 2001). To what extent decreases in $\mathrm{H} 2 \mathrm{Bub1}$ are involved in these effects will still need to be determined.

Based on these results we propose that the maintenance or loss of H2Bub1 may play an important role in determining both the cellular DDR and DNA repair in response to DSB as well as metastatic potential during tumor progression. Thus H2Bub1 may serve both as a diagnostic marker during tumor progression and a prognostic indicator for tumor responsiveness to radiotherapy. Similar to Metastasis-Associated Protein-1 (MTA1), which probably plays a role in metastasis (Nicolson et al., 2003; Toh et al., 1994) through its interactions with multiple histone modifying enzymes and histone chaperones, a loss of H2Bub1 also correlates with tumor progression and metastasis in breast cancer (Prenzel et al., 2011). Interestingly, MTA1 and RNF40 control estrogen receptor-regulated transcription in opposing manners (Mazumdar et al., 2001; Prenzel et al., 2011). Furthermore, while depletion of MTA1 decreases metastatic properties (Toh et al., 1994), knockdown of either RNF20 or RNF40 increases cellular migration (Prenzel et al., 2011; Shema et al., 2008). These results suggest that MTA1 and RNF20/40 may play opposing roles in metastasis and DNA repair, but whether and how they functionally or physically interact must be clarified. Based on the results presented here, we hypothesize that the loss of H2Bub1 and a subsequent reduction in FACT recruitment to chromatin may represent an important step during tumorigenesis. These changes appear alter both the propensity of a tumor cell to metastasize as well as its responsiveness to chemotherapeutic treatment. In this manner, it may be possible to individualize patient treatment based on the H2Bub1 status of a tumor, so that pathways controlling $\mathrm{H} 2 \mathrm{~B}$ monoubiquitination and deubiquitination may be utilized as therapeutic targets to enhance or induce radiosensitivity in radioresistant tumors. Additional cell-based, xenograft and in vivo transgenic mouse studies will help allow this model to be tested and determine the importance of RNF20, RNF40 and FACT function during tumorigenesis, tumor progression, metastasis and chemotherapeutic responsiveness. 


\section{Materials and Methods}

\section{SiRNAs}

The RNF40 siRNAs used in this study were RNF40 \#1, 5'-GAT GCC AAC TTT AAG CTA ATT-3'; RNF40 \#2, 5'-CAA CGA GTC TCT GCA AGT GTT-3'; RNF40 \#3, 5'-GAG ATG CGC CAC CTG ATT ATT-3'; RNF40 \#4, 5'-GAT CAA GGC CAA CCA GAT TTT-3'\# and were purchased from Dharmacon (Lafayette, CO). These were normally utilized as a SmartPool (Cat. M-00691300) unless otherwise indicated. The siRNA targeting the RNF40 3' untranslated region contained the sequence $5^{\prime}$-GGG CCA ACT TCC AAT CAT TTT- 3' and was purchased from Dharmacon. The SUPT16H siRNA (5'-AAG GAA TTA AGA CAT GGT GTG-3') was purchased from Qiagen (Valencia, CA) and was described previously 55.

\section{Cell culture and transfection}

U2OS cells were grown in high-glucose DMEM (Invitrogen, Carlsbad, CA) containing 10\% bovine growth serum (BGS; Thermo Scientific, Waltham, MA), 1X sodium pyruvate (Invitrogen) and 1X penicillin-streptomycin (Sigma, St. Louis, Missouri). HCT116 cells were grown in McCoy's medium with 10\% FBS and 1X penicillin-streptomycin. For generation of the tetracycline-inducible RNF40 overexpressing cells, the human RNF40 cDNA sequence was amplified by PCR and cloned into the pCDNA4/TO vector (Invitrogen) containing a FLAG epitope tag. The resulting vector was linearized, transfected into a U2OS cell line containing the Tet-repressor 56, and individual clones were selected in medium containing $500 \mathrm{mg} / \mathrm{L}$ Zeocin (InvivoGen, San Diego, CA) and $5 \mathrm{mg} / \mathrm{L}$ of Blasticidin S (Invitrogen). Transfections were carried out using Lipofectamine 2000 or Lipofectamine RNAiMAX (Invitrogen) according to the manufacturer's instructions. Cells were treated with neocarzinostatin (Sigma) as indicated.

\section{Chromatin fractionation, western blots analysis and primary antibodies}

Chromatin fractionation was performed as previously described (Pirngruber et al., 2009a). Protein samples were analyzed by Western blot analysis using the following antibodies: RNF40 (R9029; Sigma); RNF20 (ab33500) and Histone 3 (ab1791) antibodies were purchased from Abcam (Cambridge, England); HSC70 (sc-7298), RAD51 (sc-8349) and SUPT16H (sc28734) antibodies were purchased from Santa Cruz Biotechnology (Santa Cruz, California); 
anti-H2B (07-371), H2Bub1 (05-1312), үH2AX (05-636), H3K56ac (07-677) antibodies were purchased from Millipore (Billerica, MA); RPA1 (NA13) was from Calbiochem (Darmstadt, Germany); p-CHK2 (Thr68) (2661) was purchased from Cell Signaling Technology (Danvers, MA); the SSRP1 antibody (609801) was purchased from Biolegends (San Diego, California); and the anti-BrdU antibody (556028) was purchased from BD Biosciences (Franklin Lakes, N.J.).

\section{Immunohistochemical staining}

Cells were fixed for immunohistochemical staining in PBS containing 4\% paraformaldehyde and permeabilized with $0.1 \%$ Triton X-100. After blocking with PBS containing $10 \%$ serum, cells were incubated in the same buffer with the appropriate primary antibodies (see above) followed by incubation with Alexa-488 (A21202) or Alexa-594 (A11012) conjugated secondary antibodies (Invitrogen, Oregon, USA). Images were captured using a LSM 510 META confocal microscope (Zeiss, Oberkirchen, Germany) and analyzed using the LSM Image Browser (Zeiss).

\section{Flow cytometry}

U2OS and HCT116 cells were grown and transfected with siRNA as indicated. For the analyses of cell cycle checkpoint activation, 48 hours after transfection cells were treated with NCS $100 \mathrm{ng} / \mathrm{ml}$ for 12 hours and labeled with BrdU for the final 1.5 hours and processed as described.

\section{Comet assay}

U2OS cells were transfected with mock or RNF40 or SUPT16H siRNAs and after 48 hours of transfection cells were treated with $100 \mathrm{ng} / \mathrm{ml}$ NCS. The neutral comet assay was performed as described (Tsai et al., 2008). Briefly, cells were harvested and mixed with low-melting agarose and lysed with lysis buffer. Electrophoresis was performed at $1 \mathrm{~V} / \mathrm{cm}$ for 20 minutes in neutral buffer. After staining the slides with propidium iodide for 10 minutes, images were captured by using a Zeiss LSM 510 META confocal microscope. 


\section{Supplementary Figures}

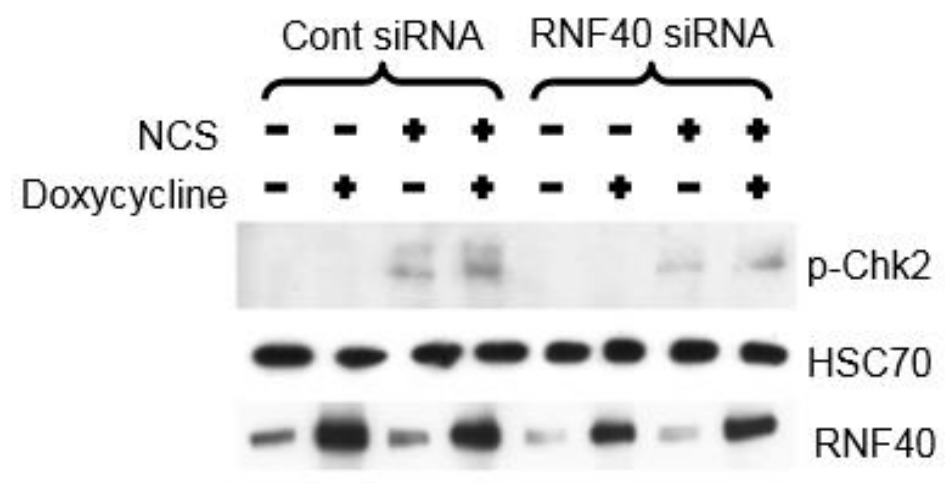

Supp Fig II.1: Loss of CHK2 activation can be partially rescued by overexpression of Flag-RNF40 in U2OS cells. Tetracycline-inducible Flag-RNF40 U2OS cells were transfected with siRNAs targeting the 3' UTR of endogenous RNF40. After 24 hours of transfection, cells were treated with $1 \mu \mathrm{g} / \mathrm{ml}$ of doxycycline as indicated to induce the expression of Flag-RNF40. After 24 hours cells treated with $100 \mathrm{ng} / \mathrm{ml}$ NCS for 15 minutes as indicated and cell lysates were analyzed by western blot for $\mathrm{p}$ CHK2, H2Bub1, RNF40 and HSC70.

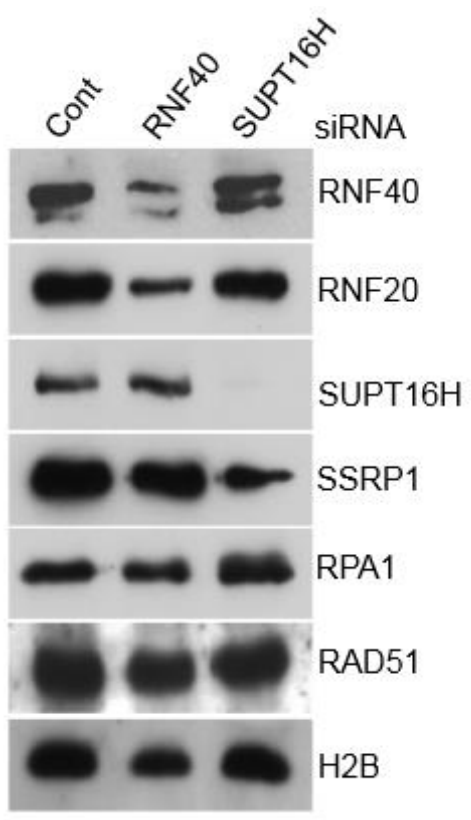

Supp Fig II.2: RNF40 and SUPT16H knockdown do not affect the expression of one another or of the DNA repair proteins RPA1 and RAD51. U2OS cells were transfected with mock or RNF40 or SUPT16H siRNAs for 48 hours and whole cell extracts were analyzed by western blot for RNF40, RNF20, SUPT16H, SSRP1, RPA1, RAD51 and H2B as indicated. 


\title{
2.3 Manuscript in Preparation
}

\section{CHD1 is required for the homologous recombination repair pathway}

\begin{abstract}
Vijayalakshmi Kari ${ }^{1,2}$, Wael Yassin Mansour ${ }^{3^{*}}$, Sanjay Kumar Raul ${ }^{1^{*}}$, Andreas Mund ${ }^{4}$, Simon Baumgart $^{1}$, Hüseyin Sirma ${ }^{5}$, Guido Sauter ${ }^{5}$, Ekkehard Dikomey ${ }^{3}$, Hans Will ${ }^{4}$, Steven A. Johnsen ${ }^{1,2}$

${ }^{1}$ Department of Tumor Biology, University Medical Center Hamburg-Eppendorf, Hamburg, 20246 Germany; ${ }^{2}$ Institute of Molecular Oncology, Göttingen Center for Molecular Biosciences, University Medical Center Göttingen, 37077, Germany; ${ }^{3}$ Laboratory of Radiobiology and Experimental Radiooncology, University Medical Center Hamburg-Eppendorf, Hamburg, 20246, Germany; ${ }^{4}$ Heinrich-Pette-Institute, Leibniz-Institute for Experimental Virology, Department of General Virology, Martinistrasse 52, Hamburg, 20251, Germany; ${ }^{5}$ Institute of Pathology, University Medical Center Hamburg-Eppendorf, Hamburg, 20249, Germany; ${ }^{*}$ Equal contribution
\end{abstract}

Correspondence:

Prof. Dr. Steven A. Johnsen

Department of Tumor Biology

University Medical Center Hamburg-Eppendorf

Center of Experimental Medicine

20246, Hamburg

Germany.

E-mail: sjohnsen@alumni.mayo.edu

Tel.: +49 $407410-57495$

Fax: +49 $407410-55379$

Key words: CHD1, Double strand break, DNA repair, Homologous Recombination (HR)

\section{Own contribution}

Preparation and accomplishment of the biological experiments presented in Fig III.1, Fig III.2, Fig III.3, Fig III.4, Fig III.6 and Fig III.7 i.e cell culture, NCS treatment, chromatin fractionation, western blot, immunofluorescence. Complete figure layout and writing manuscript. 


\section{Abstract}

Chromodomain helicase DNA-binding protein 1 (CHD1) belongs to the ATPdependent chromatin remodeling enzymes implicated in many biological functions including transcription, nucleosome positioning and maintaining an open chromatin status. Recent studies showed that the CHD1 gene is frequently deleted in prostate cancer. Here we examined the role of CHD1 in the DNA double strand break repair pathway in prostate cancer cells. We show that CHD1 is recruited to DNA double strand break sites and is important for cell cycle checkpoint activation. Further, we show that CHD1 depletion decreases CtIP binding to chromatin and end resection upon double strand breaks. Loss of CHD1 specifically affects homologous recombination-mediated DNA repair but not nonhomologous end joining and sensitizes cells to Mitomycin C treatment. Altogether, for the first time we provide evidence for the role of CHD1 in repair of DNA double strand breaks and possible explanation for its tumor-suppressive role by maintaining genomic stability.

\section{Introduction}

The eukaryotic genome is compacted into chromatin composed of DNA, histones and other proteins that regulate chromatin-associated processes including DNA replication, transcription and repair (Kornberg and Lorch, 1999). Most of the DNA-associated processes requires unwinding of chromatin to get access to the DNA where by post-translational histone modifications and chromatin modifiers play key regulatory roles (Jenuwein and Allis, 2001; Strahl and Allis, 2000). DNA damage occurs by both endogenous processes and exogenous agents which trigger the DNA damage response (DDR) in the cell. However, eukaryotic cells have evolved different repair mechanisms to repair different types of breaks at different stages of the cell cycle (Bont and Larebeke, 2004; Shiloh, 2003). Among the different types of DNA damage, DNA double strand breaks (DSBs) are the most common cause of genomic instability and tumor formation. Eukaryotic cells repair DSBs mainly by two different mechanisms including an error prone non-homologous end-joining (NHEJ) and high-fidelity homologous recombination repair (HRR), which requires DNA end resection process (Chapman et al., 2012; Critchlow and Jackson, 1998; Sancar et al., 2004). Upon DNA damage, cells activate DNA damage response (DDR) pathway to induce cell cycle arrest and undergo a cascade of events which allows cells to repair the breaks. DDR pathway involves a 
number of histone modifications which play major roles in recruiting chromatin remodeling and repair proteins. A well characterized histone modification is the phosphorylation of histone variant H2AX at ser139 by ATM, ATR and DNA-PKcs which helps in the further recruitment of DNA damage response mediators and repair proteins such as MRE11/RAD50/NBS1 complex, MDC1, 53BP, CtIP and BRCA1 (Ciccia and Elledge, 2010; Harper and Elledge, 2007). Recent studies on histone chaperone complexes such as CAF-1 and FACT indicate that they also play central role in repair and restoration of chromatin structure after repair (Heo et al., 2008; Kari et al., 2011; Ransom et al., 2010). ATPdependent chromatin remodeling complexes including SWI/SNF, INO80 and ISWI are also involved in the repair of DSBs (Chai et al., 2005; Lans et al., 2012; Morrison et al., 2004; Narlikar et al., 2002; Park et al., 2006). Chromodomain helicase DNA binding protein 1 (CHD1) belongs to the family of ATP-dependent chromatin remodeling factors containing a SNF2-like helicase domain which was shown to recognize and binds to H3K4me2 or me3 through its two chromodomains in humans (Delmas et al., 1993; Lusser et al., 2005; Sims et al., 2005). Studies in Drosophila, yeast and humans have shown that CHD1 is associated with decondensed chromatin (Stokes and Perry, 1995) and interacts with SSRP1, a subunit of the Facilitates Chromatin Transcription (FACT) complex as well as Rtf1 and Spt5 (Kelley et al., 1999; Simic et al., 2003) and maintains chromatin structure during transcription (Smolle et al., 2012). Recent studies have revealed another important function of Chd1 in the positioning of nucleosomes and repression of cryptic transcription in yeast (Gkikopoulos et al., 2011; Hennig et al., 2012; Pointner et al., 2012). Another study has also implicated Chd1 in maintaining the open chromatin status in pluripotent mouse embryonic stem cells with down-regulation of Chd1 leading to accumulation of heterochromatin (Gaspar-Maia et al., 2009). More importantly, CHD1 is the second most deleted or mutated gene in prostate cancer and has been implicated in cancer invasiveness (Burkhardt et al., 2013; Grasso et al., 2012; Huang et al., 2012).

A number of studies have investigated the function of CHD1 in transcription and nucleosome positioning. In the present study, for the first time we show that CHD1 is recruited to DNA DSB site and is required for chromatin remodeling at the site of DNA damage. The loss of CHD1 affects the end resection process by inhibiting the recruitment of RAD50 and CtIP thereby resulting in decreased generation of single strand DNA (sSDNA) as 
indicated by decreased binding of RPA1 and RAD51 to chromatin. Importantly, depletion of CHD1 only affects the homologous recombination mediated DNA DSB repair process but not the non-homologous end joining pathway. Consistently, CHD1 depleted cells are hypersensitive to Mitomycin $\mathrm{C}(\mathrm{MMC})$ treatment which primarily induces beaks which are repaired by HR pathway.

\section{Results}

\section{CHD1 is recruited to the DNA double strand break site}

Most proteins involved in the DNA damage response and DSB repair are recruited to the chromatin, accumulate at the DNA damage site and form foci in the nucleus. We sought to analyze whether CHD1 is also recruited to chromatin and forms foci at the site of DNA damage. In order to do so, we utilized different methods. First we used U2OS19 ptight13 GFP-LacR cell line which has stably integrated I-Scel cleavage site in the genome and the cleavage site is flanked by repeats of lac operator (lacO) at one side and tetracycline responsive element on the other side (tetO). In addition, these cells constitutively express a GFP-lac repressor fusion protein and doxycycline (doxy), inducible expression of the I-Scel endonuclease. Upon doxy treatment I-Scel site cleaved into a DSB and can be visualized by binding of GFP-lac repressor to lacO (Lemaître et al., 2012; Mund et al., 2012). Immunofluorescence studies using U2OS19 ptight13 cells demonstrated that CHD1 is colocalized with GFP-LacR and phosphorylation of $\mathrm{H} 2 \mathrm{AX}$ variant at ser139 ( $\mathrm{H} 2 \mathrm{AX})$ which is a well-known histone modification that occurs at the site of DNA damage within $16 \mathrm{~h}$ of I-Scel induction (Fig. III.1A). The CHD1/GFP-LacR/ $\mathrm{HH}_{2} \mathrm{AX}$ co-localization is not observed in the case of uninduced cells (-I-Sce1) where the I-Scel site is not cleaved. 53BP1 was used as a further positive control which co-localizes with $\mathrm{yH} 2 \mathrm{AX}$ in response to DNA damage (Fig. III.1B).

Further, we could also show that CHD1 is recruited to the sites of DNA DSBs which are induced by the radiomimetic drug Neocarzinostatin (NCS). CHD1 is associated with DNA damage sites or form foci within $4 \mathrm{~h}$ of treatment. Moreover, CHD1 is also associated with the foci which show slower repair kinetics (Goodarzi et al., 2010). Suggesting that CHD1 is recruited to the DNA damage sites in both euchromatic and heterochromatic regions (Fig. 2). To check the kinetics of CHD1 binding to chromatin, chromatin fractionations were prepared from different cell lines which were treated with NCS for different time points. 
The fractions were analyzed by Western blot for CHD1 recruitment to chromatin. In all three cell lines studied including PC3 (Fig. 3A), VCaP (Fig. III.3B) and U2OS cells (Fig. 3C), it is clear that the binding of CHD1 to chromatin is increased in response to DNA DSBs. The binding of CHD1 reaches a maximum at the $6 \mathrm{~h}$ time point (Fig. III.3) and this binding is abolished when CHD1 was depleted either by shRNA or siRNA knockdown.
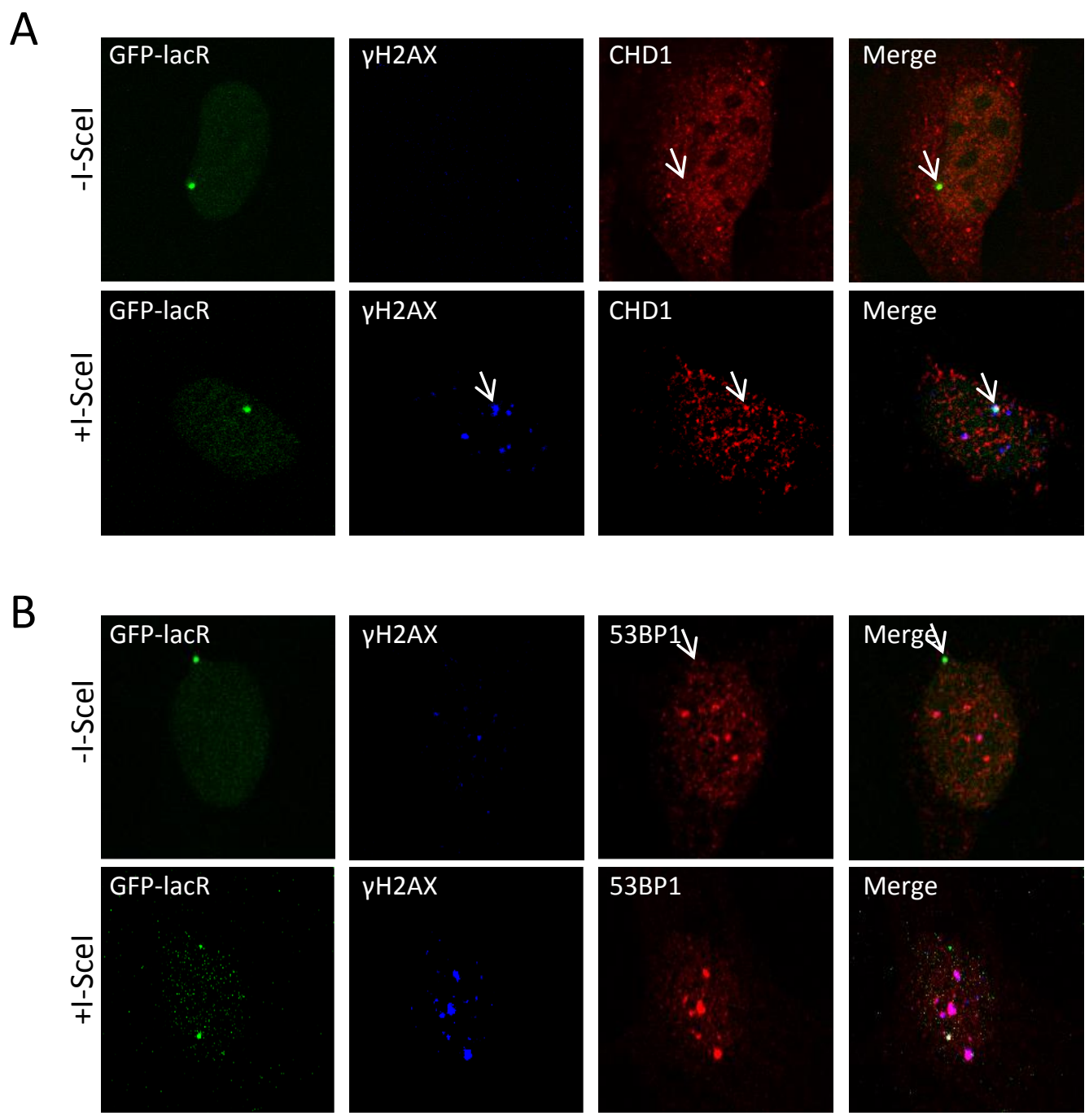

Fig III.1. CHD1 is recruited to I-Scel-induced DSB site and is co-localized with VH2AX. (A) Immunofluorescence studies using U2OS19 ptight13 GFP-LacR cells which have a stably integrated IScel cleavage site flanked by 256 copies of lac operator (lacO) on one side and 96 copies of the tetracycline response element on the other side (tetO). The localization of the GFP-lac repressor protein (GFP-LacR) at the lac-operator DNA sequences in the nucleus before (-I-Scel) and $16 \mathrm{~h}$ after I-Scel-induced (+ I-Scel) DSB. To create DSBS, I-Scel expression was induced by Doxycyclin (Dox) treatment for $16 \mathrm{~h}$. After $16 \mathrm{~h}, \mathrm{CHD} 1$ and $\mathrm{yH} 2 \mathrm{AX}$ co-localize at I-Scel cleavage site, along with DNAbound GFP-LacR but not in uninduced cells (-I-Scel). (B) 53BP1 and YH2AX staining of cells before and after doxy treatment was used as control. 


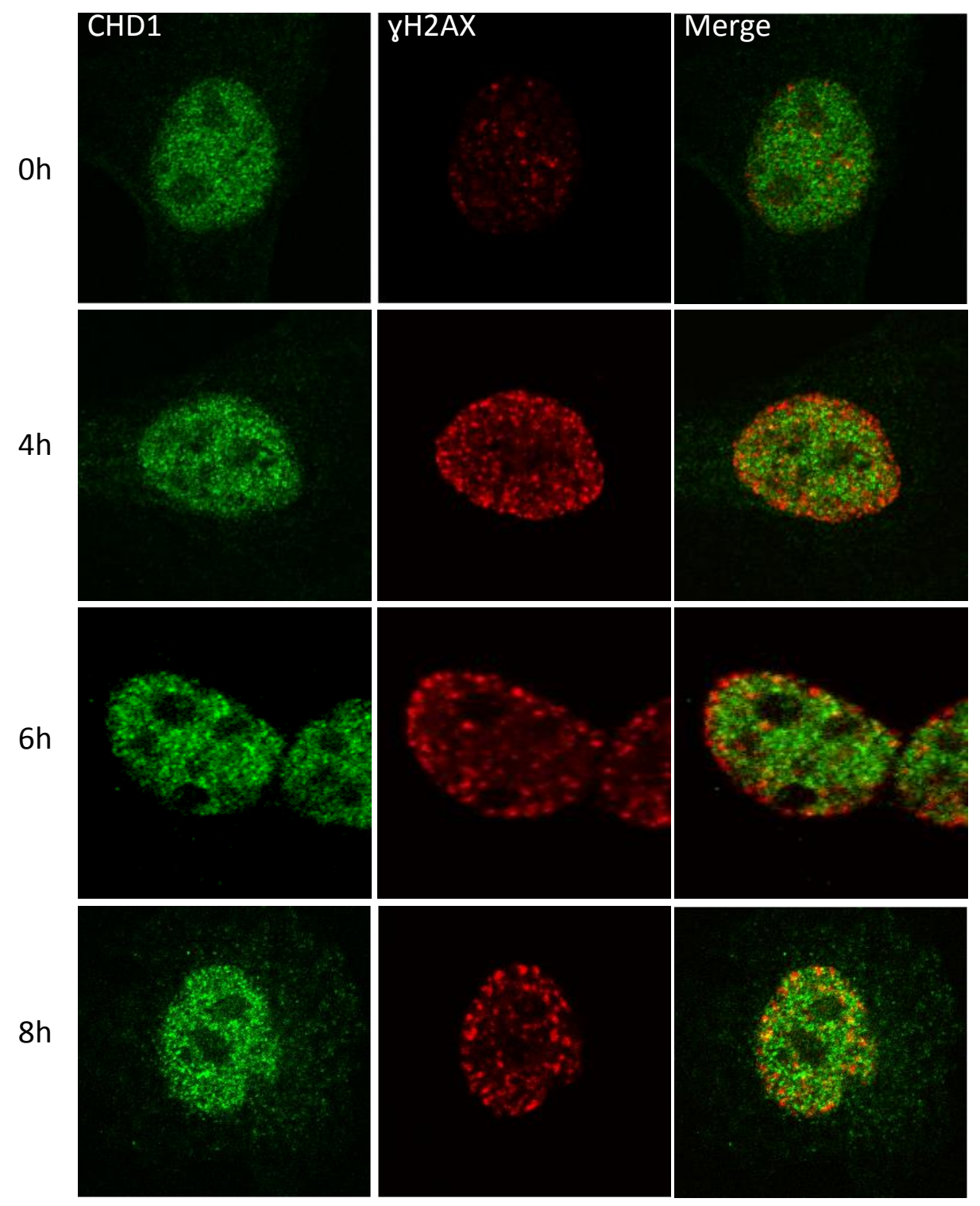

Fig III.2. CHD1 is localized to NCS-induced DNA double strand break sites. Immunofluorescence (IF) studies were performed using PC3 cells to check the co-localization of CHD1 with $\mathrm{YH} 2 \mathrm{AX}$ at DNA damage site. PC3 cells were grown on coverslips and treated with NCS for indicated time points and processed for IF staining as mentioned in methods. CHD1 is stained in green and $\mathrm{\gamma H} 2 \mathrm{AX}$ in red. 

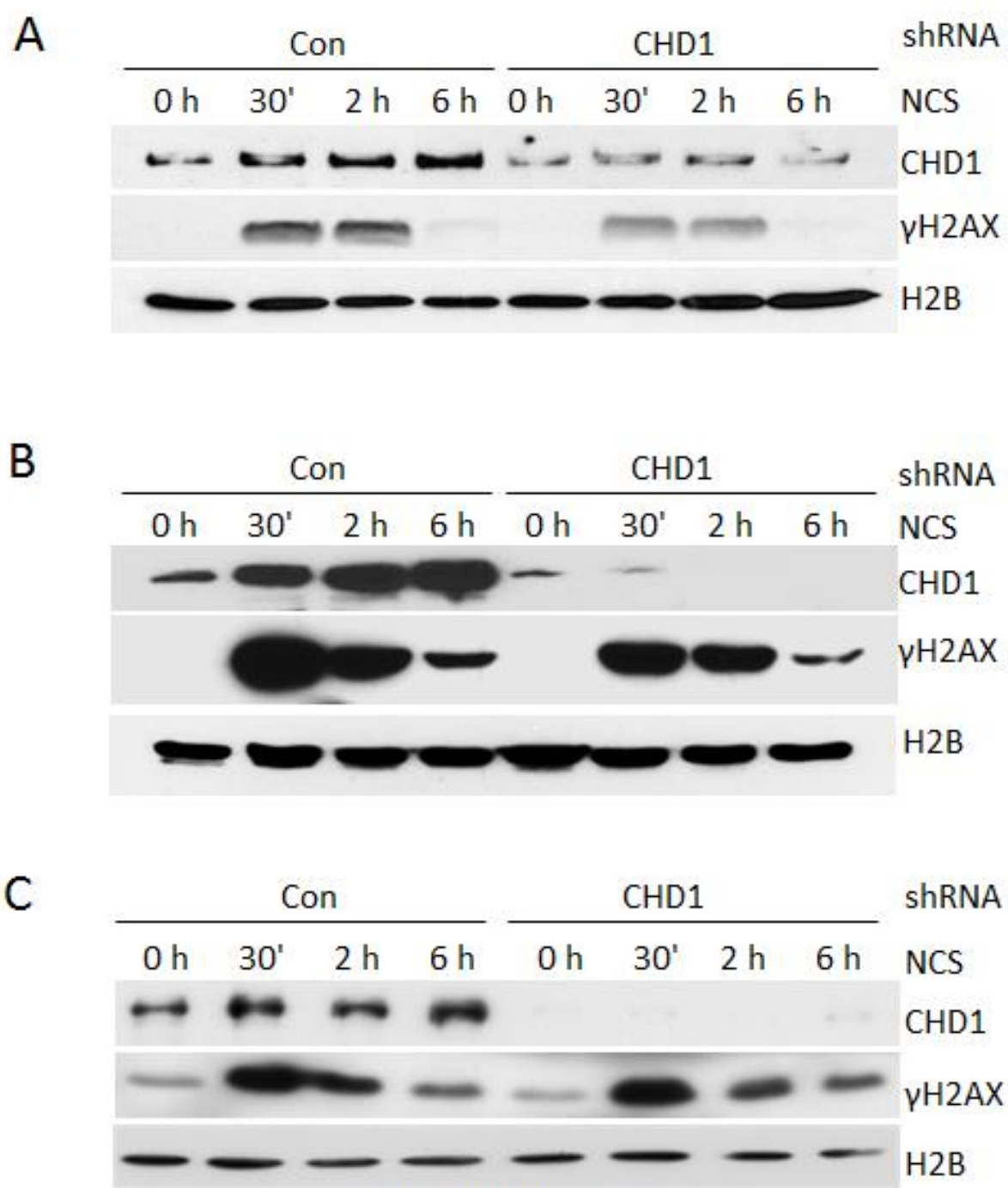

Chromatin fractions

Fig III.3. CHD1 is recruited to the chromatin upon DNA double strand break induction. To look at the recruitment of CHD1 to chromatin upon DNA damage induction, chromatin fractions were analyzed by Western blot at various time points after DSB induction. (A)PC3 cells with stable control and shCHD1 shRNA expression, (B) VCap or (C) U2OS cells transfected either mock or with CHD1 siRNA were treated with NCS for indicated time points and chromatin fractions were immunoblotted with $\mathrm{CHD} 1$ and $\mathrm{\gamma H} 2 \mathrm{AX}$ antibodies, respectively. Whereas, $\mathrm{H} 2 \mathrm{~B}$ detection was used as a loading control.

\section{CHD1 regulates the cell cycle checkpoint activation pathway}

Phosphorylation of $\mathrm{H} 2 \mathrm{AX}$ is the initial step in processing of DNA damage and triggers a cascade of events to repair the breaks (Rogakou et al., 1998b). DNA damage in the cell 
activates checkpoint kinases required for the DNA damage response and arrests cells at specific points in the cell cycle. It is well established that the phosphatidyl inositol-3-kinase (PI3) family member ATM is important for the activation of checkpoint pathway during DNA DSB repair (Lavin, 2008; Shiloh, 2003). ATM is autophosphorylated at different sites in response to DNA damage. A well-studied autophosphorylation site is serine 1981 which is required for further activation and retention of ATM at the damage site (Kozlov et al., 2011). Activated ATM phosphorylates downstream effectors including checkpoint kinase protein 2 (CHK2). We tested whether CHD1 is required for checkpoint activation upon DSB induction. For this purpose, PC3 cells which stably express control or CHD1 shRNA were treated with NCS for $30^{\prime}$ and analyzed by western blot for PATM, PCHK2 and pNBS1. We could show that loss of CHD1 in PC3 cells is deficient in activation of ATM and CHK2 in response to DSB induction (Fig. III.4). The total protein levels of these proteins did not change in CHD1 depleted cells.
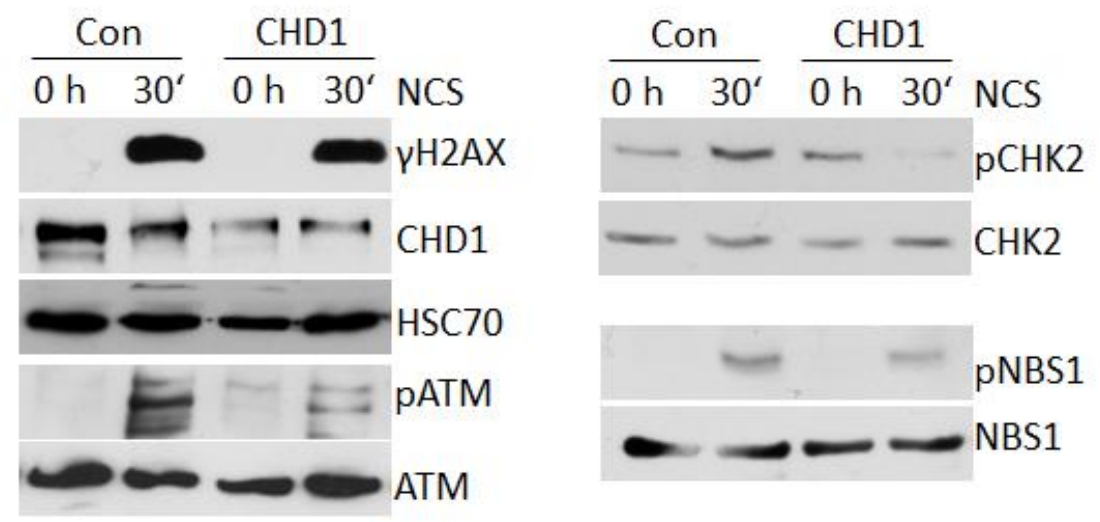

Fig III.4. CHD1 mediates cell cycle checkpoint activation in response to DNA double strand breaks. PC3 cells stably expressing control or CHD1 shRNA were treated with NCS for 30 min and whole cell lysates were analyzed for cell cycle checkpoint activation by Immunoblotting with pATM, pCHK2 and pNBS1 antibodies or antibodies against total protein of the same. The efficiency of knockdown is also shown by western blot against CHD1. HSC70 was used as a loading control.

CHD1-depleted cells show a defect in HR but not in NHEJ repair efficiency and are hypersensitive to Mitomycin c (MMC) treatment

Since CHD1 is recruited to DNA DSB sites, we tested whether CHD1 depletion can affect DSB repair mechanisms including homologous recombination (HR) and nonhomologous end joining (NHEJ) pathways. For this purpose we used well established plasmid based GFP reporter assays. HeLa cells harboring a single copy of HR or NHEJ repair 
substrates were subjected to DSB by transiently expressing the I-Scel endonuclease. The cells were transfected with CHD1 siRNA and repair efficiency was calculated based on the fraction of GFP-positive cells analyzed by flow cytometry. Depletion of CHD1 led to deficiency only in the HR-mediated repair pathway but not in NHEJ (Fig. III.5A-B). As defects in HR pathway in CHD1 depleted cells became apparent, we hypothesized that CHD1 depletion may also affect cell survival after Mitomycin $C$ treatment, which creates DNA breaks that are primarily repaired by the HR pathway. CHD1-depleted PC3 and BHP1 cells treated with different dsoes of MMC displayed significantly decreased survival compared to control cells (Fig. III.5C-D).

A

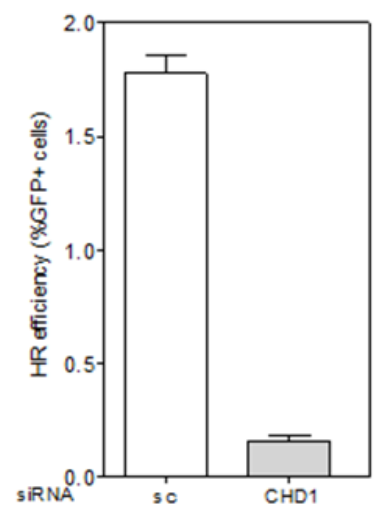

C

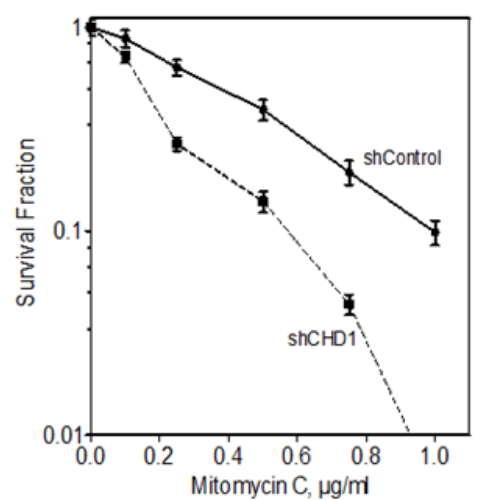

B

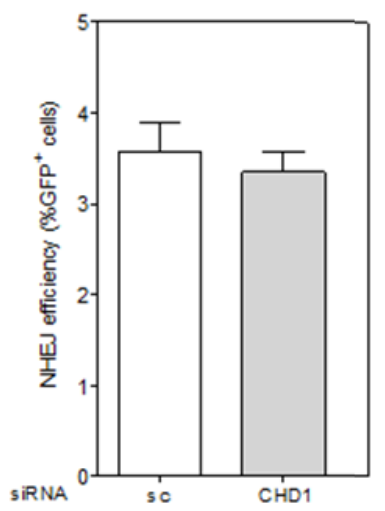

D

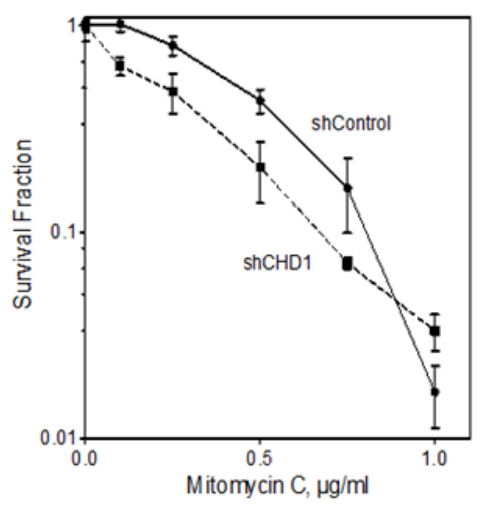

Fig III.5. CHD1 is required for homologous-recombination (HR) repair but not non- homologous end joining (NHEJ) pathway and cell survival after Mitomycin C treatment. (A) HR repair efficiency is decreased in CHD1 depleted cells. (B) NHEJ repair efficiency is not affected by CHD1 depletion. HeLa cells harboring single copies of HR (pGC) or NHEJ (pEJ) repair substrates were either mock transfected or with siRNA targeting CHD1. After $24 \mathrm{~h}$ of transfection DSB was induced by transfecting cells with I-Sce-I-expressing vector (pCMV-I-Scel-3xNLS). After $48 \mathrm{~h}$ of transfection, HR or NHEJ efficiency was calculated based on the fraction of GFP-positive cells. (C) and (D) CHD1-depleted cells show hypersensitivity to Mitomycin $C$ (MMC) treatment. For colony formation assay PC-3 (C) and BHP1 (D) cells which stably express control or CHD1 shRNAs were treated with the indicated doses 
of $\mathrm{MMC}$ for $4 \mathrm{~h}$ and surviving fractions were measured by counting colonies after 3 weeks. The mean values of three independent experiments are shown. Data were normalized to the plating efficiency.

\section{CHD1 is required for the binding of CtIP to chromatin and the end resection process}

To elucidate the function of CHD1 in the repair of DNA DBS through HR mediated repair, we first tested for the effects of CHD1 depletion on DNA end resection process. The resection of ends of the DNA is a key step in the HR process and is mediated by the MRN complex and resulting the recruitment of the $\mathrm{C}$-terminal binding protein-interacting protein (CtIP) (van den Bosch et al., 2003; Sartori et al., 2007; Yu and Chen, 2004). The resulting ssDNA subsequently is bound by the ssDNA binding proteins RAD51 and RPA1 (Symington, 2002).

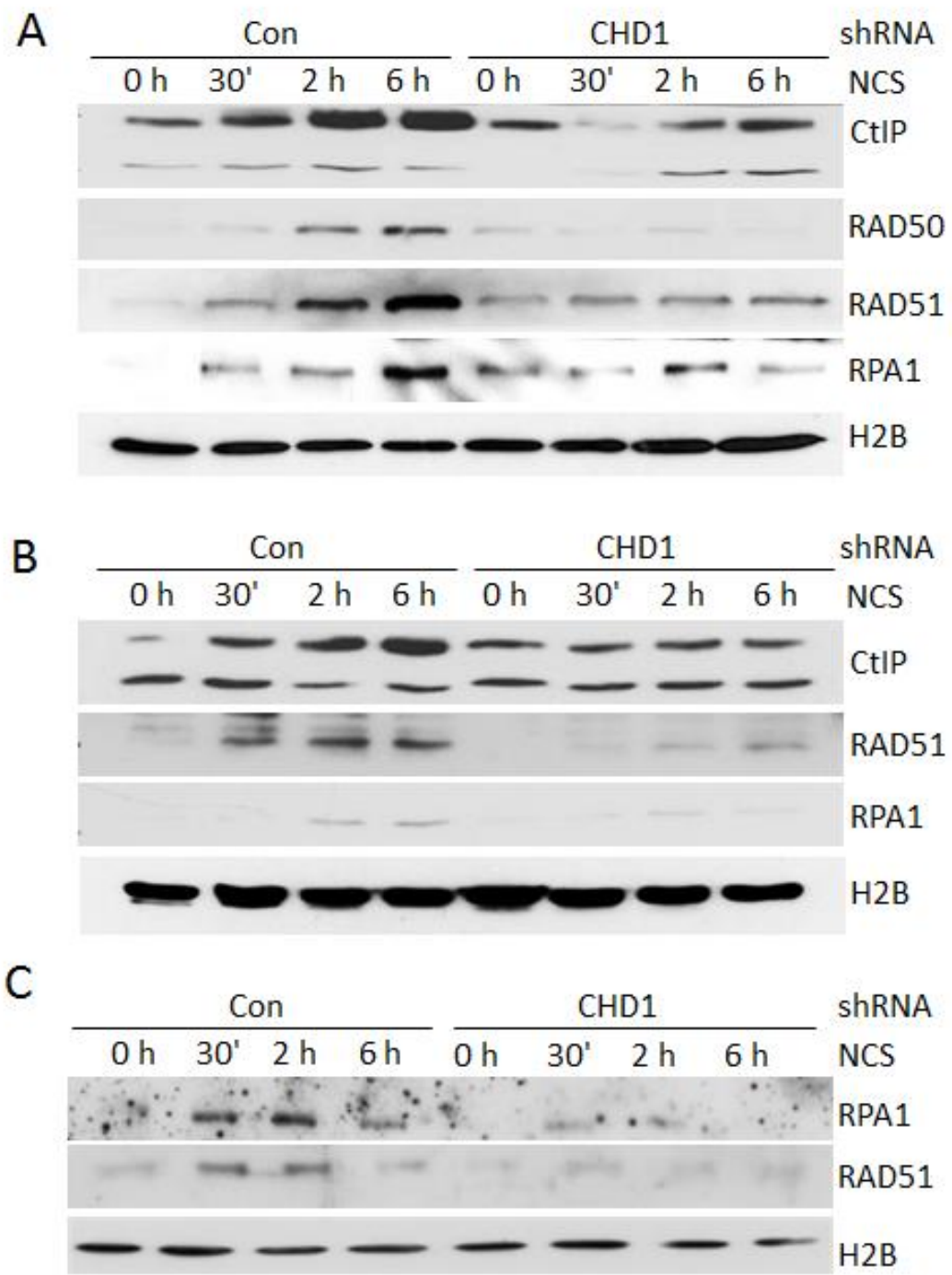

Chromatin fractions 


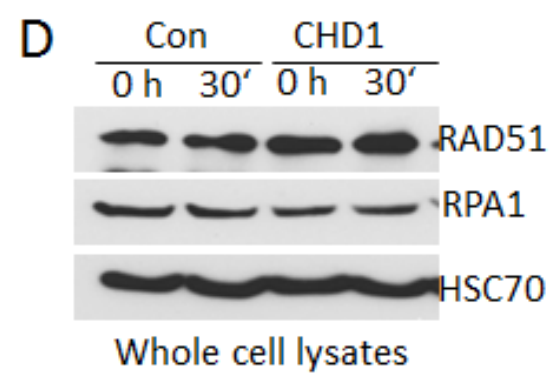

Fig III.6. Depletion of CHD1 affects the end resection and the binding of RPA1 and RAD51 to chromatin in response to DNA DSB induction. (A) PC3 cells with stable expression of either control or CHD1 shRNA were treated with NCS $(100 \mathrm{ng} / \mathrm{ml})$ for indicated time points and chromatin fractions were prepared as mentioned in the methods. Western blot analysis with chromatin fractions for RPA1, RAD51, RAD50 and CtIP are shown. (B) VCap and (C) U2OS cells were transfected either mock or with CHD1 siRNA, after $48 \mathrm{~h}$ of transfection cells were treated with NCS and chromatin fractions were prepared. Western blots showing RPA1 and RAD51 binding to chromatin after DNA damage induction. $\mathrm{H} 2 \mathrm{~B}$ was detected as a loading control.

In order to test the requirement for CHD1 in end resection, PC3 cells stably expressing CHD1 or control shRNA were treated with NCS for different time points and analyzed for the recruitment of CtIP to chromatin. CtIP recruitment to chromatin increased with the time of treatment, but this increase was not observed in CHD1-depleted cells (Fig. III.6A). Based on the decreased recruitment of CtIP CHD1 cells, we further investigated the effects of CHD1 depletion on the recruitment of ssDNA binding proteins RAD51 and RPA1 to chromatin in control and CHD1 depleted cells treated with NCS for different time points. Consistent with the role of CHD1 in end resection western blot data show that CHD1 depletion also affects the binding of RAD51 and RPA1 to chromatin (Fig. III.6A). This clearly suggests that CHD1 controls HR-mediated DSB repair pathway by regulating the binding of CtIP to chromatin. Similar results were obtained with VCaP and U2OS cells where the binding of RPA1 and RAD51 to chromatin was decreased significantly in CHD1 depleted cells in response to DSB induction (Fig. III.6B-C).

Opening of chromatin structure at DSB is required for the repair machinery to gain access to the break sites. Thus we investigated open chromatin status at the DNA damage site using formaldehyde-assisted isolation of regulatory elements (FAIRE) technique in control and CHD1 depleted cells. Specifically AsiSI-U2OS cells which express a tamoxifen inducible AsiSI endonuclease. These cells provide a model in which DSBs can be induced at specific sites in the genome (lacovoni et al., 2010). CHD1 was depleted using siRNA and DNA breaks were induced for $2 \mathrm{~h}$ and $4 \mathrm{~h}$ and then chromatin was analyzed by FAIRE. We 
observed an increased open chromatin status within $2 \mathrm{~h}$ of induction and these changes returned to basal levels in control cells by $4 \mathrm{~h}$ (Fig. III.7). However, CHD1 depleted cells displayed less open chromatin status even without any induction and no increase after the induction of DSB was observed (Fig. III.7).

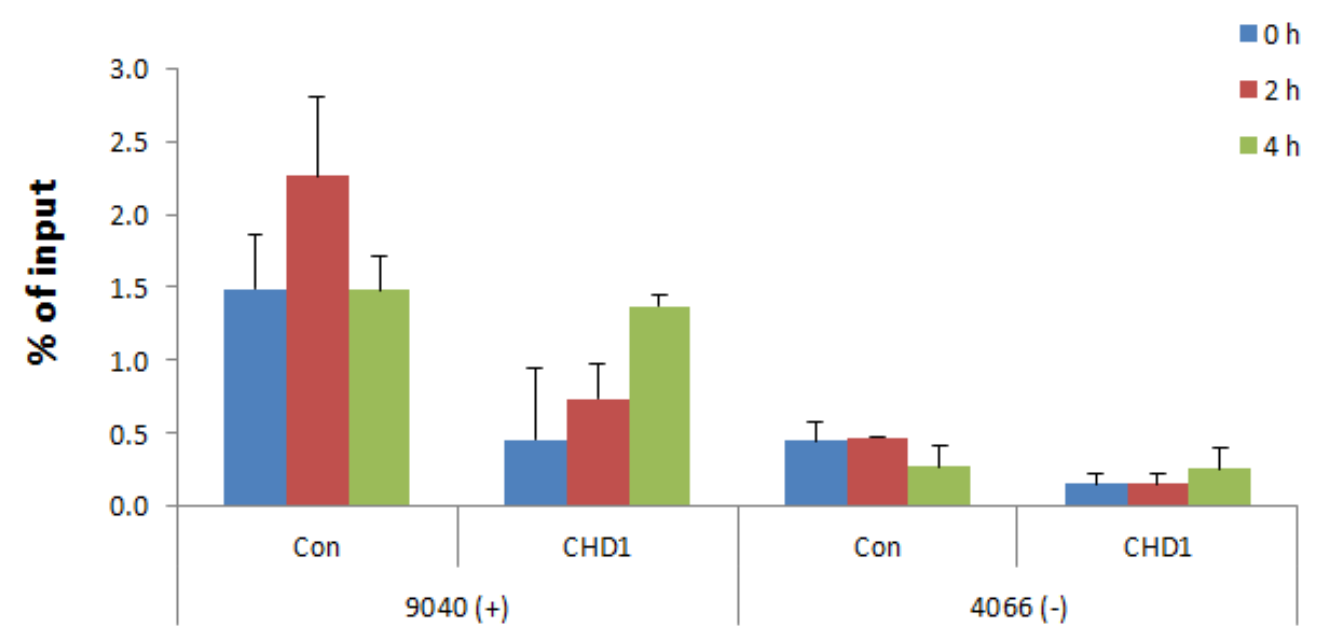

Fig III.7. CHD1 affects end resection process probably through opening up the chromatin at the DNA damage site. Open chromatin status of DNA can be assessed using FAIRE technique. We used U2OS HA-AsiSI-ER cells which express HA- AsiSI restriction enzyme and can create DNA DBSs at specific sites in the genome upon tamoxifen treatment. For FAIRE, U2OS HA-AsiSI-ER cells were mock transfected or with siRNA CHD1, $48 \mathrm{~h}$ of post transfection cells were treated with $300 \mathrm{nM}$ of tamoxifen for $2 \mathrm{~h}$ and $4 \mathrm{~h}$ and process for FAIRE as described in methods.

\section{Discussion}

In this study we provide first the evidence that chromodomain containing DNA helicase 1 (CHD1) is recruited to DNA DSB site and also plays critical role in DSB repair. Moreover, we show that CHD1 is required for the binding of CtIP to chromatin in DNA damage induced cells indicating the role for CHD1 in the end resection process during HRmediated DSB repair.

CHD1 function was described mostly related to transcription and the maintenance of open chromatin status (Gaspar-Maia et al., 2009; Marfella and Imbalzano, 2007). Studies from yeast and Drosophila polytene chromosomes show that CHD1 is associated with promoters and transcribed regions of active genes (McDaniel et al., 2008; Walfridsson et al., 2007). In vitro studies show that CHD1 functions in assembly, remodeling and spacing of nucleosomes (Lusser et al., 2005; Stockdale et al., 2006). A recent study suggested a dependence of H2B monoubiquitination (H2Bub1) is dependent on CHD1 (Lee et al., 2012). 
Our previous study and others have shown that H2B ubiquitin ligase complex containing RNF20 and RNF40 which monoubiquitinates H2B is important for activation of DNA damage response and DSB repair in an ATM-dependent manner (Kari et al., 2011; Moyal et al., 2011; Nakamura et al., 2011). We further showed an intimate connection between H2Bub1 and the histone chaperone complex FACT. Specifically FACT subunit SUPT16H was required for DNA DSB repair and its depletion elicited a similar phenotype to RNF40 knockdown (Kari et al., 2011). Interestingly, CHD1 was also reported to interact with FACT subunit SSRP1 (Kelley et al., 1999) proving further support for the functional interaction of these CHD1, FACT and H2Bub1 in DNA repair. Our result indicates that CHD1 is recruited to DNA DSB site and colocalizes with $\mathrm{yH} 2 \mathrm{AX}$. Similar to most of the proteins involved in the DNA damage response and repair, CHD1 also forms foci at the site of DNA damage following DSB induction. Chromatin fractionation shows that CHD1 recruitment to the chromatin gradually increases in response to DSB induction. The DSB breaks which are present in euchromatin are repaired fast while breaks in heterochromatin are repaired slower. However, it appears that CHD1 is associated with both fast and slow repair kinetics damage sites indicating that CHD1 recruitment is not restricted to euchromatic regions but can also be recruited to heterochromatin-associated DNA damage sites. CHD1 was shown to interact with methylated H3 (H3K4me3) during transcriptional regulation (Sims et al., 2005) it is unclear whether this mechanism is required during DNA repair. However, recent studies show that chromodomain are important for enzyme activity rather than chromatin localization (Hauk et al., 2010). The mechanism of recruitment of CHD1 to DNA damage site still needs to be studied.

Activation of cell cycle checkpoint following DNA DSB induction is an important step to prevent DNA replication prior to repair in order to maintain genomic stability. CHD1 depletion did not affect $\mathrm{H} 2 \mathrm{AX}$ phosphorylation triggered by NCS treatment indicating that CHD1 is not required for the initial recognition of DSBs. However, phosphorylation of CHK2 is significantly decreased in CHD1-depleted cells indicating that the ATM pathway is not fully activated. Plasmid-based HR and NHEJ reporter assays showed that CHD1 depletion affects only HR but not NHEJ suggesting that ATP-depending chromatin remodeling activity of CHD1 is required for HR-mediated DSB repair. One key step in HR is the generation of SSDNA through end resection process which requires the binding of CtIP to the damage site. We 
show that CHD1 depletion leads to reduced recruitment of CtIP to chromatin upon DSB induction. CtIP was initially identified as CtBP interacting protein and interacts with MRN complex and BRCA1 (Sartori et al., 2007; Yu and Chen, 2004). CtIP promotes ATR recruitment in S/G2 phase and end resection process (Sartori et al., 2007). The defects we could see in CHD1 cells on end resection process and ssDNA binding proteins recruitment may be due to decreased recruitment of CtIP to chromatin in response to DSB. CHD1depleted cells show hypersensitivity to MMC treatment where the breaks are converted into DSBs in S phase and are repaired by HR pathway. It suggests that CHD1 is involved in the repair of DSB in S phase through the HR pathway.

CHD1 is deleted in many prostate cancers and loss of CHD1 led to decreased AR binding and its associated ERG rearrangement (Burkhardt et al., 2013). It would be interesting to know whether cancers which have CHD1 deletions are more sensitive to DNA damaging drugs in combination with inhibitors of DNA repair pathway. 


\section{Material and Methods}

\section{Cell culture and siRNA transfection}

PC-3 cells which have stable integration of shControl or shCHD1 were grown in RPMI medium containing $10 \%$ bovine growth serum (BGS; HyClone, USA), 1x penicillinstreptomycin (Sigma, St. Louis, USA) and $1 \mu \mathrm{g} / \mathrm{ml}$ of puromycin (sigma) (Burkhardt et al., 2013). U2OS cells were grown in high-glucose DMEM (Invitrogen) containing $10 \%$ bovine growth serum (HyClone), 1x sodium pyruvate (Invitrogen) and $1 x$ penicillin-streptomycin (Sigma). U2OS19ptight13 GFP-lacR cells were grown in DMEN high glucose, 10 \% BGS, 1x penicillin-streptomycin (Sigma), $800 \mathrm{mg} / \mathrm{ml}$ of G418, and $2 \mathrm{mM}$ IPTG containing medium (Lemaître et al., 2012; Mund et al., 2012) for maintenance. For DNA damage induction cells were treated with doxycycline for $24 \mathrm{~h}$ without IPTG in the medium and processed for immunostaining. AsiSI-U2OS cells were grown in medium containing puromycin and treated with $300 \mathrm{ng} / \mathrm{ml}$ of tamoxifen for indicated time points to induce DSBs as previously described (lacovoni et al., 2010; Massip et al., 2010). To knock down CHD1, siRNA transfections were performed using Lipofectamine RNAiMAX (Invitrogen) according to the manufacturer's instructions. Cells were treated either with Neocarzinostatin (NCS; Sigma) or doxycycline or tamoxifen as indicated. Smart pool of siRNAs used to knockdown CHD1, displayed sequences CAUCAAGCCUCAUCUAAUA; GAUAAGAACUCAUGAAUGG; GAAGAGAGCUGAAACUCAU; GAAACAAGCUCUAGAUCAU;

\section{Chromatin fractionation}

Chromatin fractionation was performed as previously described (Kari et al., 2011). Briefly cells were re-suspended in lysis buffer [10 mM HEPES (pH 7.9), $10 \mathrm{mM} \mathrm{KCl}, 1.5 \mathrm{mM} \mathrm{MgCl}$, $0.34 \mathrm{M}$ sucrose, $10 \%$ glycerol, $0.1 \%$ Triton $\mathrm{X}-100,1 \mathrm{mM}$ dithiothreitol and protease inhibitors] and centrifuged at $1300 \mathrm{~g}$ for $5 \mathrm{~min}$ and nuclear pellet is lysed in nuclear lysis buffer [3 mM EDTA, $0.2 \mathrm{mM}$ EGTA, $1 \mathrm{mM}$ DTT and protease inhibitors] for $30 \mathrm{~min}$ on ice. Soluble chromatin fractions were separated by centrifuging at $1700 \mathrm{~g}$ for $5 \mathrm{~min}$. Chromatin fractions were loaded on SDS -PAGE electrophoresis. Immunoblotting and antibody incubations were performed according to the standard protocols. 


\section{DSB reporter assay}

Double strand break repair efficiency was measured using DSB reporter assay as previously described. Briefly, HeLa cells harboring single copies of HR repair substrate (pGC) or NHEJ repair substrate ( $\mathrm{pEJ}$ ) were transfected with control or CHD1 siRNA. $24 \mathrm{~h}$ after transfection, DSB was induced by transfecting cells with I-Scel-expressing vector (pCMV-I-Scel-3xNLS). Post 48 hours of transfection, percentage of GFP-positive cells were monitored using flow cytometry analysis as an indication for HR and NHEJ efficiency.

\section{Immunofluorescence staining}

Cells were grown on coverslips and treated with NCS $100 \mathrm{ng} / \mathrm{ml}$ or doxycycline for indicated time points. Cells were fixed with $4 \%$ paraformaldehyde for $10 \mathrm{~min}$ and permeabilized with $0.5 \%$ Triton X-100 for 10 min at room temperature. After blocking with $3 \%$ BSA cells were incubated with primary antibodies over night at $4 \mathrm{C}$ and then with fluorescent conjugated secondary antibodies. Images were taken using Zeiss confocal microscope (system).

\section{FAIRE}

Open chromatin status at the DNA damage site is analyzed using FAIRE (Formaldehydeassisted isolation of regulatory elements) in AsiSI-U2OS cells. FAIRE was performed according to the protocol described (Simon et al., 2012). Briefly, $200 \mu$ l of ChIP extracts were used to isolate DNA using phenol:chloroform:isoamyl alcohol mixture and DNA was precipitated using linear polyacrylamide. Extracted DNA was analyzed using qRT-PCR using specific primers listed and $10 \%$ of input is used for normalization.

\section{Colony formation assays}

For colony formation assay PC-3 and BHP1 cells having either control or CHD1 stable shRNA expression were treated with indicated doses of Mitomycin $C(M M C)$ for $4 \mathrm{~h}$ and survival fractions were measured by counting colonies after 3 weeks. The mean values of three independent experiments are shown. Data were normalized to the plating efficiency.

\section{Antibodies}

Antibodies used in the present study are $\mathrm{yH} 2 \mathrm{AX}$ (05-636), CHD1 (06-1339) from Merck Millipore, ATM (A1106) from Sigma, CHD1 (sc-271626), 53BP1 (sc-22760), HSC70 (sc-7298), 
CtIP (Sc-271339) and RAD51 (sc-8349) from Santa Cruz, H2B (Abcam), pCHK2, CHK2, pATM (4526), NBS1 (3002), pNBS1 (3001) and RAD50 (3427) from cell signaling, , RPA1 (NA13) from Calbiochem. 


\section{General Discussion}

\section{Histone mRNA synthesis and 3' end processing}

The core histone proteins are very stable. However when a mammalian cell divides, it must rapidly synthesize a large amount of histones to pack newly synthesized DNA. The synthesis of histones is tightly regulated and starts at the beginning of $\mathrm{S}$ phase. The histone genes that are transcribed during the replication are called replication-dependent histone genes. In most of species including humans, replication-dependent histone genes are clustered together in the genome. Each core histone is encoded by more than one gene. For example, histone $\mathrm{H} 2 \mathrm{~B}$ is encoded by 18 genes. However, all the histone genes are not transcribed at the same efficiency (Fig I.1). The clustering appears to provide a selective advantage for efficient and coordinated transcription and regulation of histone mRNA synthesis (Marzluff et al., 2002). Unlike normal mRNAs, replication-dependent histone mRNAs are not polyadenylated at the $3^{\prime}$ end. Instead they contain a conserved sequence which forms a stem-loop structure (Fig 1) (Marzluff et al., 2008). The unique 3' structure of the histone mRNA is responsible for the recruitment of factors involved in the metabolism and regulation of replication-dependent histone mRNAs. Stem-loop binding protein SLBP recognizes this stem-loop structure and stabilizes the binding of other processing factors at the $3^{\prime}$ end of mRNA (Dominski and Marzluff, 2007).

\section{Polyadenylated histone mRNAs from replication-dependent histone genes and their physiological significance}

mRNAs that are transcribed from replacement histone genes such as $H 2 A X$ and $H 2 A . Z$ which express in the cell cycle phases $\mathrm{GO}$ and $\mathrm{G} 1$ but not in S contain polyA tails at the $3^{\prime}$ end (Mannironi et al., 1989). H2AX protein is synthesized throughout the cell cycle and the ability to produce two different mRNAs may be an advantage for constitutive expression of this variant (Wu and Bonner, 1981b). The mRNAs produced from replication-dependent histone genes are not normally polyadenylated instead they end 3'-terminally with a conserved stem-loop sequence. Interestingly, some replication-dependent histone mRNAs also contain both stem-loop sequence and downstream a polyadenylated site at the $3^{\prime}$ end which suggests that histone mRNAs can be processed by two pathways. A number of studies 
indicate that replication-dependent histone genes produce mRNAs that contain a polyA tail at the $3^{\prime}$ end (Kirsh et al., 1989; Pirngruber et al., 2009a, 2009b; Sullivan et al., 2009; Tan et al., 2013). For example depletion of SLBP, NELF and CDK9 lead to an increased fraction of polyA mRNAs from replication-dependent histone genes. However, the physiological significance of the polyadenylated histone mRNAs that are produced from the replicationdependent histone genes is not clear.

Our study indicates that a subset of histone $\mathrm{H} 2 \mathrm{~B}$ genes is able to produce polyadenylated transcripts upon induction of $\mathrm{G} 1$ cell cycle arrest. The expression of polyA HIST1H2BD and HIST1H2AC were up regulated when cells were exposed to radiation. Moreover, differentiation of hMSCs into the osteoblast and adipocycte lineages led to an increased fraction of polyA transcripts from replication-dependent histone genes. Initially it was thought that polyA mRNAs from replication-dependent histone genes are produced due to misregulation of the $3^{\prime}$ end processing (Pirngruber et al., 2009a; Sullivan et al., 2009). Our study shows that they are produced in response to specific stimuli such as cell cycle arrest, differentiation or DNA damage induction. We could also show that polyA histone mRNAs are transported to the cytoplasm and are found in polysomes. This suggests that cells have evolved an alternative mechanism to produce an extra pool of histones from the same genes when cells are not able to enter into $S$ phase. However, the factors responsible for the synthesis of polyadenylated histone mRNAs from replication-dependent histone genes are not well defined.

Synthesis of polyA histone mRNAs might be an advantage to a non-dividing cell. The polyadenylation at the $3^{\prime}$ end of histone mRNAs may increase the half-life of mRNA and ability to be translated into proteins outside of $S$ phase. We observed an increased fraction of polyA histone mRNAs in the cells that experienced DNA damage. During the repair of DNA damage in the cell, histones are post-translationally modified to sense the DNA damage and repair. Moreover, during the repair of double strand breaks by homologous recombination histones are evicted from the nucleosome in order to generate single strand DNA during the end resection process. To maintain genomic integrity, histones which were removed or lost during the repair of DNA damage need to be replaced with new histones. However, not all the core replication-dependent histone genes express mRNAs that are independent of replication or cell division. Only a subset of histone genes are encoded by replication- 
dependent genes express outside S phase including H3.3, H2A.Z and Macro-H2A (Marzluff et al., 2008). Only few core histones are known to exchange with other histone variants e.g. H3 exchanges with $\mathrm{H} 3.3$ and $\mathrm{H} 2 \mathrm{~A}$ exchange with $\mathrm{H} 2 \mathrm{AX}$. However, core histone proteins $\mathrm{H} 2 \mathrm{~B}$ and $\mathrm{H} 4$ are not known to exchange with any other variants. Hence, increased expression of polyA histone mRNAs from replication-dependent histone mRNAs such as $H 2 B$ and $H 2 A$ may be a mechanism which is adopted to deal with this problem outside of $\mathrm{S}$ phase. Interestingly, loss of core histones is observed in aging cells and aging is also directly correlated with loss of $\mathrm{H} 3$ and $\mathrm{H} 4$. Moreover, overexpressing histones was shown to increase life span in yeast (Feser et al., 2010; Oberdoerffer, 2010). It would be also interesting to study the role of polyadenylated replication-dependent histone mRNAs in cells like neurons or cardiomyocytes which are permanently arrested in G0 or G1 phase.

\section{Post-translational histone modifications and ATP-dependent chromatin remodelers in DNA repair}

We studied the role of one of the PTM histone H2B monoubiquitination at lysine120 (H2Bub1) and its E3 ligase complex RNF20/40 which are associated with actively transcribed genes in DNA damage response and repair. Further, we showed that chromodomain helicase DNA binding protein 1 (CHD1), an ATP-dependent chromatin remodeler is required for the repair of DNA double strand breaks.

\section{Role of RNF20/40 and H2B monoubiquitination in DNA repair}

A number of histone modifications are involved in the DNA repair process starting from the sensing of DNA damage till restoring chromatin structure after the repair. Histones are post-translationally modified (PTM) in response to DNA damage. A well-characterized histone modification is phosphorylation of $\mathrm{H} 2 \mathrm{AX}$ variant at Ser139 position by PI3 kinase family members ATM, ATR and DNA-PKcs. This phosphorylation event is a key step for sensing the DNA damage and recruiting proteins responsible for the chromatin remodeling and repair. However, a number of other histone modifications are also reposted which are required for the DNA repair (Altaf et al., 2007).

H2Bub1 which is associated with actively transcribed chromatin is mediated by RNF20/40 E3 ubiquitin ligase complex. Most of the studies implicated the role of H2Bub1 in 
transcription elongation. H2Bub1 is required for the removal of a repressive mark H3K27me3, from the genes which are required for cell differentiation (Karpiuk et al., 2012). Earlier studies in yeast have shown that $\mathrm{H} 2 \mathrm{Bub} 1$ at lysine 123 mediated by Bre1 is necessary for the cell cycle checkpoint activation (Giannattasio et al., 2005). Recent studies on mammalian H2Bub1 and its E3 ligase complex RNF20/40 suggest that they regulate chromatin structure at the DNA double strand break (DSB) site (Kari et al., 2011; Moyal et al., 2011; Nakamura et al., 2011). The RNF20/40 complex is phosphorylated by a PI3 kinase family member ATM, in response to the DNA damage. RNF20 was shown to interact with NBS1, a component of MRN complex which may be required for its recruitment to the chromatin. H2Bub1 levels were increased in response to DNA damage and this is dependent on ATM. Although it was shown that H2Bub1 levels were increased in response to DSB induction, it is not clear whether $\mathrm{H} 2 \mathrm{~B}$ is monoubiquitinated at the site of DNA damage. Loss of RNF20 was shown to decrease the recruitment of SNF2h, a chromatin remodeling factor and end resection process that generates single strand DNA (ssDNA) for the homologous recombination. On the other hand it is not known whether the defects observed in RNF20/40 depleted cells is due to the loss of its own protein levels or the loss of H2Bub1. There could be a possibility that RNF20/40 may ubiquitinate other proteins or histones which are involved in DNA repair processes. In many DNA associated processes, histone modifications crosstalk with each other to regulate cellular functions. A best example for the histone crosstalk is between $\mathrm{H} 2 \mathrm{Bub} 1$ and $\mathrm{H} 3 \mathrm{~K} 4 \mathrm{me} 3$ during transcription. Crosstalk between histone modifications during DNA repair process is not well characterized. During DNA repair, H2Bub1 may also regulate other histone modifications at the DNA damage site. In yeast, $\mathrm{H} 2 \mathrm{Bub} 1$ is required for H3K79me3 which is methylated by Dot1 and required for the recruitment of 53BP1 protein (Giannattasio et al., 2005; Ng et al., 2002). In humans, regulation of $\mathrm{H} 3 \mathrm{~K} 79$ me3 through $\mathrm{H} 2 \mathrm{Bub} 1$ is not yet investigated.

\section{Role of FACT complex in DNA repair}

The histone chaperone, facilitates chromatin transcription (FACT), a heterodimeric complex comprised of SUPT16H and SSRP1 complex, was initially discovered as to promote RNA Polll associated transcription elongation on the nucleosomal DNA template (Orphanides et al., 1998). FACT complex is associated with the elongating RNA pol II and is proposed to evict nucleosomal histones $(\mathrm{H} 2 \mathrm{~A} / \mathrm{H} 2 \mathrm{~B})$ and deposit them back at the site of 
transcription (Belotserkovskaya et al., 2003). An in vitro study by Heo et al. showed that FACT catalyzes the exchange of $\mathrm{H} 2 \mathrm{AX}-\mathrm{H} 2 \mathrm{~B}$ dimers on nucleosomes. However, the $\mathrm{N}$-terminal domain of Spt16 was shown to bind to H3-H4 but not H2A-H2B in vitro (Stuwe et al., 2008). A recent study showed that Spt16 interacts with $\mathrm{H} 2 \mathrm{~B}$ using its $\mathrm{U}$ - turn motif. This suggests that FACT complex mainly acts as a chaperone for H2A-H2B dimer (Hondele et al., 2013). Interestingly, H2Bub1 functions with the FACT complex to regulate transcription elongation by RNA pol II (Pavri et al., 2006). But, whether there is a physical interaction between $\mathrm{H} 2 \mathrm{Bub1}$ and FACT complex has not been established. In addition to transcription, the FACT complex was also suggested to play role in DNA repair. Studies showed that the FACT complex recognizes Cisplatin-induced DNA adducts and gets recruited to the sites of DNA damage (Sand-Dejmek et al., 2011; Yarnell et al., 2001). However the exact role of the FACT complex in DNA repair remained unexplored. During transcription, H2Bub1 and FACT complex functionally cooperate and appears to be partially responsible for nucleosome eviction. H2Bub1 was shown to be involved in chromatin changes during the repair of DSB and similarly FACT and H2Bub1 might be involved in repair of DNA DSBs.

Our study showed that binding of FACT complex to chromatin was increased in response to DSBs. This increased binding was abolished upon depletion of RNF40 which is responsible for H2Bub1. This suggests that during repair of DSBs, the FACT complex functions in an H2Bub1 dependent manner. Moreover, depletion of RNF40 or SUPT16 decreases the end resection process which requires chromatin remodeling at the DNA damage site. However there is no clear evidence that the recruitment of the FACT complex to chromatin is mediated through interactions with H2Bub1 or the RNF20/40 complex at the DNA damage site. It is also possible that the FACT complex can interact with other histone modifications which occur at the site of DNA damage or with any other proteins which are involved in DNA repair and recruited. Additionally, loss of RNF40 and SUPT16H decrease the efficiency of both HR and NHEJ pathway. A recent study showed that SUPT16H is abundant at somatic hyper mutation (SHM) regions at immunoglobulin genes in the genome and required for histone exchange at the SHM sites (Aida et al., 2013). It will be interesting to determine whether H2Bub1 is also required for the histone exchange at SHM along with SUPT16H. 


\section{The RNF20/40 complex is involved in early or late DNA damage response?}

During the repair of DSBs, it is proposed that chromatin decondensation occurs at two levels, an initial decondensation to open the higher order chromatin structure, and the second decondensation step to remove the rearranged nucleosomes. It was proposed that RNF20/40 is involved in DNA DSB repair pathway at the second level decondensation (Nakamura et al., 2011). On the other hand, our data suggests that RNF40 depletion can block the activation of check point pathway within 15 min of DNA DSB induction. Moreover, increased H3K56ac following DNA damage was not observed in RNF40-depleted cells within 30 min of DSBs. This suggests that RNF20/40 may be required for the initial activation and decondensation steps rather than later steps. The kinetics of DNA repair in both euchromatin and heterochromatin differs due to accessibility of DNA. Since H2Bub1 is known to be associated with active chromatin, it is likely that DNA damage sites which are already occupied by H2Bub1 may be repaired faster compared to the regions with no or less H2Bub1. The role of pre-existing H2Bub1-mediated repair at the damage site for the efficient DNA damage response and repair need to be investigated. It may also be interesting to study whether H2Bub1 similarly increase at the DNA damage sites in heterochromatin regions in comparison to euchromatin regions.

\section{ATP-dependent chromatin remodelers in DNA repair}

A large group of chromatin-remodeling complexes has been identified which are important for controlling gene expression. It has recently also become clear that ATPdependent chromatin remodeling complexes play essential role in modifying chromatin structure during the repair of DNA damage. These complexes disturb DNA-histone interactions using energy from ATP hydrolysis. During the repair of DSB, chromatin remodeling is required either to remove histones or slide them in order to get access to the DNA. Four structurally related ATP-dependent chromatin remodeling complexes have been implicated in DNA repair including SWI/SNF (switching defective/sucrose non-fermenting), INO80 (inositol requiring 80), CHD (chromodomain, helicase, DNA binding) and ISWI (imitation switch) complexes (Lans et al., 2012). In the present study we investigated the role of CHD1 which belongs to the CHD family of ATP-dependent chromatin remodeling complexes in DNA DSB repair pathway. 


\section{CHD1 regulates chromatin structure during DSB repair}

Chromodomain helicase DNA binding protein 1 (CHD1) belongs to the ATPdependent chromatin remodeling factor family which contains a SNF2-like helicase domain which was shown to recognize and bind to H3K4me3 (Delmas et al., 1993; Lusser et al., 2005; Sims et al., 2005). CHD1 is associated with decondensed chromatin and required for transcription. Moreover, CHD1 was shown to regulate positioning of nucleosome and repression of cryptic transcription in yeast (Gkikopoulos et al., 2011; Hennig et al., 2012; Pointner et al., 2012). Importantly, CHD1 was shown to be mutated or deleted in a large fraction of prostate cancers and was implicated in cancer invasiveness (Burkhardt et al., 2013; Grasso et al., 2012; Huang et al., 2012). It was shown that CHD1 is required for efficient recruitment of androgen receptor (AR) to the promoters and expression of ARresponsive tumor suppressor genes. Moreover, loss of CHD1 prevents formation of ERG rearrangements due to impaired AR-dependent transcription which is prerequisite for ERG translocation (Burkhardt et al., 2013). Interestingly, CHD1 was shown to interact with a subunit of FACT complex, SSRP1 (Kelley et al., 1999). Furthermore, one study showed the co-dependency of H2Bub1 and CHD1 in nucleosome reassembly (Lee et al., 2012). Since the FACT complex and H2Bub1 cooperate during the DSB repair process, we hypothesized that CHD1 may also be involved in the repair of DSBs. There are no studies indicating the role of CHD1 in the DNA damage repair process.

In the present study, we showed that CHD1 is recruited to I-Scel-induced DNA damage sites stably integrated into the genome. Further, CHD1 is recruited to DSBs which were created by NCS treatment, but the mode of recruitment is not defined. It is possible that CHD1 may be recruited to the damage site through interaction with the FACT complex. Some studies showed that $\mathrm{H} 3 \mathrm{~K} 4 \mathrm{me} 3$ is increased at the DNA damage site and is dependent on H2Bub1 and RNF20/40 complex. There is a possibility that CHD1 may interact with H3K4me3 at the DSB site in a manner similar to its recruitment during transcription. Since CHD1 is a chromatin remodeling enzyme one could assume that CHD1 is important for the remodeling of chromatin at the DNA damage site. Upon DNA damage, cells activate cell cycle checkpoint kinase pathways to initiate a DNA damage response. The PI3 kinase members ATM, ATR and DNA-PKcs are responsible for activation of the checkpoint kinase pathway. ATM is important for sensing the DNA DSBs and activated ATM phosphorylates a 
number of proteins which are involved in the DNA repair process. We observed that depletion of CHD1 decreases the activation of ATM as well as its downstream target CHK2. This suggests that CHD1 is important for sensing the DNA damage by ATM. CHD1 is probably involved in ATM activation perhaps by changing the chromatin structure at DNA damage site. It is known that alterations in the chromatin structure are sufficient to activate ATM (Kim et al. 2009).

DNA DSBs are repaired mainly by two different pathways known as homologous recombination (HR) and non-homologous end joining pathway (NHEJ). NHEJ pathway is active in all phases of cell cycle and the HR pathway is more favored in S/G2 phase of cell cycle due to the availability of sister chromatid to copy the sequence to repair the DSBs. The HR pathway is faithful compared to NHEJ where the DSBs are repaired by simply joining the ends with little or no homology. At the same time, HR pathway requires extensive chromatin remodeling at the damage site compared to NHEJ. In HR pathway, to copy the genetic information from the sister chromatid, ssDNA is generated from the damaged DNA template by end resection process. A number of proteins are involved in the end resection process including the MRN complex, CtIP and EXO1. The breaks are recognized by the MRN complex which recruits factors responsible for the end resection process including CtIP. The EXO1 processes the end to generate SSDNA. Then the ssDNA is coated with ssDNA binding proteins RPA1 and RAD51 which is then followed by the strand invasion process and synthesis of complementary DNA (Fig 4). ATP-dependent chromatin remodelers play an essential role in the generation of SSDNA by promoting chromatin remodeling and removal of histones at the DNA damage site.

In our study CHD1 depletion showed decreased recruitment of CtIP to chromatin in DNA DSB induced cells. That suggests that CHD1 may work along with the MRN complex to recruit CtIP to chromatin. Consistent with less CtIP recruitment, CHD1-depleted cells show decreased binding of RPA1 and RAD51 binding to chromatin upon DSB induction. Decreased recruitment of CtIP to chromatin mainly affects the end resection process where nucleosomes at the DSB either removed or slide away to generate ssDNA. This clearly indicates that CHD1 promotes end resection process through CtIP recruitment. It is not clear whether the chromatin remodeling activity of CHD1 or the interaction of CHD1 with proteins that recruit CtIP are required for the CtIP recruitment to chromatin. In the HR 
pathway, CHD1 may be involved directly through remodeling nucleosomes at the DSB site but not effect on NHEJ where it does not require extensive chromatin remodeling. Supporting out hypothesis, GFP based reporter assays indicate that CHD1 depleted cells show less efficiency of HR but not NHEJ. This indicates that CHD1 participates in the HR repair pathway probably through its chromatin remodeling activity. Moreover, CHD1 depleted cells are hypersensitive to Mitomycin C (MMC) treatment that creates breaks which can be repaired by HR. All these results made us to understand the role of CHD1 in homologous recombination mediated DNA damage repair.

\section{Are RNF40, FACT and CHD1 in the same axis?}

Our data overall suggests that CHD1 is specifically involved in repair of DSB by the HR pathway. However, the mechanism of CHD1 recruitment to chromatin and how it regulates the recruitment of CtIP is not known. CHD1 interaction with the FACT complex and regulation of $\mathrm{H} 2 \mathrm{Bub} 1$ may explain the mechanism of $\mathrm{CHD} 1$ recruitment to chromatin at DSB sites. The H2Bub1 is implicated in maintaining $\mathrm{H} 3 \mathrm{~K} 4 \mathrm{me} 3$ and recruiting SNF2h chromatin remodeling enzyme to DSB site. Similarly, CHD1 may interact with H3K4me3 in order to be recruited to the DSB. The loss of H2Bub1 by RNF40 depletion may decrease the recruitment of CHD1 to chromatin. Experiments need to be carried out to understand the connection between CHD1, FACT and H2Bub1 in the DNA repair process. Preliminary experiments using HR and NHEJ GFP based reporter assays showed that SUPT16H and RNF40 depletion decreased the efficiency of both HR and NHEJ. However, CHD1 depletion decreased only HR but not NHEJ. This suggests that CHD1 may function downstream of FACT and H2Bub1 in DSB repair pathway. However, we cannot rule out the possibly that FACT and H2Bub1 may also be working together with CHD1 during HR pathway but not in NHEJ. Better understanding the mechanism of this cooperativity could pave way to investigate specific roles of these proteins in HR pathway and repair.

\section{Tumor suppressive role of the RNF20/40 complex}

A previous study demonstrated that, RNF20-depleted cells have higher migration potential compared to the control cells (Shema et al., 2008). RNF40 depletion also shows similar effects suggesting that RNF20/40 complex have tumor suppressive role in the cell (Prenzel et al., 2011). Further, we and others have shown that RNF40 is required for DNA 
DSB repair pathway (Kari et al., 2011; Moyal et al., 2011; Nakamura et al., 2011). Recently, it was shown that RNF20/40 depletion leads to replication stress, formation of R-loops and chromosomal instability (Chernikova and Brown, 2012). Altogether these data suggest that the RNF20/40 complex is important for maintaining genomic stability and suppression of tumor formation.

\section{DNA repair pathway as a target for cancer therapy}

Many chemotherapeutic drugs like Cisplatin, Mitomycin C and 5-Fluorouracil are used in clinic to treat cancers by inducing DNA damage in the cells (Helleday et al., 2008). Cancer cells repair these breaks by different repair mechanism based on type of DNA damage. Using DNA repair inhibitors in combinational therapy approaches could give us better outcome when we use the drugs which induce DNA damage.

CHD1 has been shown to be deleted in many prostate cancers (Burkhardt et al., 2013; Grasso et al., 2012; Huang et al., 2012). Recently it was shown that CHD1 deleted tumors show excess intra-chromosomal rearrangements and gene deletions (Baca et al., 2013). On the other hand loss of CHD1 in prostate cancers led to decreased binding of $A R$ and AR associated gene rearrangements including the TMPRSS:ERG translocation (Burkhardt et al., 2013). Chromosomal translocations occur due to joining of two different chromosomal ends where the NHEJ pathway has been implicated in this process. Decreased translocation in CHD1 depleted cancers could be due to less efficient NHEJ pathway. Interestingly our data suggests that CHD1 deletion decreased the repair of DSBs by HR pathway but not NHEJ. The essential role of CHD1 in translocations is through AR dependent chromosomal interactions and loss of CHD1 led to decrease this interactions and less translocations. There is a possibility that cells lacking CHD1 mainly repair the DSBs by NHEJ which is most likely to create mutations, deletions or translocations and development of tumors.

To check the clinical relevance of CHD1 deletion one could use radiotherapy or chemotherapy to induce DNA damage in the CHD1 deleted cancers and simultaneously inhibit other repair pathways to block completely repair of the DNA damage. For example to block NHEJ pathway, one might use recently developed DNA ligase IV inhibitor in CHD1 deleted tumors (Srivastava et al., 2012). The metastatic castration-resistant prostate 
cancers are treated with Mitoxantone which inhibits type II topoisomerase. While this therapy has mostly been abandoned in the clinic, stratification of patients based upon their CHD1 status may help to identify individuals with castrate-resistant prostate cancer which may have a higher potential to respond Mitoxantone, perhaps in combination with NHEJ inhibitors. There by CHD1 deleted cancers might have significantly improved prognosis when treated with such kinds of combinations of DNA damage inducers and DNA repair inhibitors. In order to test this, xenograft tumor models that are generated from CHD1 depleted cells can treat with DNA damage inducing drugs in combination with DNA reapir inhibitors. It may also be interesting to study the role of CHD1 depletion in other cancers and its clinical application. In addition, using CHD1 inhibitors to block chromodomains or the ATPase activity of CHD1 to treat CHD1 positive tumors in combination with NHEJ inhibitors could inhibit both AR dependent transcription and also HR pathway.

In summary, here we provide an evidence for the role of CHD1 in DSB repair pathway and its tumor suppressor function. 


\section{References}

Abba, M.C., Hu, Y., Sun, H., Drake, J.A., Gaddis, S., Baggerly, K., Sahin, A., and Aldaz, C.M. (2005). Gene expression signature of estrogen receptor alpha status in breast cancer. Bmc Genomics 6, 37.

Ahel, D., Horejsí, Z., Wiechens, N., Polo, S.E., Garcia-Wilson, E., Ahel, I., Flynn, H., Skehel, M., West, S.C., Jackson, S.P., et al. (2009). Poly(ADP-ribose)-dependent regulation of DNA repair by the chromatin remodeling enzyme ALC1. Science 325, 1240-1243.

Aida, M., Hamad, N., Stanlie, A., Begum, N.A., and Honjo, T. (2013). Accumulation of the FACT complex, as well as histone H3.3, serves as a target marker for somatic hypermutation. Proc. Natl. Acad. Sci.

Allan, J., Cowling, G.J., Harborne, N., Cattini, P., Craigie, R., and Gould, H. (1981). Regulation of the higher-order structure of chromatin by histones $\mathrm{H} 1$ and H5. J. Cell Biol. 90, 279-288.

Altaf, M., Saksouk, N., and Côté, J. (2007). Histone modifications in response to DNA damage. Mutat. Res. Mol. Mech. Mutagen. 618, 81-90.

Antoni, L., Sodha, N., Collins, I., and Garrett, M.D. (2007). CHK2 kinase: cancer susceptibility and cancer therapy - two sides of the same coin? Nat. Rev. Cancer 7, 925-936.

Audebert, M., Salles, B., and Calsou, P. (2004). Involvement of poly(ADP-ribose) polymerase1 and XRCC1/DNA ligase III in an alternative route for DNA double-strand breaks rejoining. J. Biol. Chem. 279, 55117-55126.

Ayoub, N., Jeyasekharan, A.D., Bernal, J.A., and Venkitaraman, A.R. (2008). HP1-beta mobilization promotes chromatin changes that initiate the DNA damage response. Nature 453, 682-686.

Ayoub, N., Jeyasekharan, A.D., Bernal, J.A., and Venkitaraman, A.R. (2009). Paving the way for H2AX phosphorylation: chromatin changes in the DNA damage response. Cell Cycle Georget. Tex 8, 1494-1500.

Baca, S.C., Prandi, D., Lawrence, M.S., Mosquera, J.M., Romanel, A., Drier, Y., Park, K., Kitabayashi, N., MacDonald, T.Y., Ghandi, M., et al. (2013). Punctuated Evolution of Prostate Cancer Genomes. Cell 153, 666-677.

Bakkenist, C.J., and Kastan, M.B. (2003). DNA damage activates ATM through intermolecular autophosphorylation and dimer dissociation. Nature 421, 499-506.

Banin, S., Moyal, L., Shieh, S.-Y., Taya, Y., Anderson, C.W., Chessa, L., Smorodinsky, N.I., Prives, C., Reiss, Y., Shiloh, Y., et al. (1998). Enhanced Phosphorylation of p53 by ATM in Response to DNA Damage. Science 281, 1674-1677.

Bassing, C.H., Chua, K.F., Sekiguchi, J., Suh, H., Whitlow, S.R., Fleming, J.C., Monroe, B.C., Ciccone, D.N., Yan, C., Vlasakova, K., et al. (2002). Increased ionizing radiation 
sensitivity and genomic instability in the absence of histone H2AX. Proc. Natl. Acad. Sci. U. S. A. 99, 8173-8178.

Belotserkovskaya, R., Oh, S., Bondarenko, V.A., Orphanides, G., Studitsky, V.M., and Reinberg, D. (2003). FACT facilitates transcription-dependent nucleosome alteration. Science 301, 1090-1093.

Berger, S.L. (2002). Histone modifications in transcriptional regulation. Curr. Opin. Genet. Dev. 12, 142-148.

Berger, S.L. (2007). The complex language of chromatin regulation during transcription. Nature 447, 407-412.

Bergink, S., and Jentsch, S. (2009). Principles of ubiquitin and SUMO modifications in DNA repair. Nature 458, 461-467.

Bhaumik, S.R., Smith, E., and Shilatifard, A. (2007). Covalent modifications of histones during development and disease pathogenesis. Nat. Struct. Mol. Biol. 14, 1008-1016.

Bickmore, W.A., and van Steensel, B. (2013). Genome architecture: domain organization of interphase chromosomes. Cell 152, 1270-1284.

Bird, A.W., Yu, D.Y., Pray-Grant, M.G., Qiu, Q., Harmon, K.E., Megee, P.C., Grant, P.A., Smith, M.M., and Christman, M.F. (2002). Acetylation of histone $\mathrm{H} 4$ by Esa1 is required for DNA double-strand break repair. Nature 419, 411-415.

Birnstiel, M.L., Busslinger, M., and Strub, K. (1985). Transcription termination and 3' processing: the end is in site! Cell 41, 349-359.

Bolderson, E., Tomimatsu, N., Richard, D.J., Boucher, D., Kumar, R., Pandita, T.K., Burma, S., and Khanna, K.K. (2010). Phosphorylation of Exo1 modulates homologous recombination repair of DNA double-strand breaks. Nucleic Acids Res. 38, 18211831.

Bongiorno-Borbone, L., De Cola, A., Barcaroli, D., Knight, R.A., Di Ilio, C., Melino, G., and De Laurenzi, V. (2010). FLASH degradation in response to UV-C results in histone locus bodies disruption and cell-cycle arrest. Oncogene 29, 802-810.

Bont, R.D., and Larebeke, N. van (2004). Endogenous DNA damage in humans: a review of quantitative data. Mutagenesis 19, 169-185.

Van den Bosch, M., Bree, R.T., and Lowndes, N.F. (2003). The MRN complex: coordinating and mediating the response to broken chromosomes. EMBO Rep. 4, 844-849.

Brandsma, I., and Gent, D.C. van (2012). Pathway choice in DNA double strand break repair: observations of a balancing act. Genome Integr. 3, 9.

Brush, D., Dodgson, J.B., Choi, O.R., Stevens, P.W., and Engel, J.D. (1985). Replacement variant histone genes contain intervening sequences. Mol. Cell. Biol. 5, 1307-1317. 
Bryant, H.E., Schultz, N., Thomas, H.D., Parker, K.M., Flower, D., Lopez, E., Kyle, S., Meuth, M., Curtin, N.J., and Helleday, T. (2005). Specific killing of BRCA2-deficient tumours with inhibitors of poly(ADP-ribose) polymerase. Nature 434, 913-917.

Burgess, R.J., and Zhang, Z. (2010). Roles for Gon5 in promoting nucleosome assembly and maintaining genome integrity. Cell Cycle Georget. Tex 9, 2979-2985.

Burkhardt, L., Fuchs, S., Krohn, A., Masser, S., Mader, M., Kluth, M., Bachmann, F., Huland, H., Steuber, T., Graefen, M., et al. (2013). CHD1 is a 5q21 tumor suppressor required for ERG rearrangement in prostate cancer. Cancer Res.

Cakmakci, N.G., Lerner, R.S., Wagner, E.J., Zheng, L., and Marzluff, W.F. (2008). SLIP1, a factor required for activation of histone mRNA translation by the stem-loop binding protein. Mol. Cell. Biol. 28, 1182-1194.

Camphausen, K., Brady, K.J., Burgan, W.E., Cerra, M.A., Russell, J.S., Bull, E.E.A., and Tofilon, P.J. (2004). Flavopiridol enhances human tumor cell radiosensitivity and prolongs expression of gammaH2AX foci. Mol. Cancer Ther. 3, 409-416.

Celeste, A., Petersen, S., Romanienko, P.J., Fernandez-Capetillo, O., Chen, H.T., Sedelnikova, O.A., Reina-San-Martin, B., Coppola, V., Meffre, E., Difilippantonio, M.J., et al. (2002). Genomic instability in mice lacking histone H2AX. Science 296, 922-927.

Celic, I., Masumoto, H., Griffith, W.P., Meluh, P., Cotter, R.J., Boeke, J.D., and Verreault, A. (2006). The sirtuins hst3 and Hst4p preserve genome integrity by controlling histone h3 lysine 56 deacetylation. Curr. Biol. Cb 16, 1280-1289.

Cerosaletti, K., and Concannon, P. (2004). Independent roles for nibrin and Mre11-Rad50 in the activation and function of Atm. J. Biol. Chem. 279, 38813-38819.

Chai, B., Huang, J., Cairns, B.R., and Laurent, B.C. (2005). Distinct roles for the RSC and Swi/Snf ATP-dependent chromatin remodelers in DNA double-strand break repair. Genes Dev. 19, 1656-1661.

Chan, D.W., Ye, R., Veillette, C.J., and Lees-Miller, S.P. (1999). DNA-dependent protein kinase phosphorylation sites in $\mathrm{Ku} \mathrm{70/80} \mathrm{heterodimer.} \mathrm{Biochemistry} \mathrm{(Mosc.)} \mathrm{38,}$ 1819-1828.

Chapman, J.R., Taylor, M.R.G., and Boulton, S.J. (2012). Playing the End Game: DNA DoubleStrand Break Repair Pathway Choice. Mol. Cell 47, 497-510.

Chen, C.-C., Carson, J.J., Feser, J., Tamburini, B., Zabaronick, S., Linger, J., and Tyler, J.K. (2008). Acetylated lysine 56 on histone H3 drives chromatin assembly after repair and signals for the completion of repair. Cell 134, 231-243.

Chernikova, S.B., and Brown, J.M. (2012). R-loops and genomic instability in Bre1 (RNF20/40)-deficient cells. Cell Cycle 11, 2980-2984.

Chernikova, S.B., Dorth, J.A., Razorenova, O.V., Game, J.C., and Brown, J.M. (2010). Deficiency in Bre1 impairs homologous recombination repair and cell cycle 
checkpoint response to radiation damage in mammalian cells. Radiat. Res. $174,558-$ 565.

Cheung, W.L., Turner, F.B., Krishnamoorthy, T., Wolner, B., Ahn, S.-H., Foley, M., Dorsey, J.A., Peterson, C.L., Berger, S.L., and Allis, C.D. (2005). Phosphorylation of histone H4 serine 1 during DNA damage requires casein kinase II in S. cerevisiae. Curr. Biol. Cb $15,656-660$.

Ciccia, A., and Elledge, S.J. (2010). The DNA damage response: making it safe to play with knives. Mol. Cell 40, 179-204.

Cimprich, K.A., and Cortez, D. (2008). ATR: An Essential Regulator of Genome Integrity. Nat. Rev. Mol. Cell Biol. 9, 616-627.

Collart, D., Ramsey-Ewing, A., Bortell, R., Lian, J., Stein, J., and Stein, G. (1991). Isolation and characterization of a cDNA from a human histone $\mathrm{H} 2 \mathrm{~B}$ gene which is reciprocally expressed in relation to replication-dependent $\mathrm{H} 2 \mathrm{~B}$ histone genes during HL60 cell differentiation. Biochemistry (Mosc.) 30, 1610-1617.

Collins, I., and Newlon, C.S. (1994). Meiosis-specific formation of joint DNA molecules containing sequences from homologous chromosomes. Cell 76, 65-75.

Cosgrove, M.S., and Wolberger, C. (2005). How does the histone code work? Biochem. Cell Biol. Biochim. Biol. Cell. 83, 468-476.

Courilleau, C., Chailleux, C., Jauneau, A., Grimal, F., Briois, S., Boutet-Robinet, E., Boudsocq, F., Trouche, D., and Canitrot, Y. (2012). The chromatin remodeler p400 ATPase facilitates Rad51-mediated repair of DNA double-strand breaks. J. Cell Biol. 199, 1067-1081.

Critchlow, S.E., and Jackson, S.P. (1998). DNA end-joining: from yeast to man. Trends Biochem. Sci. 23, 394-398.

Das, C., Lucia, M.S., Hansen, K.C., and Tyler, J.K. (2009). CBP/p300-mediated acetylation of histone $\mathrm{H} 3$ on lysine 56 . Nature $459,113-117$.

Dávila López, M., and Samuelsson, T. (2008). Early evolution of histone mRNA 3' end processing. Rna New York N 14, 1-10.

Dejmek, J., Iglehart, J.D., and Lazaro, J.-B. (2009). DNA-dependent protein kinase (DNA-PK)dependent cisplatin-induced loss of nucleolar facilitator of chromatin transcription (FACT) and regulation of cisplatin sensitivity by DNA-PK and FACT. Mol. Cancer Res. Mcr 7, 581-591.

DeLisle, A.J., Graves, R.A., Marzluff, W.F., and Johnson, L.F. (1983). Regulation of histone mRNA production and stability in serum-stimulated mouse 3 T6 fibroblasts. Mol. Cell. Biol. 3, 1920-1929.

Dellaire, G., Kepkay, R., and Bazett-Jones, D.P. (2009). High resolution imaging of changes in the structure and spatial organization of chromatin, gamma-H2A.X and the MRN 
complex within etoposide-induced DNA repair foci. Cell Cycle Georget. Tex 8, 37503769.

Delmas, V., Stokes, D.G., and Perry, R.P. (1993). A mammalian DNA-binding protein that contains a chromodomain and an SNF2/SWI2-like helicase domain. Proc. Natl. Acad. Sci. U. S. A. 90, 2414-2418.

Dominski, Z., and Marzluff, W.F. (2007). Formation of the 3' end of histone mRNA: getting closer to the end. Gene 396, 373-390.

Downs, J.A., Allard, S., Jobin-Robitaille, O., Javaheri, A., Auger, A., Bouchard, N., Kron, S.J., Jackson, S.P., and Côté, J. (2004). Binding of chromatin-modifying activities to phosphorylated histone H2A at DNA damage sites. Mol. Cell 16, 979-990.

Erkmann, J.A., Sànchez, R., Treichel, N., Marzluff, W.F., and Kutay, U. (2005). Nuclear export of metazoan replication-dependent histone mRNAs is dependent on RNA length and is mediated by TAP. Rna New York N 11, 45-58.

Farmer, H., McCabe, N., Lord, C.J., Tutt, A.N.J., Johnson, D.A., Richardson, T.B., Santarosa, M., Dillon, K.J., Hickson, I., Knights, C., et al. (2005). Targeting the DNA repair defect in BRCA mutant cells as a therapeutic strategy. Nature 434, 917-921.

Fernandez-Capetillo, O., Allis, C.D., and Nussenzweig, A. (2004). Phosphorylation of histone H2B at DNA double-strand breaks. J. Exp. Med. 199, 1671-1677.

Feser, J., Truong, D., Das, C., Carson, J.J., Kieft, J., Harkness, T., and Tyler, J.K. (2010). Elevated histone expression promotes life span extension. Mol. Cell 39, 724-735.

Fischle, W., Wang, Y., and Allis, C.D. (2003). Histone and chromatin cross-talk. Curr. Opin. Cell Biol. 15, 172-183.

Fleming, A.B., Kao, C.-F., Hillyer, C., Pikaart, M., and Osley, M.A. (2008). H2B ubiquitylation plays a role in nucleosome dynamics during transcription elongation. Mol. Cell 31, 57-66.

Frey, M.R., and Matera, A.G. (1995). Coiled bodies contain U7 small nuclear RNA and associate with specific DNA sequences in interphase human cells. Proc. Natl. Acad. Sci. 92, 5915-5919.

Gaillard, P.H., Martini, E.M., Kaufman, P.D., Stillman, B., Moustacchi, E., and Almouzni, G. (1996). Chromatin assembly coupled to DNA repair: a new role for chromatin assembly factor I. Cell 86, 887-896.

Gao, G., Bracken, A.P., Burkard, K., Pasini, D., Classon, M., Attwooll, C., Sagara, M., Imai, T., Helin, K., and Zhao, J. (2003). NPAT expression is regulated by E2F and is essential for cell cycle progression. Mol. Cell. Biol. 23, 2821-2833.

Gaspar-Maia, A., Alajem, A., Polesso, F., Sridharan, R., Mason, M.J., Heidersbach, A., Ramalho-Santos, J., McManus, M.T., Plath, K., Meshorer, E., et al. (2009). Chd1 regulates open chromatin and pluripotency of embryonic stem cells. Nature 460, 863-868. 
Gatti, R.A., Becker-Catania, S., Chun, H.H., Sun, X., Mitui, M., Lai, C.H., Khanlou, N., Babaei, M., Cheng, R., Clark, C., et al. (2001). The pathogenesis of ataxia-telangiectasia. Learning from a Rosetta Stone. Clin. Rev. Allergy Immunol. 20, 87-108.

Ghule, P.N., Dominski, Z., Yang, X.-C., Marzluff, W.F., Becker, K.A., Harper, J.W., Lian, J.B., Stein, J.L., van Wijnen, A.J., and Stein, G.S. (2008). Staged assembly of histone gene expression machinery at subnuclear foci in the abbreviated cell cycle of human embryonic stem cells. Proc. Natl. Acad. Sci. U. S. A. 105, 16964-16969.

Giannattasio, M., Lazzaro, F., Plevani, P., and Muzi-Falconi, M. (2005). The DNA Damage Checkpoint Response Requires Histone H2B Ubiquitination by Rad6-Bre1 and H3 Methylation by Dot1. J. Biol. Chem. 280, 9879-9886.

Gkikopoulos, T., Schofield, P., Singh, V., Pinskaya, M., Mellor, J., Smolle, M., Workman, J.L., Barton, G.J., and Owen-Hughes, T. (2011). A Role for Snf2-Related NucleosomeSpacing Enzymes in Genome-Wide Nucleosome Organization. Science 333, 17581760.

Goldberg, M., Stucki, M., Falck, J., D’Amours, D., Rahman, D., Pappin, D., Bartek, J., and Jackson, S.P. (2003). MDC1 is required for the intra-S-phase DNA damage checkpoint. Nature 421, 952-956.

Gomes, N.P., Bjerke, G., Llorente, B., Szostek, S.A., Emerson, B.M., and Espinosa, J.M. (2006). Gene-specific requirement for P-TEFb activity and RNA polymerase II phosphorylation within the p53 transcriptional program. Genes Dev. 20, 601-612.

Goodarzi, A.A., Yu, Y., Riballo, E., Douglas, P., Walker, S.A., Ye, R., Härer, C., Marchetti, C., Morrice, N., Jeggo, P.A., et al. (2006). DNA-PK autophosphorylation facilitates Artemis endonuclease activity. Embo J. 25, 3880-3889.

Goodarzi, A.A., Jeggo, P., and Lobrich, M. (2010). The influence of heterochromatin on DNA double strand break repair: Getting the strong, silent type to relax. Dna Repair 9, 1273-1282.

Gottlieb, T.M., and Jackson, S.P. (1993). The DNA-dependent protein kinase: requirement for DNA ends and association with Ku antigen. Cell 72, 131-142.

Grasso, C.S., Wu, Y.-M., Robinson, D.R., Cao, X., Dhanasekaran, S.M., Khan, A.P., Quist, M.J., Jing, X., Lonigro, R.J., Brenner, J.C., et al. (2012). The mutational landscape of lethal castration-resistant prostate cancer. Nature 487, 239-243.

Grewal, S.I.S., and Elgin, S.C.R. (2007). Transcription and RNA interference in the formation of heterochromatin. Nature 447, 399-406.

Hacques, M.F., Muller, S., De Murcia, G., Van Regenmortel, M.H., and Marion, C. (1990). Use of an immobilized enzyme and specific antibodies to analyse the accessibility and role of histone tails in chromatin structure. Biochem. Biophys. Res. Commun. 168, 637-643.

Harper, J.W., and Elledge, S.J. (2007). The DNA Damage Response: Ten Years After. Mol. Cell $28,739-745$. 
Harvey, A.C., Jackson, S.P., and Downs, J.A. (2005). Saccharomyces cerevisiae histone H2A Ser122 facilitates DNA repair. Genetics 170, 543-553.

Hauk, G., McKnight, J.N., Nodelman, I.M., and Bowman, G.D. (2010). The chromodomains of the Chd1 chromatin remodeler regulate DNA access to the ATPase motor. Mol. Cell 39, 711-723.

Helleday, T., Petermann, E., Lundin, C., Hodgson, B., and Sharma, R.A. (2008). DNA repair pathways as targets for cancer therapy. Nat. Rev. Cancer 8, 193-204.

Hennig, B.P., Bendrin, K., Zhou, Y., and Fischer, T. (2012). Chd1 chromatin remodelers maintain nucleosome organization and repress cryptic transcription. EMBO Rep. 13, 997-1003.

Henry, K.W., Wyce, A., Lo, W.-S., Duggan, L.J., Emre, N.C.T., Kao, C.-F., Pillus, L., Shilatifard, A., Osley, M.A., and Berger, S.L. (2003). Transcriptional activation via sequential histone $\mathrm{H} 2 \mathrm{~B}$ ubiquitylation and deubiquitylation, mediated by SAGA-associated Ubp8. Genes Dev. 17, 2648-2663.

Heo, K., Kim, H., Choi, S.H., Choi, J., Kim, K., Gu, J., Lieber, M.R., Yang, A.S., and An, W. (2008). FACT-mediated exchange of histone variant $H 2 A X$ regulated by phosphorylation of H2AX and ADP-ribosylation of Spt16. Mol. Cell 30, 86-97.

Heyer, W.-D., Ehmsen, K.T., and Liu, J. (2010). Regulation of homologous recombination in eukaryotes. Annu. Rev. Genet. 44, 113-139.

Hondele, M., Stuwe, T., Hassler, M., Halbach, F., Bowman, A., Zhang, E.T., Nijmeijer, B., Kotthoff, C., Rybin, V., Amlacher, S., et al. (2013). Structural basis of histone H2A-H2B recognition by the essential chaperone FACT. Nature advance online publication.

Huang, Y., and Steitz, J.A. (2001). Splicing factors SRp20 and 9 G8 promote the nucleocytoplasmic export of mRNA. Mol. Cell 7, 899-905.

Huang, S., Gulzar, Z.G., Salari, K., Lapointe, J., Brooks, J.D., and Pollack, J.R. (2012). Recurrent deletion of CHD1 in prostate cancer with relevance to cell invasiveness. Oncogene 31, 4164-4170.

Huen, M.S.Y., Sy, S.M.H., and Chen, J. (2010). BRCA1 and its toolbox for the maintenance of genome integrity. Nat. Rev. Mol. Cell Biol. 11, 138-148.

Hyland, E.M., Cosgrove, M.S., Molina, H., Wang, D., Pandey, A., Cottee, R.J., and Boeke, J.D. (2005). Insights into the Role of Histone H3 and Histone H4 Core Modifiable Residues in Saccharomyces cerevisiae. Mol. Cell. Biol. 25, 10060-10070.

Iacovoni, J.S., Caron, P., Lassadi, I., Nicolas, E., Massip, L., Trouche, D., and Legube, G. (2010). High-resolution profiling of ?H2AX around DNA double strand breaks in the mammalian genome. Embo J. 29, 1446-1457.

Ikura, T., Ogryzko, V.V., Grigoriev, M., Groisman, R., Wang, J., Horikoshi, M., Scully, R., Qin, J., and Nakatani, Y. (2000). Involvement of the TIP60 histone acetylase complex in DNA repair and apoptosis. Cell 102, 463-473. 
Jackson, S.P., and Bartek, J. (2009). The DNA-damage response in human biology and disease. Nature 461, 1071-1078.

Jackson, S.P., and Durocher, D. (2013). Regulation of DNA Damage Responses by Ubiquitin and SUMO. Mol. Cell 49, 795-807.

Jenuwein, T., and Allis, C.D. (2001). Translating the histone code. Science 293, 1074-1080.

Jha, S., Shibata, E., and Dutta, A. (2008). Human Rvb1/Tip49 is required for the histone acetyltransferase activity of Tip60/NuA4 and for the downregulation of phosphorylation on H2AX after DNA damage. Mol. Cell. Biol. 28, 2690-2700.

Jiricny, J. (2006). The multifaceted mismatch-repair system. Nat. Rev. Mol. Cell Biol. 7, 335346.

Jørgensen, S., Elvers, I., Trelle, M.B., Menzel, T., Eskildsen, M., Jensen, O.N., Helleday, T., Helin, K., and Sørensen, C.S. (2007). The histone methyltransferase SET8 is required for S-phase progression. J. Cell Biol. 179, 1337-1345.

Jung, C., Motwani, M., Kortmansky, J., Sirotnak, F.M., She, Y., Gonen, M., HaimovitzFriedman, A., and Schwartz, G.K. (2003). The cyclin-dependent kinase inhibitor flavopiridol potentiates gamma-irradiation-induced apoptosis in colon and gastric cancer cells. Clin. Cancer Res. Off. J. Am. Assoc. Cancer Res. 9, 6052-6061.

Kari, V., Shchebet, A., Neumann, H., and Johnsen, S.A. (2011). The H2B ubiquitin ligase RNF40 cooperates with SUPT16H to induce dynamic changes in chromatin structure during DNA double-strand break repair. Cell Cycle Georget. Tex 10, 3495-3504.

Karpiuk, O., Najafova, Z., Kramer, F., Hennion, M., Galonska, C., König, A., Snaidero, N., Vogel, T., Shchebet, A., Begus-Nahrmann, Y., et al. (2012). The histone H2B monoubiquitination regulatory pathway is required for differentiation of multipotent stem cells. Mol. Cell 46, 705-713.

Kelley, D.E., Stokes, D.G., and Perry, R.P. (1999). CHD1 interacts with SSRP1 and depends on both its chromodomain and its ATPase/helicase-like domain for proper association with chromatin. Chromosoma 108, 10-25.

Kim, J., Guermah, M., McGinty, R.K., Lee, J.-S., Tang, Z., Milne, T.A., Shilatifard, A., Muir, T.W., and Roeder, R.G. (2009). RAD6-Mediated transcription-coupled H2B ubiquitylation directly stimulates H3K4 methylation in human cells. Cell 137, 459471.

Kireeva, M.L., Walter, W., Tchernajenko, V., Bondarenko, V., Kashlev, M., and Studitsky, V.M. (2002). Nucleosome remodeling induced by RNA polymerase II: loss of the $\mathrm{H} 2 \mathrm{~A} / \mathrm{H} 2 \mathrm{~B}$ dimer during transcription. Mol. Cell 9, 541-552.

Kirsh, A.L., Groudine, M., and Challoner, P.B. (1989). Polyadenylation and U7 snRNPmediated cleavage: alternative modes of RNA 3' processing in two avian histone H1 genes. Genes Dev. 3, 2172-2179. 
Kolev, N.G., and Steitz, J.A. (2005). Symplekin and multiple other polyadenylation factors participate in 3'-end maturation of histone mRNAs. Genes Dev. 19, 2583-2592.

Kolev, N.G., Yario, T.A., Benson, E., and Steitz, J.A. (2008). Conserved motifs in both CPSF73 and CPSF100 are required to assemble the active endonuclease for histone mRNA $3^{\prime}$ end maturation. EMBO Rep. 9, 1013-1018.

Kondo, T., Wakayama, T., Naiki, T., Matsumoto, K., and Sugimoto, K. (2001). Recruitment of Mec1 and Ddc1 checkpoint proteins to double-strand breaks through distinct mechanisms. Science 294, 867-870.

Kornberg, R.D. (1974). Chromatin Structure: A Repeating Unit of Histones and DNA. Science $184,868-871$.

Kornberg, R.D., and Lorch, Y. (1999). Twenty-Five Years of the Nucleosome, Fundamental Particle of the Eukaryote Chromosome. Cell 98, 285-294.

Kouzarides, T. (2007). Chromatin modifications and their function. Cell 128, 693-705.

Kozlov, S.V., Graham, M.E., Jakob, B., Tobias, F., Kijas, A.W., Tanuji, M., Chen, P., Robinson, P.J., Taucher-Scholz, G., Suzuki, K., et al. (2011). Autophosphorylation and ATM Activation ADDITIONAL SITES ADD TO THE COMPLEXITY. J. Biol. Chem. 286, 91079119.

Lans, H., Marteijn, J.A., and Vermeulen, W. (2012). ATP-dependent chromatin remodeling in the DNA-damage response. Epigenetics Chromatin 5, 4.

Laribee, R.N., Fuchs, S.M., and Strahl, B.D. (2007). H2B ubiquitylation in transcriptional control: a FACT-finding mission. Genes Dev. 21, 737-743.

Larsen, D.H., Poinsignon, C., Gudjonsson, T., Dinant, C., Payne, M.R., Hari, F.J., Danielsen, J.M.R., Menard, P., Sand, J.C., Stucki, M., et al. (2010). The chromatin-remodeling factor CHD4 coordinates signaling and repair after DNA damage. J. Cell Biol. 190, 731-740.

Lavin, M.F. (2008). Ataxia-telangiectasia: from a rare disorder to a paradigm for cell signalling and cancer. Nat. Rev. Mol. Cell Biol. 9, 759-769.

Leber, R., Wise, T.W., Mizuta, R., and Meek, K. (1998). The XRCC4 gene product is a target for and interacts with the DNA-dependent protein kinase. J. Biol. Chem. 273, 17941801.

Lee, H.-S., Park, J.-H., Kim, S.-J., Kwon, S.-J., and Kwon, J. (2010). A cooperative activation loop among SWI/SNF, ?-H2AX and H3 acetylation for DNA double-strand break repair. Embo J. 29, 1434-1445.

Lee, J.-H., Mand, M.R., Deshpande, R., Kinoshita, E., Yang, S.-H., Wyman, C., and Paull, T.T. (2013). ATM kinase activity is regulated by ATP-driven conformational changes in the MRN complex. J. Biol. Chem. 
Lee, J.-S., Garrett, A.S., Yen, K., Takahashi, Y.-H., Hu, D., Jackson, J., Seidel, C., Pugh, B.F., and Shilatifard, A. (2012). Codependency of H2B monoubiquitination and nucleosome reassembly on Chd1. Genes Dev. 26, 914-919.

Lemaître, C., Fischer, B., Kalousi, A., Hoffbeck, A.-S., Guirouilh-Barbat, J., Shahar, O.D., Genet, D., Goldberg, M., Betrand, P., Lopez, B., et al. (2012). The nucleoporin 153, a novel factor in double-strand break repair and DNA damage response. Oncogene 31 , 4803-4809.

Li, Q., Zhou, H., Wurtele, H., Davies, B., Horazdovsky, B., Verreault, A., and Zhang, Z. (2008). Acetylation of histone $\mathrm{H} 3$ lysine 56 regulates replication-coupled nucleosome assembly. Cell 134, 244-255.

Lieber, M.R. (2010). The mechanism of double-strand DNA break repair by the nonhomologous DNA end-joining pathway. Annu. Rev. Biochem. 79, 181-211.

Lim, D.S., Kim, S.T., Xu, B., Maser, R.S., Lin, J., Petrini, J.H., and Kastan, M.B. (2000). ATM phosphorylates p95/nbs1 in an S-phase checkpoint pathway. Nature 404, 613-617.

Limbo, O., Chahwan, C., Yamada, Y., de Bruin, R.A.M., Wittenberg, C., and Russell, P. (2007). Ctp1 is a cell-cycle-regulated protein that functions with Mre11 complex to control double-strand break repair by homologous recombination. Mol. Cell 28, 134-146.

Lindahl, T., and Barnes, D.E. (2000). Repair of Endogenous DNA Damage. Cold Spring Harb. Symp. Quant. Biol. 65, 127-134.

Liu, J.-L., Murphy, C., Buszczak, M., Clatterbuck, S., Goodman, R., and Gall, J.G. (2006). The Drosophila melanogaster Cajal body. J. Cell Biol. 172, 875-884.

Longhese, M.P., Foiani, M., Muzi-Falconi, M., Lucchini, G., and Plevani, P. (1998). DNA damage checkpoint in budding yeast. Embo J. 17, 5525-5528.

Lord, C.J., and Ashworth, A. (2012). The DNA damage response and cancer therapy. Nature 481, 287-294.

Luger, K., Mäder, A.W., Richmond, R.K., Sargent, D.F., and Richmond, T.J. (1997). Crystal structure of the nucleosome core particle at 2.8 A resolution. Nature $389,251-260$.

Luk, E., Vu, N.-D., Patteson, K., Mizuguchi, G., Wu, W.-H., Ranjan, A., Backus, J., Sen, S., Lewis, M., Bai, Y., et al. (2007). Chz1, a nuclear chaperone for histone H2AZ. Mol. Cell 25, 357-368.

Lukas, J., Lukas, C., and Bartek, J. (2011). More than just a focus: The chromatin response to DNA damage and its role in genome integrity maintenance. Nat. Cell Biol. 13, 11611169.

Lusser, A., Urwin, D.L., and Kadonaga, J.T. (2005). Distinct activities of CHD1 and ACF in ATPdependent chromatin assembly. Nat. Struct. Mol. Biol. 12, 160-166.

Ma, T., Van Tine, B.A., Wei, Y., Garrett, M.D., Nelson, D., Adams, P.D., Wang, J., Qin, J., Chow, L.T., and Harper, J.W. (2000). Cell cycle-regulated phosphorylation of 
p220(NPAT) by cyclin E/Cdk2 in Cajal bodies promotes histone gene transcription. Genes Dev. 14, 2298-2313.

Ma, Y., Lu, H., Schwarz, K., and Lieber, M.R. (2005). Repair of double-strand DNA breaks by the human nonhomologous DNA end joining pathway: the iterative processing model. Cell Cycle Georget. Tex 4, 1193-1200.

Maas, N.L., Miller, K.M., DeFazio, L.G., and Toczyski, D.P. (2006). Cell cycle and checkpoint regulation of histone H3 K56 acetylation by Hst3 and Hst4. Mol. Cell 23, 109-119.

Mahaney, B.L., Meek, K., and Lees-Miller, S.P. (2009). Repair of ionizing radiation-induced DNA double-strand breaks by non-homologous end-joining. Biochem. J. 417, 639650.

Maloisel, L., Fabre, F., and Gangloff, S. (2008). DNA Polymerase ? Is Preferentially Recruited during Homologous Recombination To Promote Heteroduplex DNA Extension. Mol. Cell. Biol. 28, 1373-1382.

Mangone, M., Manoharan, A.P., Thierry-Mieg, D., Thierry-Mieg, J., Han, T., Mackowiak, S.D., Mis, E., Zegar, C., Gutwein, M.R., Khivansara, V., et al. (2010). The landscape of C. elegans 3'UTRs. Science 329, 432-435.

Mannironi, C., Bonner, W.M., and Hatch, C.L. (1989). H2A.X. a histone isoprotein with a conserved C-terminal sequence, is encoded by a novel mRNA with both DNA replication type and polyA 3' processing signals. Nucleic Acids Res. 17, 9113-9126.

Mao, Z., Bozzella, M., Seluanov, A., and Gorbunova, V. (2008). DNA repair by nonhomologous end joining and homologous recombination during cell cycle in human cells. Cell Cycle Georget. Tex 7, 2902-2906.

Marfella, C.G.A., and Imbalzano, A.N. (2007). The Chd family of chromatin remodelers. Mutat. Res. Mol. Mech. Mutagen. 618, 30-40.

Martinez, I., Wang, J., Hobson, K.F., Ferris, R.L., and Khan, S.A. (2007). Identification of differentially expressed genes in HPV-positive and HPV-negative oropharyngeal squamous cell carcinomas. Eur. J. Cancer Oxf. Engl. 1990 43, 415-432.

Marzluff, W.F. (1992). Histone 3' ends: essential and regulatory functions. Gene Expr. 2, 9397.

Marzluff, W.F., Gongidi, P., Woods, K.R., Jin, J., and Maltais, L.J. (2002). The human and mouse replication-dependent histone genes. Genomics 80, 487-498.

Marzluff, W.F., Wagner, E.J., and Duronio, R.J. (2008). Metabolism and regulation of canonical histone mRNAs: life without a poly(A) tail. Nat. Rev. Genet. 9, 843-854.

Mašek, T., Valášek, L., and Pospíšek, M. (2011). Polysome analysis and RNA purification from sucrose gradients. Methods Mol. Biol. Clifton Nj 703, 293-309.

Massip, L., Caron, P., lacovoni, J.S., Trouche, D., and Legube, G. (2010). Deciphering the chromatin landscape induced around DNA double strand breaks. Cell Cycle Georget. Tex 9, 2963-2972. 
Matera, A.G. (2006). Drosophila Cajal bodies: accessories not included. J. Cell Biol. 172, 791793.

Matsuoka, S., Ballif, B.A., Smogorzewska, A., McDonald, E.R., Hurov, K.E., Luo, J., Bakalarski, C.E., Zhao, Z., Solimini, N., Lerenthal, Y., et al. (2007). ATM and ATR Substrate Analysis Reveals Extensive Protein Networks Responsive to DNA Damage. Science 316, 1160-1166.

Mazumdar, A., Wang, R.A., Mishra, S.K., Adam, L., Bagheri-Yarmand, R., Mandal, M., Vadlamudi, R.K., and Kumar, R. (2001). Transcriptional repression of oestrogen receptor by metastasis-associated protein 1 corepressor. Nat. Cell Biol. 3, 30-37.

McCord, R.A., Michishita, E., Hong, T., Berber, E., Boxer, L.D., Kusumoto, R., Guan, S., Shi, X., Gozani, O., Burlingame, A.L., et al. (2009). SIRT6 stabilizes DNA-dependent protein kinase at chromatin for DNA double-strand break repair. Aging (Albany NY) 1, 109121.

McDaniel, I.E., Lee, J.M., Berger, M.S., Hanagami, C.K., and Armstrong, J.A. (2008). Investigations of CHD1 Function in Transcription and Development of Drosophila melanogaster. Genetics 178, 583-587.

McKinnon, P.J. (2012). ATM and the molecular pathogenesis of ataxia telangiectasia. Annu. Rev. Pathol. 7, 303-321.

Meek, K., Dang, V., and Lees-Miller, S.P. (2008). DNA-PK: the means to justify the ends? Adv. Immunol. 99, 33-58.

Mello, M.L.S. (1983). Cytochemical properties of euchromatin and heterochromatin. Histochem. J. 15, 739-751.

Mello, J.A., Silljé, H.H.W., Roche, D.M.J., Kirschner, D.B., Nigg, E.A., and Almouzni, G. (2002). Human Asf1 and CAF-1 interact and synergize in a repair-coupled nucleosome assembly pathway. EMBO Rep. 3, 329-334.

Minsky, N., Shema, E., Field, Y., Schuster, M., Segal, E., and Oren, M. (2008). Monoubiquitinated $\mathrm{H} 2 \mathrm{~B}$ is associated with the transcribed region of highly expressed genes in human cells. Nat. Cell Biol. 10, 483-488.

Misteli, T., and Soutoglou, E. (2009). The emerging role of nuclear architecture in DNA repair and genome maintenance. Nat. Rev. Mol. Cell Biol. 10, 243-254.

Moldovan, G.-L., and D'Andrea, A.D. (2009). How the fanconi anemia pathway guards the genome. Annu. Rev. Genet. 43, 223-249.

Morrison, A.J., Highland, J., Krogan, N.J., Arbel-Eden, A., Greenblatt, J.F., Haber, J.E., and Shen, X. (2004). INO80 and gamma-H2AX interaction links ATP-dependent chromatin remodeling to DNA damage repair. Cell 119, 767-775.

Mowry, K.L., and Steitz, J.A. (1987). Identification of the human U7 snRNP as one of several factors involved in the $3^{\prime}$ end maturation of histone premessenger RNA's. Science 238, 1682-1687. 
Moyal, L., Lerenthal, Y., Gana-Weisz, M., Mass, G., So, S., Wang, S.-Y., Eppink, B., Chung, Y.M., Shalev, G., Shema, E., et al. (2011). Requirement of ATM-dependent monoubiquitylation of histone H2B for timely repair of DNA double-strand breaks. Mol. Cell 41, 529-542.

Mullen, T.E., and Marzluff, W.F. (2008). Degradation of histone mRNA requires oligouridylation followed by decapping and simultaneous degradation of the mRNA both 5' to 3' and 3' to 5'. Genes Dev. 22, 50-65.

Mund, A., Schubert, T., Staege, H., Kinkley, S., Reumann, K., Kriegs, M., Fritsch, L., Battisti, V., Ait-Si-Ali, S., Hoffbeck, A.-S., et al. (2012). SPOC1 modulates DNA repair by regulating key determinants of chromatin compaction and DNA damage response. Nucleic Acids Res. 40, 11363-11379.

Murr, R., Vaissière, T., Sawan, C., Shukla, V., and Herceg, Z. (2007). Orchestration of chromatin-based processes: mind the TRRAP. Oncogene 26, 5358-5372.

Nair, D.M., Ge, Z., Mersfelder, E.L., and Parthun, M.R. (2011). Genetic interactions between POB3 and the acetylation of newly synthesized histones. Curr. Genet. 57, 271-286.

Nakamura, A.J., Rao, V.A., Pommier, Y., and Bonner, W.M. (2010). The complexity of phosphorylated $\mathrm{H} 2 \mathrm{AX}$ foci formation and DNA repair assembly at DNA double-strand breaks. Cell Cycle Georget. Tex 9, 389-397.

Nakamura, K., Kato, A., Kobayashi, J., Yanagihara, H., Sakamoto, S., Oliveira, D.V.N.P., Shimada, M., Tauchi, H., Suzuki, H., Tashiro, S., et al. (2011). Regulation of homologous recombination by RNF20-dependent H2B ubiquitination. Mol. Cell 41, 515-528.

Narita, T., Yung, T.M.C., Yamamoto, J., Tsuboi, Y., Tanabe, H., Tanaka, K., Yamaguchi, Y., and Handa, H. (2007). NELF interacts with CBC and participates in $3^{\prime}$ end processing of replication-dependent histone mRNAs. Mol. Cell 26, 349-365.

Narlikar, G.J., Fan, H.-Y., and Kingston, R.E. (2002). Cooperation between Complexes that Regulate Chromatin Structure and Transcription. Cell 108, 475-487.

Neumann, H., Hancock, S.M., Buning, R., Routh, A., Chapman, L., Somers, J., Owen-Hughes, T., van Noort, J., Rhodes, D., and Chin, J.W. (2009). A method for genetically installing site-specific acetylation in recombinant histones defines the effects of $\mathrm{H} 3$ K56 acetylation. Mol. Cell 36, 153-163.

Ng, H.H., Xu, R.-M., Zhang, Y., and Struhl, K. (2002). Ubiquitination of Histone H2B by Rad6 Is Required for Efficient Dot1-mediated Methylation of Histone H3 Lysine 79. J. Biol. Chem. 277, 34655-34657.

Nicolson, G.L., Nawa, A., Toh, Y., Taniguchi, S., Nishimori, K., and Moustafa, A. (2003). Tumor metastasis-associated human MTA1 gene and its MTA1 protein product: role in epithelial cancer cell invasion, proliferation and nuclear regulation. Clin. Exp. Metastasis 20, 19-24.

Oberdoerffer, P. (2010). An age of fewer histones. Nat. Cell Biol. 12, 1029-1031. 
Oda, H., Okamoto, I., Murphy, N., Chu, J., Price, S.M., Shen, M.M., Torres-Padilla, M.E., Heard, E., and Reinberg, D. (2009). Monomethylation of Histone H4-Lysine 20 Is Involved in Chromosome Structure and Stability and Is Essential for Mouse Development. Mol. Cell. Biol. 29, 2278-2295.

Orphanides, G., LeRoy, G., Chang, C.H., Luse, D.S., and Reinberg, D. (1998). FACT, a factor that facilitates transcript elongation through nucleosomes. Cell 92, 105-116.

Osley, M.A. (1991). The regulation of histone synthesis in the cell cycle. Annu. Rev. Biochem. $60,827-861$.

Ouchi, M., and Ouchi, T. (2010). Regulation of ATM/DNA-PKcs Phosphorylation by BRCA1Associated BAAT1. Genes Cancer 1, 1211-1214.

Pandey, N.B., Chodchoy, N., Liu, T.J., and Marzluff, W.F. (1990). Introns in histone genes alter the distribution of 3' ends. Nucleic Acids Res. 18, 3161-3170.

Park, J.-H., Park, E.-J., Lee, H.-S., Kim, S.J., Hur, S.-K., Imbalzano, A.N., and Kwon, J. (2006). Mammalian SWI/SNF complexes facilitate DNA double-strand break repair by promoting gamma-H2AX induction. Embo J. 25, 3986-3997.

Parrilla-Castellar, E.R., Arlander, S.J.H., and Karnitz, L. (2004). Dial 9-1-1 for DNA damage: the Rad9-Hus1-Rad1 (9-1-1) clamp complex. Dna Repair 3, 1009-1014.

Pavri, R., Zhu, B., Li, G., Trojer, P., Mandal, S., Shilatifard, A., and Reinberg, D. (2006). Histone $\mathrm{H} 2 \mathrm{~B}$ monoubiquitination functions cooperatively with FACT to regulate elongation by RNA polymerase II. Cell 125, 703-717.

Pervan, M., Pajonk, F., Sun, J.R., Withers, H.R., and McBride, W.H. (2001). Molecular pathways that modify tumor radiation response. Am. J. Clin. Oncol. 24, 481-485.

Pirngruber, J., and Johnsen, S.A. (2010). Induced G1 cell-cycle arrest controls replicationdependent histone mRNA $3^{\prime}$ end processing through p21, NPAT and CDK9. Oncogene $29,2853-2863$.

Pirngruber, J., Shchebet, A., Schreiber, L., Shema, E., Minsky, N., Chapman, R.D., Eick, D., Aylon, Y., Oren, M., and Johnsen, S.A. (2009a). CDK9 directs H2B monoubiquitination and controls replication-dependent histone mRNA 3 '-end processing. EMBO Rep. 10, 894-900.

Pirngruber, J., Shchebet, A., and Johnsen, S.A. (2009b). Insights into the function of the human P-TEFb component CDK9 in the regulation of chromatin modifications and COtranscriptional mRNA processing. Cell Cycle Georget. Tex 8, 3636-3642.

Pointner, J., Persson, J., Prasad, P., Norman-Axelsson, U., Strålfors, A., Khorosjutina, O., Krietenstein, N., Svensson, J.P., Ekwall, K., and Korber, P. (2012). CHD1 remodelers regulate nucleosome spacing in vitro and align nucleosomal arrays over gene coding regions in S. pombe. Embo J. 31, 4388-4403. 
Pray-Grant, M.G., Daniel, J.A., Schieltz, D., Yates, J.R., 3rd, and Grant, P.A. (2005). Chd1 chromodomain links histone H3 methylation with SAGA- and SLIK-dependent acetylation. Nature 433, 434-438.

Prenzel, T., Begus-Nahrmann, Y., Kramer, F., Hennion, M., Hsu, C., Gorsler, T., Hintermair, C., Eick, D., Kremmer, E., Simons, M., et al. (2011). Estrogen-dependent gene transcription in human breast cancer cells relies upon proteasome-dependent monoubiquitination of histone H2B. Cancer Res. 71, 5739-5753.

Price, B.D., and D'Andrea, A.D. (2013). Chromatin remodeling at DNA double-strand breaks. Cell 152, 1344-1354.

Ransom, M., Dennehey, B.K., and Tyler, J.K. (2010). Chaperoning Histones during DNA Replication and Repair. Cell 140, 183-195.

Redon, C.E., Nakamura, A.J., Martin, O.A., Parekh, P.R., Weyemi, U.S., and Bonner, W.M. (2011). Recent developments in the use of $y-\mathrm{H} 2 \mathrm{AX}$ as a quantitative DNA doublestrand break biomarker. Aging (Albany NY) 3, 168-174.

Rogakou, E.P., Pilch, D.R., Orr, A.H., Ivanova, V.S., and Bonner, W.M. (1998a). DNA doublestranded breaks induce histone H2AX phosphorylation on serine 139. J. Biol. Chem. $273,5858-5868$.

Rogakou, E.P., Pilch, D.R., Orr, A.H., Ivanova, V.S., and Bonner, W.M. (1998b). DNA doublestranded breaks induce histone H2AX phosphorylation on serine 139. J. Biol. Chem. $273,5858-5868$.

Rossetto, D., Truman, A.W., Kron, S.J., and Côté, J. (2010). Epigenetic modifications in double-strand break DNA damage signaling and repair. Clin. Cancer Res. Off. J. Am. Assoc. Cancer Res. 16, 4543-4552.

Russo, S.M., Tepper, J.E., Baldwin, A.S., Jr, Liu, R., Adams, J., Elliott, P., and Cusack, J.C., Jr (2001). Enhancement of radiosensitivity by proteasome inhibition: implications for a role of NF-kappaB. Int. J. Radiat. Oncol. Biol. Phys. 50, 183-193.

Sancar, A., Lindsey-Boltz, L.A., Unsal-Kaçmaz, K., and Linn, S. (2004). Molecular mechanisms of mammalian DNA repair and the DNA damage checkpoints. Annu. Rev. Biochem. 73, 39-85.

Sand-Dejmek, J., Adelmant, G., Sobhian, B., Calkins, A.S., Marto, J., Iglehart, D.J., and Lazaro, J.-B. (2011). Concordant and opposite roles of DNA-PK and the "facilitator of chromatin transcription" (FACT) in DNA repair, apoptosis and necrosis after cisplatin. Mol. Cancer 10, 74.

Sanders, S.L., Portoso, M., Mata, J., Bähler, J., Allshire, R.C., and Kouzarides, T. (2004). Methylation of Histone H4 Lysine 20 Controls Recruitment of Crb2 to Sites of DNA Damage. Cell 119, 603-614.

Sartori, A.A., Lukas, C., Coates, J., Mistrik, M., Fu, S., Bartek, J., Baer, R., Lukas, J., and Jackson, S.P. (2007). Human CtIP promotes DNA end resection. Nature 450, 509-514. 
Savitsky, K., Bar-Shira, A., Gilad, S., Rotman, G., Ziv, Y., Vanagaite, L., Tagle, D.A., Smith, S., Uziel, T., Sfez, S., et al. (1995). A single ataxia telangiectasia gene with a product similar to PI-3 kinase. Science 268, 1749-1753.

Schmidt, D.R., and Schreiber, S.L. (1999). Molecular Association between ATR and Two Components of the Nucleosome Remodeling and Deacetylating Complex, HDAC2 and CHD4t. Biochemistry (Mosc.) 38, 14711-14717.

Schreiber, V., Dantzer, F., Ame, J.-C., and de Murcia, G. (2006). Poly(ADP-ribose): novel functions for an old molecule. Nat. Rev. Mol. Cell Biol. 7, 517-528.

Schultz, L.B., Chehab, N.H., Malikzay, A., and Halazonetis, T.D. (2000). p53 binding protein 1 (53BP1) is an early participant in the cellular response to DNA double-strand breaks. J. Cell Biol. 151, 1381-1390.

Scrima, A., Fischer, E.S., Lingaraju, G.M., Böhm, K., Cavadini, S., and Thomä, N.H. (2011). Detecting UV-lesions in the genome: The modular CRL4 ubiquitin ligase does it best! Febs Lett. 585, 2818-2825.

Shema, E., Tirosh, I., Aylon, Y., Huang, J., Ye, C., Moskovits, N., Raver-Shapira, N., Minsky, N., Pirngruber, J., Tarcic, G., et al. (2008). The histone H2B-specific ubiquitin ligase RNF20/hBRE1 acts as a putative tumor suppressor through selective regulation of gene expression. Genes Dev. 22, 2664-2676.

Shepard, P.J., Choi, E.-A., Lu, J., Flanagan, L.A., Hertel, K.J., and Shi, Y. (2011). Complex and dynamic landscape of RNA polyadenylation revealed by PAS-Seq. Rna New York N 17, 761-772.

Shiloh, Y. (2003). ATM and related protein kinases: safeguarding genome integrity. Nat. Rev. Cancer 3, 155-168.

Shogren-Knaak, M., Ishii, H., Sun, J.-M., Pazin, M.J., Davie, J.R., and Peterson, C.L. (2006). Histone H4-K16 acetylation controls chromatin structure and protein interactions. Science 311, 844-847.

Simic, R., Lindstrom, D.L., Tran, H.G., Roinick, K.L., Costa, P.J., Johnson, A.D., Hartzog, G.A., and Arndt, K.M. (2003). Chromatin remodeling protein Chd1 interacts with transcription elongation factors and localizes to transcribed genes. Embo J. 22, 1846-1856.

Simon, J.M., Giresi, P.G., Davis, I.J., and Lieb, J.D. (2012). Using formaldehyde-assisted isolation of regulatory elements (FAIRE) to isolate active regulatory DNA. Nat. Protoc. 7, 256-267.

Simonsen, J.L., Rosada, C., Serakinci, N., Justesen, J., Stenderup, K., Rattan, S.I.S., Jensen, T.G., and Kassem, M. (2002). Telomerase expression extends the proliferative lifespan and maintains the osteogenic potential of human bone marrow stromal cells. Nat. Biotechnol. 20, 592-596.

Sims, R.J., 3rd, Chen, C.-F., Santos-Rosa, H., Kouzarides, T., Patel, S.S., and Reinberg, D. (2005). Human but not yeast CHD1 binds directly and selectively to histone H3 
methylated at lysine 4 via its tandem chromodomains. J. Biol. Chem. 280, 4178941792.

Smerdon, M.J. (1991). DNA repair and the role of chromatin structure. Curr. Opin. Cell Biol. 3, 422-428.

Smolle, M., Venkatesh, S., Gogol, M.M., Li, H., Zhang, Y., Florens, L., Washburn, M.P., and Workman, J.L. (2012). Chromatin remodelers Isw1 and Chd1 maintain chromatin structure during transcription by preventing histone exchange. Nat. Struct. Mol. Biol. $19,884-892$.

Srivastava, M., Nambiar, M., Sharma, S., Karki, S.S., Goldsmith, G., Hegde, M., Kumar, S., Pandey, M., Singh, R.K., Ray, P., et al. (2012). An inhibitor of nonhomologous endjoining abrogates double-strand break repair and impedes cancer progression. Cell $151,1474-1487$.

Stiff, T., O'Driscoll, M., Rief, N., Iwabuchi, K., Löbrich, M., and Jeggo, P.A. (2004). ATM and DNA-PK function redundantly to phosphorylate $\mathrm{H} 2 \mathrm{AX}$ after exposure to ionizing radiation. Cancer Res. 64, 2390-2396.

Stockdale, C., Flaus, A., Ferreira, H., and Owen-Hughes, T. (2006). Analysis of nucleosome repositioning by yeast ISWI and Chd1 chromatin remodeling complexes. J. Biol. Chem. 281, 16279-16288.

Stokes, D.G., and Perry, R.P. (1995). DNA-binding and chromatin localization properties of CHD1. Mol. Cell. Biol. 15, 2745-2753.

Stracker, T.H., and Petrini, J.H.J. (2011). The MRE11 complex: starting from the ends. Nat. Rev. Mol. Cell Biol. 12, 90-103.

Strahl, B.D., and Allis, C.D. (2000). The language of covalent histone modifications. Nature 403, 41-45.

Stuwe, T., Hothorn, M., Lejeune, E., Rybin, V., Bortfeld, M., Scheffzek, K., and Ladurner, A.G. (2008). The FACT Spt16 "peptidase" domain is a histone H3-H4 binding module. Proc. Natl. Acad. Sci. U. S. A. 105, 8884-8889.

Sugiyama, T., Zaitseva, E.M., and Kowalczykowski, S.C. (1997). A single-stranded DNAbinding protein is needed for efficient presynaptic complex formation by the Saccharomyces cerevisiae Rad51 protein. J. Biol. Chem. 272, 7940-7945.

Sullivan, K.D., Mullen, T.E., Marzluff, W.F., and Wagner, E.J. (2009). Knockdown of SLBP results in nuclear retention of histone mRNA. Rna New York N 15, 459-472.

Sun, Z.-W., and Allis, C.D. (2002). Ubiquitination of histone H2B regulates $\mathrm{H} 3$ methylation and gene silencing in yeast. Nature $418,104-108$.

Sun, Y., Jiang, X., Chen, S., Fernandes, N., and Price, B.D. (2005). A role for the Tip60 histone acetyltransferase in the acetylation and activation of ATM. Proc. Natl. Acad. Sci. U. S. A. $102,13182-13187$. 
Sun, Y., Jiang, X., and Price, B.D. (2010). Tip60: connecting chromatin to DNA damage signaling. Cell Cycle Georget. Tex 9, 930-936.

Sung, P., and Klein, H. (2006). Mechanism of homologous recombination: mediators and helicases take on regulatory functions. Nat. Rev. Mol. Cell Biol. 7, 739-750.

Symington, L.S. (2002). Role of RAD52 Epistasis Group Genes in Homologous Recombination and Double-Strand Break Repair. Microbiol. Mol. Biol. Rev. 66, 630-670.

Talbert, P.B., and Henikoff, S. (2010). Histone variants--ancient wrap artists of the epigenome. Nat. Rev. Mol. Cell Biol. 11, 264-275.

Tan, D., Marzluff, W.F., Dominski, Z., and Tong, L. (2013). Structure of histone mRNA stemloop, human stem-loop binding protein, and 3'hExo ternary complex. Science 339, 318-321.

Taylor, A.M.R., and Byrd, P.J. (2005). Molecular pathology of ataxia telangiectasia. J. Clin. Pathol. 58, 1009-1015.

Tharun, S., He, W., Mayes, A.E., Lennertz, P., Beggs, J.D., and Parker, R. (2000). Yeast Sm-like proteins function in mRNA decapping and decay. Nature 404, 515-518.

Thomas, J.O. (1999). Histone H1: location and role. Curr. Opin. Cell Biol. 11, 312-317.

Tibbetts, R.S., Brumbaugh, K.M., Williams, J.M., Sarkaria, J.N., Cliby, W.A., Shieh, S.-Y., Taya, Y., Prives, C., and Abraham, R.T. (1999). A role for ATR in the DNA damage-induced phosphorylation of p53. Genes Dev. 13, 152-157.

Tjeertes, J.V., Miller, K.M., and Jackson, S.P. (2009). Screen for DNA-damage-responsive histone modifications identifies H3K9Ac and H3K56Ac in human cells. Embo J. 28, 1878-1889.

Toh, Y., Pencil, S.D., and Nicolson, G.L. (1994). A novel candidate metastasis-associated gene, mta1, differentially expressed in highly metastatic mammary adenocarcinoma cell lines. cDNA cloning, expression, and protein analyses. J. Biol. Chem. 269, 2295822963.

Tsai, W.-B., Chung, Y.M., Takahashi, Y., Xu, Z., and Hu, M.C.-T. (2008). Functional interaction between FOXO3a and ATM regulates DNA damage response. Nat. Cell Biol. 10, 460467.

Tsukuda, T., Fleming, A.B., Nickoloff, J.A., and Osley, M.A. (2005). Chromatin remodelling at a DNA double-strand break site in Saccharomyces cerevisiae. Nature 438, 379-383.

Ulrich, H.D. (2011). Timing and spacing of ubiquitin-dependent DNA damage bypass. Febs Lett. 585, 2861-2867.

Uziel, T., Lerenthal, Y., Moyal, L., Andegeko, Y., Mittelman, L., and Shiloh, Y. (2003). Requirement of the MRN complex for ATM activation by DNA damage. Embo J. 22, 5612-5621. 
Vassilev, L.T., Vu, B.T., Graves, B., Carvajal, D., Podlaski, F., Filipovic, Z., Kong, N., Kammlott, U., Lukacs, C., Klein, C., et al. (2004). In vivo activation of the p53 pathway by smallmolecule antagonists of MDM2. Science 303, 844-848.

Vempati, R.K., Jayani, R.S., Notani, D., Sengupta, A., Galande, S., and Haldar, D. (2010). p300mediated acetylation of histone $\mathrm{H} 3$ lysine 56 functions in DNA damage response in mammals. J. Biol. Chem. 285, 28553-28564.

Walfridsson, J., Khorosjutina, O., Matikainen, P., Gustafsson, C.M., and Ekwall, K. (2007). A genome-wide role for CHD remodelling factors and Nap1 in nucleosome disassembly. Embo J. 26, 2868-2879.

Walker, A.I., Hunt, T., Jackson, R.J., and Anderson, C.W. (1985). Double-stranded DNA induces the phosphorylation of several proteins including the 90000 mol. wt. heatshock protein in animal cell extracts. Embo J. 4, 139-145.

Wang, B., Matsuoka, S., Carpenter, P.B., and Elledge, S.J. (2002). 53BP1, a mediator of the DNA damage checkpoint. Science 298, 1435-1438.

Wang, M., Wu, W., Wu, W., Rosidi, B., Zhang, L., Wang, H., and Iliakis, G. (2006). PARP-1 and $\mathrm{Ku}$ compete for repair of DNA double strand breaks by distinct NHEJ pathways. Nucleic Acids Res. 34, 6170-6182.

Wei, Y., Jin, J., and Harper, J.W. (2003). The cyclin E/Cdk2 substrate and Cajal body component p220(NPAT) activates histone transcription through a novel LisH-like domain. Mol. Cell. Biol. 23, 3669-3680.

Wells, D., and Kedes, L. (1985). Structure of a human histone cDNA: evidence that basally expressed histone genes have intervening sequences and encode polyadenylylated mRNAs. Proc. Natl. Acad. Sci. U. S. A. 82, 2834-2838.

West, S.C. (2003). Molecular views of recombination proteins and their control. Nat. Rev. Mol. Cell Biol. 4, 435-445.

Williams, R.S., Williams, J.S., and Tainer, J.A. (2007). Mre11-Rad50-Nbs1 is a keystone complex connecting DNA repair machinery, double-strand break signaling, and the chromatin template. Biochem. Cell Biol. Biochim. Biol. Cell. 85, 509-520.

Woodage, T., Basrai, M.A., Baxevanis, A.D., Hieter, P., and Collins, F.S. (1997). Characterization of the CHD family of proteins. Proc. Natl. Acad. Sci. U. S. A. 94, 11472-11477.

Wu, C.H., and Gall, J.G. (1993). U7 small nuclear RNA in C snurposomes of the Xenopus germinal vesicle. Proc. Natl. Acad. Sci. 90, 6257-6259.

Wu, R.S., and Bonner, W.M. (1981a). Separation of basal histone synthesis from S-phase histone synthesis in dividing cells. Cell 27, 321-330.

Wu, R.S., and Bonner, W.M. (1981b). Separation of basal histone synthesis from S-phase histone synthesis in dividing cells. Cell 27, 321-330. 
Wu, J., Chen, Y., Lu, L.-Y., Wu, Y., Paulsen, M.T., Ljungman, M., Ferguson, D.O., and Yu, X. (2011). Chfr and RNF8 synergistically regulate ATM activation. Nat. Struct. Mol. Biol. 18, 761-768.

Xiao, T., Kao, C.-F., Krogan, N.J., Sun, Z.-W., Greenblatt, J.F., Osley, M.A., and Strahl, B.D. (2005). Histone H2B ubiquitylation is associated with elongating RNA polymerase II. Mol. Cell. Biol. 25, 637-651.

Xie, A., Odate, S., Chandramouly, G., and Scully, R. (2010). H2AX post-translational modifications in the ionizing radiation response and homologous recombination. Cell Cycle Georget. Tex 9, 3602-3610.

Xu, Y., Sun, Y., Jiang, X., Ayrapetov, M.K., Moskwa, P., Yang, S., Weinstock, D.M., and Price, B.D. (2010). The p400 ATPase regulates nucleosome stability and chromatin ubiquitination during DNA repair. J. Cell Biol. 191, 31-43.

Xu, Y., Ayrapetov, M.K., Xu, C., Gursoy-Yuzugullu, O., Hu, Y., and Price, B.D. (2012). Histone H2A.Z controls a critical chromatin remodeling step required for DNA double-strand break repair. Mol. Cell 48, 723-733.

Yamane, K., Wu, X., and Chen, J. (2002). A DNA damage-regulated BRCT-containing protein, TopBP1, is required for cell survival. Mol. Cell. Biol. 22, 555-566.

Yan, B., Yang, X., Lee, T.-L., Friedman, J., Tang, J., Van Waes, C., and Chen, Z. (2007). Genome-wide identification of novel expression signatures reveal distinct patterns and prevalence of binding motifs for p53, nuclear factor-kappaB and other signal transcription factors in head and neck squamous cell carcinoma. Genome Biol. 8, R78.

Yarnell, A.T., Oh, S., Reinberg, D., and Lippard, S.J. (2001). Interaction of FACT, SSRP1, and the high mobility group (HMG) domain of SSRP1 with DNA damaged by the anticancer drug cisplatin. J. Biol. Chem. 276, 25736-25741.

Ye, X., Wei, Y., Nalepa, G., and Harper, J.W. (2003). The cyclin E/Cdk2 substrate p220(NPAT) is required for S-phase entry, histone gene expression, and Cajal body maintenance in human somatic cells. Mol. Cell. Biol. 23, 8586-8600.

You, Z., Bailis, J.M., Johnson, S.A., Dilworth, S.M., and Hunter, T. (2007). Rapid activation of ATM on DNA flanking double-strand breaks. Nat. Cell Biol. 9, 1311-1318.

Yu, D.S., and Cortez, D. (2011). A role for CDK9-cyclin K in maintaining genome integrity. Cell Cycle Georget. Tex 10, 28-32.

Yu, X., and Chen, J. (2004). DNA damage-induced cell cycle checkpoint control requires CtIP, a phosphorylation-dependent binding partner of BRCA1 C-terminal domains. Mol. Cell. Biol. 24, 9478-9486.

Yu, D.S., Zhao, R., Hsu, E.L., Cayer, J., Ye, F., Guo, Y., Shyr, Y., and Cortez, D. (2010). Cyclindependent kinase 9-cyclin $\mathrm{K}$ functions in the replication stress response. EMBO Rep. 11, 876-882. 
Yuan, J., Pu, M., Zhang, Z., and Lou, Z. (2009). Histone H3-K56 acetylation is important for genomic stability in mammals. Cell Cycle Georget. Tex 8, 1747-1753.

Zhang, F., and Yu, X. (2011). WAC, a functional partner of RNF20/40, regulates histone H2B ubiquitination and gene transcription. Mol. Cell 41, 384-397.

Zhao, H., Langerød, A., Ji, Y., Nowels, K.W., Nesland, J.M., Tibshirani, R., Bukholm, I.K., Kåresen, R., Botstein, D., Børresen-Dale, A.-L., et al. (2004). Different gene expression patterns in invasive lobular and ductal carcinomas of the breast. Mol. Biol. Cell 15, 2523-2536.

Zhao, J., Dynlacht, B., Imai, T., Hori, T., and Harlow, E. (1998). Expression of NPAT, a novel substrate of cyclin E-CDK2, promotes S-phase entry. Genes Dev. 12, 456-461.

Zhao, J., Kennedy, B.K., Lawrence, B.D., Barbie, D.A., Matera, A.G., Fletcher, J.A., and Harlow, E. (2000). NPAT links cyclin E-Cdk2 to the regulation of replicationdependent histone gene transcription. Genes Dev. 14, 2283-2297.

Zheng, L., Roeder, R.G., and Luo, Y. (2003). S phase activation of the histone H2B promoter by OCA-S, a coactivator complex that contains GAPDH as a key component. Cell 114, $255-266$.

Zhu, B., Zheng, Y., Pham, A.-D., Mandal, S.S., Erdjument-Bromage, H., Tempst, P., and Reinberg, D. (2005). Monoubiquitination of human histone H2B: the factors involved and their roles in HOX gene regulation. Mol. Cell 20, 601-611. 


\section{Acknowledgements}

First of all, I would like to express my sincere gratitude to my supervisor Prof. Dr. Steven A. Johnsen for giving me the opportunity to work with him. I am thankful for his excellent scientific supervision, his inspirational and encouraging ideas and all his help during my stay in Germany. Moreover, I want to thank him for the friendly atmosphere he created in our group and for being available at all times. I could not have imagined having a better supervisor for my Ph.D study.

I thank my thesis committee members Prof. Dr. Holger Reichardt and Prof. Dr. Ralph Kehlenbach for the inspiring discussions and their support throughout the thesis work. In addition, I appreciate Prof. Dr. Holger Reichardt for being the thesis second reviewer.

Many thanks go to Prof. Dr. Matthias Dobbelstein for giving me the opportunity to start my work in his department. I also want to thank all members of the Institute of Molecular Oncology for a nice and helpful working atmosphere. Further, I am thankful to Prof. Dr. Klaus Pantel and the Department of Tumor Biology for the very warm welcome after moving to Hamburg.

In particular, I would like to thank all the current and former members of the AG Johnsen for a wonderful working atmosphere and a great time spent together. Special thanks also go to my colleagues Magali, Sasha and Sanjay for their help in doing experiments.

I would like to thank Prof. Dr. Hans Will and Dr. Andreas Mund for great help, discussions and valuable advices in planning experiments. Also many thanks go to Dr. Wael Yassin Mansour for his help with the DNA repair experiments and suggestions.

Last but not the least, my deepest gratitude go to my parents, my sisters and my life partner Giri for their continuous support, constant encouragement, understanding and being there for me at every step of the way. 


\section{Curriculum Vitae}

\section{Vijayalakshmi Kari}

\section{Personal Details}

Date of birth

Nationality

Email

Telephone

Address

\section{Education}

July 2012 - till date

Jan 2010 to June 2012

Aug 2007 to Nov 2009

July 2005 to May 2007

June 2002 to Apr 2005

\author{
$10^{\text {th }}$ April 1985 \\ Indian \\ vijji15@gmail.com \\ 017634912973 \\ Wendloher weg 7, 20251, Hamburg
}

Ph.D. student at University Medical Center Hamburg-Eppendorf, Germany

Ph.D. student at University Medical Centre Göttingen, Germany Junior Research Fellow at Indian Institute of Science (IISC), India Master of Science in Biochemistry and Molecular Biology, Pondicherry Central University, India

Bachelor of Science, Nagarjuna University, India

\section{Research Experience}

July 2012 - until present - Ph.D student

Department of Tumor Biology University Medical Center Hamburg-Eppendorf, Germany Supervisor: Prof. Dr. Steven A. Johnsen

Role of Histone Metabolism and Chromatin Structure in DNA repair

Jan 2010 to June 2012- Ph.D student

Institute of Molecular Oncology, University Medical Centre Göttingen, Germany Supervisor: Prof. Dr. Steven A. Johnsen

Role of Histone Metabolism and Chromatin Structure in DNA repair

\section{Aug 2007 to Nov 2009 - Junior research fellow}

Department of Biochemistry, Indian Institute of Science (IISC), Bangalore, India

Supervisor: Prof. Dr. Sathees C Raghavan

Role of BCL2 in non-homologous end joining pathway 


\section{Dec 2004 to Apr 2005 - Master thesis}

Department of Biochemistry and Molecular Biology, Pondicherry University, India

Supervisor: Dr. Suresh Yenugu

Expression of Toll Like Receptors in rat male reproductive system

\section{May 2004 to June 2004 - Rotation Student}

School of Studies in Neuroscience, Jiwaji University, Gwalior, India

Supervisor: Prof. Ishan Patro

\section{Publications}

1. Kari V*, Karpiuk O*, Tieg B, Kriegs M, Dikomey E, Krebber H, Begus-Nahrmann Y, Johnsen $\mathrm{SA}$. A subset of histone $\mathrm{H} 2 \mathrm{~B}$ genes produces polyadenylated mRNAs under a variety of cellular conditions. PLoS ONE 8(5): e63745.doi:10.1371/journal.pone.0063745. * Equal authorship.

2. Kari $V$, Shchebet A, Neumann H, Johnsen SA. The H2B ubiquitin ligase RNF40 cooperates with SUPT16H to induce dynamic changes in chromatin structure during DNA double-strand break repair. Cell Cycle. 2011 Oct 15;10(20):3495-504

3. Kumar TS*, Kari V*, Choudhary B, Nambiar M, Akila TS, Raghavan SC. Anti-apoptotic protein BCL2 down-regulates DNA end joining in cancer cells. J Biol Chem. 2010 Oct 15;285(42):32657-70. * Equal authorship

4. Chiruvella KK, Kari V, Choudhary B, Nambiar M, Ghanta RG, Raghavan SC. FEBS Lett. Methyl angolensate, a natural tetranortriterpenoid induces intrinsic apoptotic pathway in leukemic cells. 2008 Dec 10;582(29):4066-76.

5. Nambiar M, Kari V, Raghavan SC. Chromosomal translocations in cancer. Biochim Biophys Acta. 2008 Dec;1786(2):139-52. Review.

\section{References}

Prof. Dr. Steven A. Johnsen

Department Tumor Biology

University Medical Center Hamburg-Eppendorf, Germany

Email: steven.a.johnsen@gmail.de

+49 (0)40 7410-57495

Prof. Sathees C Raghavan

Department of Biochemistry

Indian Institute of Science, Bangalore, India

Email: sathees@biochem.iisc.ernet.in

+91(0)8022932674 